University of Nebraska - Lincoln

DigitalCommons@University of Nebraska - Lincoln

\title{
Postglacial Inflation-Deflation Cycles, Tilting, and Faulting in the Yellowstone Caldera Based on Yellowstone Lake Shorelines
}

\author{
Kenneth L. Pierce \\ U.S. Geological Survey, kpierce@usgs.gov \\ Kenneth P. Cannon \\ U.S. Geological Survey \\ Grant A. Meyer \\ U.S. Geological Survey \\ Matthew J. Trebesch \\ U.S. Geological Survey \\ Raymond D. Watts \\ U.S. Geological Survey
}

Follow this and additional works at: https://digitalcommons.unl.edu/usgspubs

Part of the Earth Sciences Commons

Pierce, Kenneth L.; Cannon, Kenneth P.; Meyer, Grant A.; Trebesch, Matthew J.; and Watts, Raymond D., "Postglacial Inflation-Deflation Cycles, Tilting, and Faulting in the Yellowstone Caldera Based on Yellowstone Lake Shorelines" (2007). Publications of the US Geological Survey. 78.

https://digitalcommons.unl.edu/usgspubs/78

This Article is brought to you for free and open access by the US Geological Survey at DigitalCommons@University of Nebraska - Lincoln. It has been accepted for inclusion in Publications of the US Geological Survey by an authorized administrator of DigitalCommons@University of Nebraska - Lincoln. 


\section{Postglacial Inflation-Deflation Cycles, Tilting, and Faulting in the Yellowstone Caldera Based on Yellowstone Lake Shorelines}

By Kenneth L. Pierce, Kenneth P. Cannon, Grant A. Meyer, Matthew J. Trebesch, and Raymond D. Watts

Chapter $\mathrm{E}$ of

Integrated Geoscience Studies in the Greater Yellowstone AreaVolcanic, Tectonic, and Hydrothermal Processes in the Yellowstone Geoecosystem

Edited by Lisa A. Morgan

U.S. Geological Survey Professional Paper 1717 


\section{Contents}

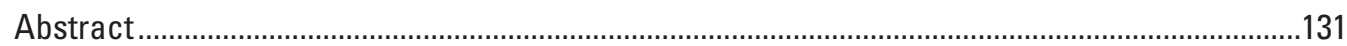

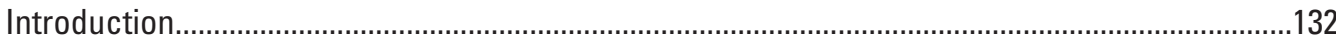

Previous Studies of Postglacial Shorelines ....................................................................................132

Holocene Lake- and River-Level Changes and Their Chronology ..................................................135

Lake-Level Rise After Lake Low 4-3 ka ...............................................................................143

Yellowstone Lake ..........................................................................................................143

Outlet Reach of the Yellowstone River ......................................................................... 143

S-Meander and Rise of Lake $9.7-8.6$ ka.................................................................................145

Subaerial Shoreline Sequence $~ 8.0$ ka S2 to 14.4 ka S6 ......................................................146

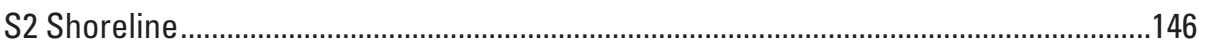

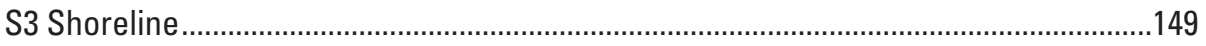

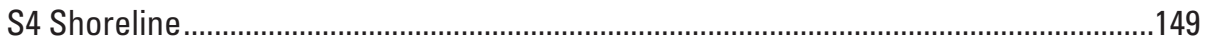

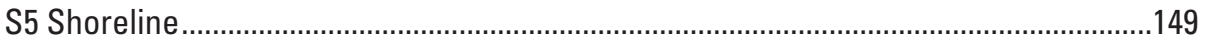

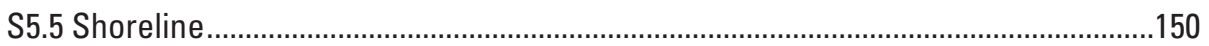

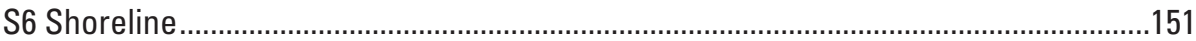

Possible Lake-Level Culminations Near 4.5 and 2.5 ka ........................................................151

Summary of Postglacial Lake-Level Chronology.....................................................................152

Uplift of Le Hardys Rapids Indicated by Submerged Localities ..........................................153

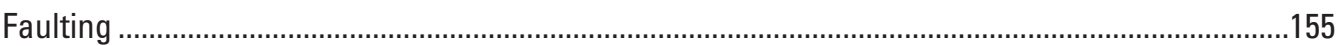

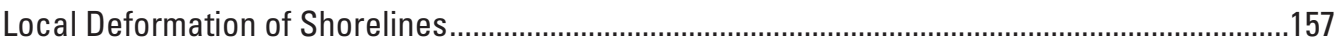

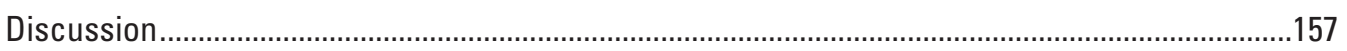

Decreases in Lake Level...................................................................................................... 158

Holocene Outlet Erosion and Pleistocene Glacial Damming .........................................158

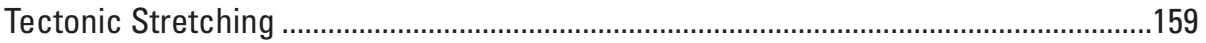

Yellowstone Lake Ceasing to Overflow .................................................................... 159

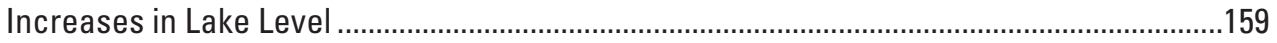

Inflation by Magma Intrusion........................................................................................ 159

Tectonic Compression....................................................................................................160

Possible Increases or Decreases in Lake Level......................................................................160

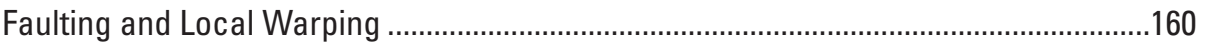

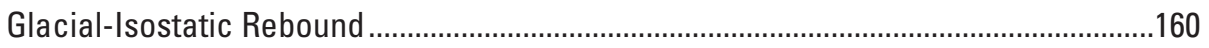

Oscillations in Lake Level .........................................................................................................160

Two Favored Explanations for Record of Oscillations ............................................................162

Hydrothermal Inflation and Deflation .............................................................................162

Inflation by Magma Intrusion and Subsidence by Other Mechanisms ..........................162

Local Doming and Faulting of Shorelines ...........................................................................163

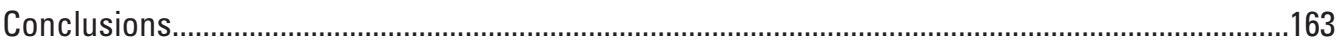

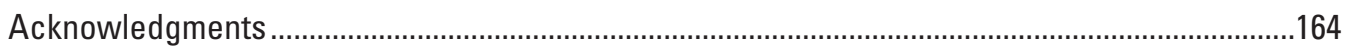

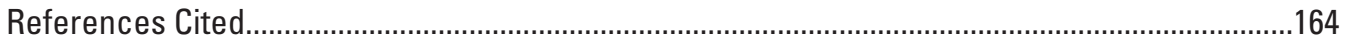




\section{Figures}

1. Map showing Yellowstone National Park, the Yellowstone caldera, Yellowstone Lake and River, and contours on the historic dome of uplift from1923-1975.

2. Map of the Yellowstone Lake area, including some important localities and ages outside the LIDAR area

3. Vertical surface displacements measured by repeated first-order leveling surveys along a traverse from Lake Butte north-northwestward across the floor of Yellowstone caldera to Canyon Junction....................................................135

4. Reconstruction of changes in Yellowstone Lake level during the last 15 k.y................136

5. LIDAR image showing the "outlet reach" of the Yellowstone River from the outlet to Le Hardys Rapids ............................................................................144

6. Diagram of uplift of Le Hardys Rapids after 3 ka ......................................................145

7. Profile section of the $\mathrm{S}$-meander based on LIDAR data ...............................................146

8. Cross section across the S-meander showing location of channel gravels, $\sim 9.2-\mathrm{ka}$ charcoal, and S3 and S2 shorelines

9. Comparison of outlet reach during $\mathrm{S}$-meander time ( $9.7 \mathrm{ka})$ with that during formation of $\mathrm{S} 3 / \mathrm{S} 2$ shorelines $(8.6-8.0 \mathrm{ka})$.

10. LIDAR image of the northern lakeshore area showing shorelines $S 2-S 6$ as well as unlabeled intermediate shorelines.

11. Drawings of projectile points from shorelines on the north shore of Yellowstone Lake.

12-15. LIDAR profiles:

12. Of northern Yellowstone Lake shorelines S2-S6

13. Along S5.5 barrier beach mantled by Mary Bay hydrothermal-explosion deposit

14. Showing faulting and tilting of shorelines across the Fishing Bridge peninsula

15. Of S4, S2 and two younger shorelines (S1.8 and S1.6) from the Storm Point geothermal center west to Pelican Creek.

16. Diagrams showing mechanisms for inflation and deflation of Yellowstone caldera 158

\section{Tables}

1. ${ }^{14} \mathrm{C}$ and other ages associated with levels of Yellowstone Lake

2. Submerged Yellowstone Lake and River levels, and their normalization to the outlet

3. Offsets on the Fishing Bridge fault and associated tilting across Fishing Bridge .........154

4. Processes for decreases, increases, and oscillations in Yellowstone Lake and River levels in postglacial time

5. Change in altitude of shorelines $\mathrm{S} 2, \mathrm{~S} 4$, and $\mathrm{S} 5$ 


\title{
Postglacial Inflation-Deflation Cycles, Tilting, and Faulting in the Yellowstone Caldera Based on Yellowstone Lake Shorelines
}

\author{
By Kenneth L. Pierce, ${ }^{1}$ Kenneth P. Cannon, ${ }^{2}$ Grant A. Meyer, ${ }^{3}$ Matthew J. Trebesch, ${ }^{1}$ and Raymond D. Watts ${ }^{4}$
}

\section{Abstract}

The Yellowstone caldera, like many other late Quaternary calderas of the world, exhibits dramatic unrest. Between 1923 and 1985, the center of the Yellowstone caldera rose nearly 1 $\mathrm{m}$ along an axis between its two resurgent domes (Pelton and Smith, 1979; Dzurisin and Yamashita, 1987). From 1985 until 1995-1996, the caldera subsided at about $2 \mathrm{~cm} / \mathrm{yr}$ (Dzurisin and others, 1990). More recent radar-interferometry studies show renewed inflation of the northeastern resurgent dome between 1995 and 1996; this inflation migrated to the southwestern resurgent dome from 1996 to 1997 (Wicks and others, 1998).

We extend this record back in time using dated geomorphic evidence of postglacial Yellowstone Lake shorelines around the northern shore and Yellowstone River levels in the outlet area. We date these shorelines using carbon-isotopic and archeological methods. Following Meyer and Locke (1986) and Locke and Meyer (1994), we identify the modern shoreline as S1 (1.9 \pm 0.3 $\mathrm{m}$ above the lake-gage datum), map paleoshoreline terraces $\mathrm{S} 2$ to S6, and infer that the prominent shorelines were cut during intracaldera-uplift episodes that produced rising water levels. Doming along the caldera axis reduces the gradient of the Yellowstone River from Le Hardys Rapids to the Yellowstone Lake outlet and ultimately causes an increase in lake level. The 1923-1985 doming is part of a longer uplift episode that has reduced the Yellowstone River gradient to a "pool" with a drop of only $0.25 \mathrm{~m}$ over most of this $5-\mathrm{km}$ reach. We also present new evidence that doming has caused submergence of some Holocene lake and river levels.

Shoreline S5 is about $14 \mathrm{~m}$ above datum and estimated to be $~ 12.6$ ka because it postdates a large hydrothermal-explosion deposit from the Mary Bay area that occurred $\sim 13 \mathrm{ka}$. S4 formed about $8 \mathrm{~m}$ above datum $\sim 10.7 \mathrm{ka}$ as dated by archeology and

${ }^{1}$ U.S. Geological Survey, Northern Rocky Mountain Science Center, Box 173492, Montana State University, Bozeman, MT 59717.

${ }^{2}$ National Park Service, Midwest Archeological Center, Federal Building, Lincoln, NE 68508-3873.

${ }^{3}$ Department of Earth and Planetary Sciences, University of New Mexico, Albuquerque, NM 87131-1116.

${ }^{4}$ U.S. Geological Survey, 4512 McMurry Ave., Fort Collins, CO 80525-3400.
${ }^{14} \mathrm{C}$ and was accompanied by offset on the Fishing Bridge fault. About $9.7 \mathrm{ka}$, the Yellowstone River eroded the "S-meander," followed by a $\sim 5-\mathrm{m}$ rise in lake level to $\mathrm{S} 2$. The lowest generally recognizable shoreline is $\mathrm{S} 2$; it is $\sim 5 \mathrm{~m}$ above datum ( $3 \mathrm{~m}$ above $\mathrm{S} 1$ ) and is $\sim 8 \mathrm{ka}$, as dated on both sides of the outlet. Yellowstone Lake and the Yellowstone River near Fishing Bridge were 5-6 $\mathrm{m}$ below their present level about 4-3 ka, as indicated by ${ }^{14} \mathrm{C}$ ages from submerged beach deposits, drowned valleys, and submerged Yellowstone River gravels. Thus, the lake in the outlet region has been below or near its present level for about half the time since a 1-km-thick ice cap melted from the Yellowstone Lake basin about $16 \mathrm{ka}$.

The amplitude of two rises in lake and river level can be estimated based on the altitude of Le Hardys Rapids, indicators of former lake and river levels, and reconstruction of the river gradient from the outlet to Le Hardys Rapids. Both between $\sim 9.5 \mathrm{ka}$ and $\sim 8.5 \mathrm{ka}$, and after $\sim 3 \mathrm{ka}$, Le Hardys Rapids was uplifted about $8 \mathrm{~m}$ above the outlet at Fishing Bridge, suggesting a cyclic deformation process. Older rises in lake level are suggested by locations where the $\sim 10.7-\mathrm{ka}$ S4 truncates older shorelines and where valleys were truncated by the $\sim 12.6-\mathrm{ka}$ S5 shoreline. Using these controls, a plot of lake level through time shows five to seven millennial-scale oscillations since $14.4 \mathrm{ka}$.

Major cycles of inflation and deflation are thousands of years long. Le Hardys Rapids has twice been uplifted $\sim 8 \mathrm{~m}$ relative to the lake outlet. These two locations span only the central 25 percent of the historic caldera doming; so if we use historic doming as a model, total projected uplift would be $\sim 32 \mathrm{~m}$. This "heavy breathing" of the central part of the Yellowstone caldera may reflect a combination of several possible processes: magmatic inflation; tectonic stretching and deflation; and hydrothermal-fluid sealing and inflation, followed by cracking of the seal, pressure release, and deflation. Over the entire postglacial period, subsidence has balanced or slightly exceeded uplift as shown by older shorelines that descend toward the caldera axis. We favor a hydrothermal mechanism for inflation and deflation because it provides for both inflation and deflation with little overall change. Other mechanisms, such as inflation by magma intrusion and deflation by extensional stretching, require two separate geologic processes to alternate and yet result in no net elevation change. 
In addition to inflation and deflation, new LIDAR (Light Detection and Ranging) data demonstrate previously unrecognized local deformation along the north shore of Yellowstone Lake. The newly recognized Fishing Bridge fault shows a progressive increase in offset from $0.5 \mathrm{~m}$ for the $\sim 8$-ka S2 to perhaps $5 \mathrm{~m}$ for the $~ 12.6-\mathrm{ka}$ S5. Uplift of the Storm Point hydrothermal center tilts shorelines westward as much as $6 \mathrm{~m} / \mathrm{km}$. A local anticline has as much as $3 \mathrm{~m}$ of relief in $0.5 \mathrm{~km}$. LIDAR data also show the Mary Bay hydrothermal-explosion debris has a surface relief of about $1 \mathrm{~m}$ over $100 \mathrm{~m}$, and that it overlies S5.5 and S6 shorelines, but not S5. Although the postglacial deformation record does not indicate voluminous magma accumulation or other large-scale eruption precursors, strong local deformation associated with hydrothermal centers does suggest the possibility of future hydrothermal explosions and associated hazards.

\section{Introduction}

The Yellowstone caldera (fig. 1) is the youngest of three large rhyolitic calderas formed in greater Yellowstone in the last 2.1 m.y. (Christiansen, 1984, 2001). It collapsed with eruption of $\sim 1,000 \mathrm{~km}^{3}$ of ash flows from two overlapping ring-fracture systems at $\sim 640 \mathrm{ka}$. Shortly thereafter, the central part of the caldera was uplifted to form the Sour Creek (northeast) and Mallard Lake (southwest) resurgent domes. Voluminous extrusion of rhyolitic lavas from $150 \mathrm{ka}$ to $70 \mathrm{ka}$ covered much of the caldera except the Sour Creek dome and small areas in the eastern part of Yellowstone Lake (Morgan and Shanks, 2005)

(fig. 2). At present, this caldera shows remarkable signs of unrest, consistent with many other young calderas around the world (Newhall and Dzurisin, 1988; Dvorak and Mastrolorenzo, 1991). A resurvey of 1923 level lines in 1975-1977 along the road system of Yellowstone showed doming within the caldera (fig. 1), with maximum uplift at Le Hardys Rapids (LHR) of about $0.8 \mathrm{~m}$ (Pelton and Smith, 1979). Between 1976 and 1985, resurveys revealed an additional 0.15-m uplift of LHR, with a pattern similar to that between 1923 and 1976 (Dzurisin and Yamashita, 1987). Thus, from 1923 to 1985, maximum uplift was about $0.95 \mathrm{~m}$ over 62 years at an average rate of about 15 mm/yr. Uplift unexpectedly ceased in 1985, and between 1986 and 1996, the caldera subsided at a maximum rate of about $20 \mathrm{~mm} / \mathrm{yr}$ in a pattern essentially inverse to the uplift (fig. 3). Recent radar-interferometry studies define a more complex temporal pattern, with renewed inflation of the Sour Creek resurgent dome area from 1995 to 1996 and migration of inflation to the Mallard Lake dome area from 1996 to 1997 (Wicks and others, 1998). Global positioning system (GPS) measurements from 1987 to 1995 (Smith and others, 1997; Meertens and others, 2000) show caldera-wide subsidence

of as much as $15 \mathrm{~mm} / \mathrm{yr}$ accompanied by radial-caldera contraction of up to $10 \mathrm{~mm} / \mathrm{yr}$.

Spurred by these records of uplift and subsidence in the Yellowstone caldera over the last century, we employed geomorphic criteria to assess the character and magnitude of deformation during the last 15,000 years. Paleoshorelines of Yellowstone Lake and drowned subaerial features, such as stream valleys and beach sediment, allow us to construct a record of vertical deformation in the central part of the Yellowstone caldera. New ${ }^{14} \mathrm{C}$ and projectile-point ages obtained in archeological surveys and excavations, as well as geological studies, provide improved age control (Pierce and others, 1994; Cannon and others, 1994, 1995, 1997). Cyclic inflation and deflation of the caldera in the last 100 years has been called "breathing" (see, for example, Pelton and Smith, 1979); we use the term "heavy breathing" for longer, higher amplitude cycles of inflation and deflation.

We build this study on the detailed surveying of Yellowstone Lake shorelines by Meyer and Locke (1986), Locke (1986), Meyer (1986), and Locke and Meyer (1994). Our findings are in general agreement with their mapping and correlation of shorelines; however, new dating and archeological studies indicate that the lower shorelines are much older than previously thought. LIDAR imagery of the northern lakeshore permits more continuous tracing and elevation measurement of shorelines and revision of some shoreline correlations east of Pelican Creek. LIDAR imagery also allowed us to locate previously unrecognized features, including the Fishing Bridge fault and low-relief beach-ridge shorelines that are strongly tilted away from the Storm Point geothermal center. Because of delays in publication, Pierce and others (2002) made available an earlier version of this study.

\section{Previous Studies of Postglacial Shorelines}

Richmond first mapped shorelines from $3 \mathrm{~m}$ to more than $50 \mathrm{~m}$ above present Yellowstone Lake (Richmond, 1973, 1974, 1976, 1977; Richmond and Pierce, 1972). He considered shorelines at and below 18-20 m to represent an open, postglacial lake, whereas higher shorelines were associated with deglaciation of the lake basin. Richmond's work predated recognition of active deformation in the Yellowstone caldera, and he assumed that (1) shorelines could be correlated by height alone, and (2) lake level underwent a relatively simple and continuous decline over postglacial time.

Drawing on this earlier work, Meyer and Locke (1986) mapped and correlated about nine shorelines around northern Yellowstone Lake. They designated the present shoreline as $\mathrm{S} 1$ and shorelines up to about $30 \mathrm{~m}$ above $\mathrm{S} 1$ as $\mathrm{S} 2$ to S9. Shoreline profiles were surveyed to centimeter accuracy (with closure) using the Bridge Bay lake-level gage zero mark as a datum, and shoreline elevations were interpreted to the decimeter. Meyer and Locke (1986) inferred that prominent shorelines were cut by rising lake levels due to episodic uplift within the Yellowstone caldera. This work was later continued around the entire lake (Locke and Meyer, 1994). In contrast to earlier work that relied on rapid hand leveling, these studies showed that many shorelines were deformed, both inside and 


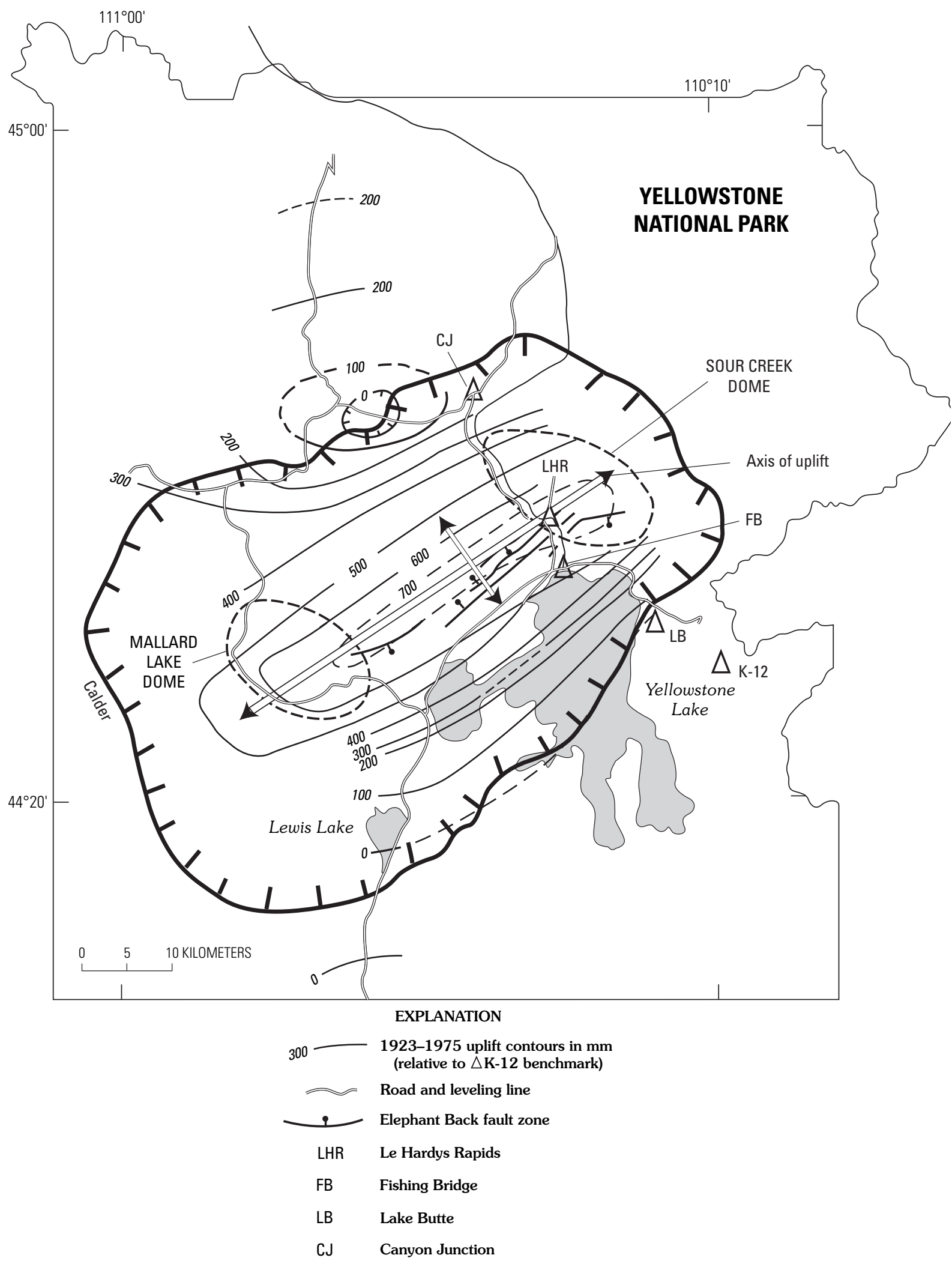

Figure 1. Map showing Yellowstone National Park, the Yellowstone caldera, Yellowstone Lake and Yellowstone River, and contours on the historic dome of uplift from 1923-1975 (after Pelton and Smith, 1982). Uplift is primarily within the Yellowstone caldera, and the axis of uplift extends between the Sour Creek and Mallard Lake domes. Note that upstream from Le Hardys Rapids (LHR) uplift also raises the level of the Yellowstone River and Yellowstone Lake and thus ties the level of Yellowstone Lake to uplift and subsidence at LHR. 


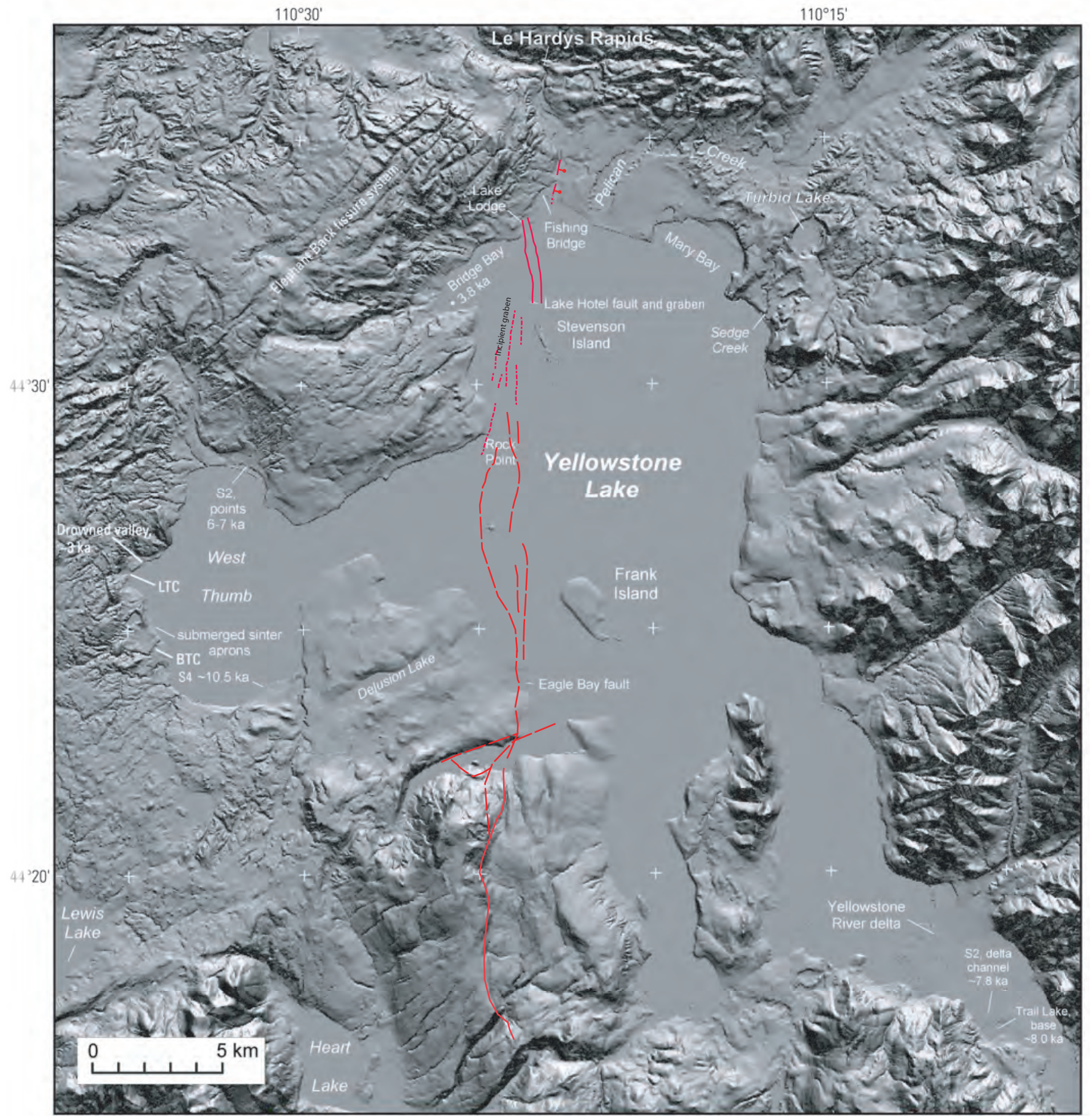

Figure 2. Digital elevation model of the Yellowstone Lake area, including some important localities and ages outside the LIDAR area. LIDAR data (figs. 5 and 10) cover the north shore from near the Lake Lodge to Mary Bay. Lake-floor surveys (Morgan and others, this volume) suggest a zone of faulting and fissuring connecting the Holocene Eagle Bay fault (Locke and others, 1992) in the southern lake area with the Lake Hotel graben. BTC, Big Thumb Creek; LTC, Little Thumb Creek. 


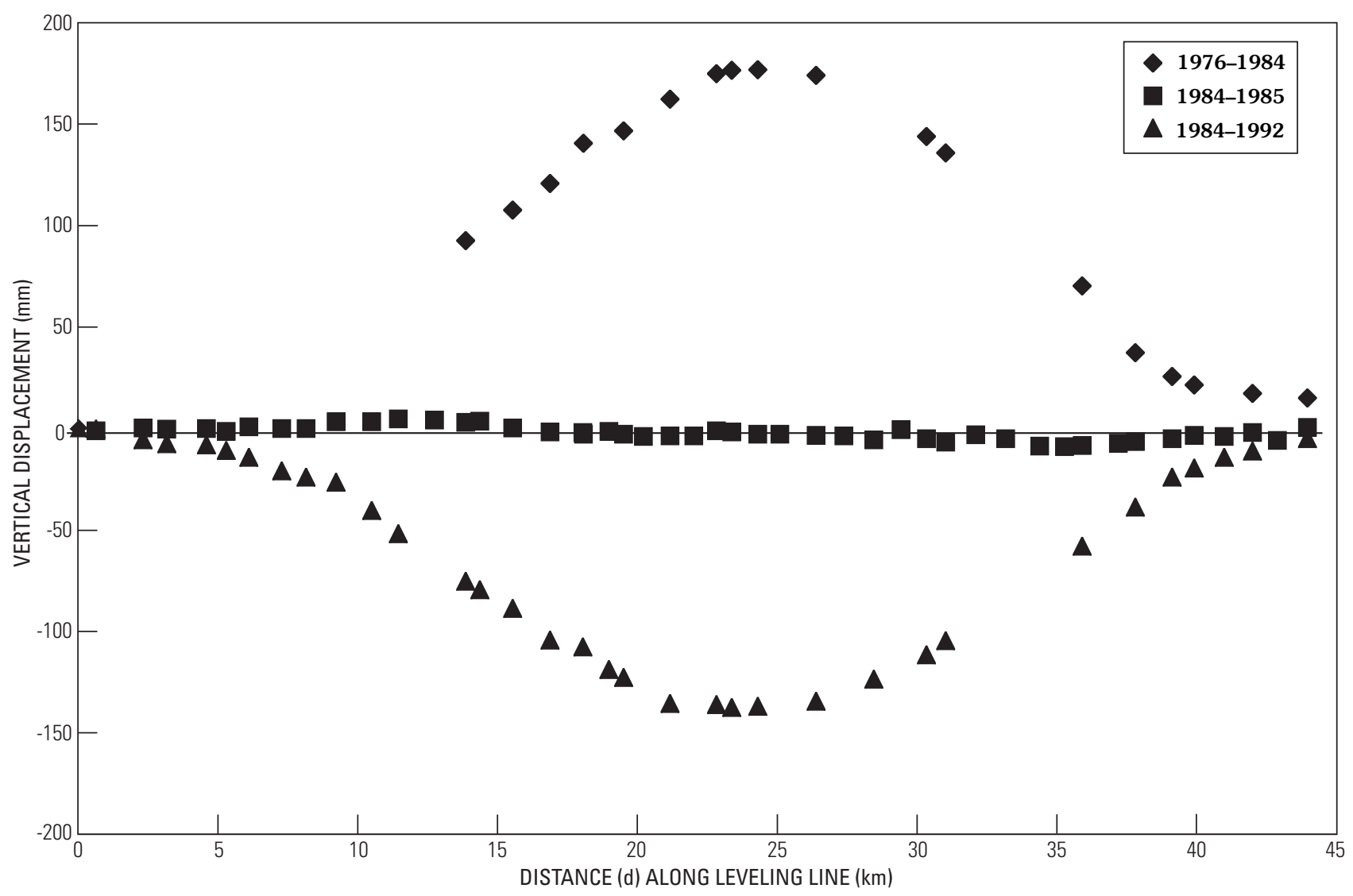

Figure 3. Vertical surface displacements measured by repeated first-order leveling surveys along a traverse from Lake Butte $(d=0)$ north-northwest across the floor of Yellowstone caldera to Canyon Junction $(d=44 \mathrm{~km})$. The traverse was measured in 1923, 1975-1977 (labeled 1976 in the plot), each year from 1983 to 1993, 1995, and 1998. Shown here are the net displacements of benchmarks along the traverse for three contiguous time periods: 1976-1984, 1984-1985, and 1984-1992 (after Dzurisin and others, 1994). The uplift profile for 1976-1984 is essentially the mirror image of the subsidence profile for 1984-1992, which suggests a common source region and a remarkable unity of the uplift and subsidence processes. The transition from uplift to subsidence occurred during 1984-1985 when the observed surface displacements were negligibly small.

outside the caldera. Except for local areas of greater tilting and faulting, most shorelines were found to be gently warped. Given that historic intracaldera uplift and subsidence rates of $10-20 \mathrm{~mm} / \mathrm{yr}$ could produce $50-100 \mathrm{~m}$ of vertical deformation in 5,000 yr, Locke and Meyer (1994) noted that net intracaldera deformation during the Holocene must be small because most shorelines are subhorizontal and the highest, oldest postglacial shorelines are at similar elevations inside and outside of the caldera $( \pm \sim 8 \mathrm{~m})$. In these studies, a small number of radiocarbon dates were obtained on organic matter in lagoonal sediments associated with shorelines, but these provided only minimum ages.

During archeological studies near the outlet of Yellowstone Lake, Reeve (1989) initially recognized a discrepancy between Richmond's declining lake model and the occurrence of late Pleistocene-early Holocene (paleo-indian) projectile points within a few meters of present lake level, but he did not note that his archeological studies required considerably older ages than the minimum-limiting dates of Meyer and Locke (1986). Hamilton and Bailey (1990) recognized submerged shorelines of Yellowstone Lake at depths of 3-30 m below the present lake surface and constructed a complex history of postglacial lake-level changes but with little age control or means of correlation. Submerged shorelines are also described by Johnson and others (2003).

\section{Holocene Lake- and River-Level Changes and Their Chronology}

In this section we present the data used to construct the detailed lake-level chronology of figure 4 . Table 1 describes the radiocarbon sample locations and gives the calibrated (corrected) ages. We use "ka" to indicate thousand years ago in calibrated (corrected) ages. Elevation is relative to the Bridge Bay gage. We first present evidence for low lake and river levels that were followed by a rise in lake and river levels because this is the key observation indicating caldera inflation. We then describe 


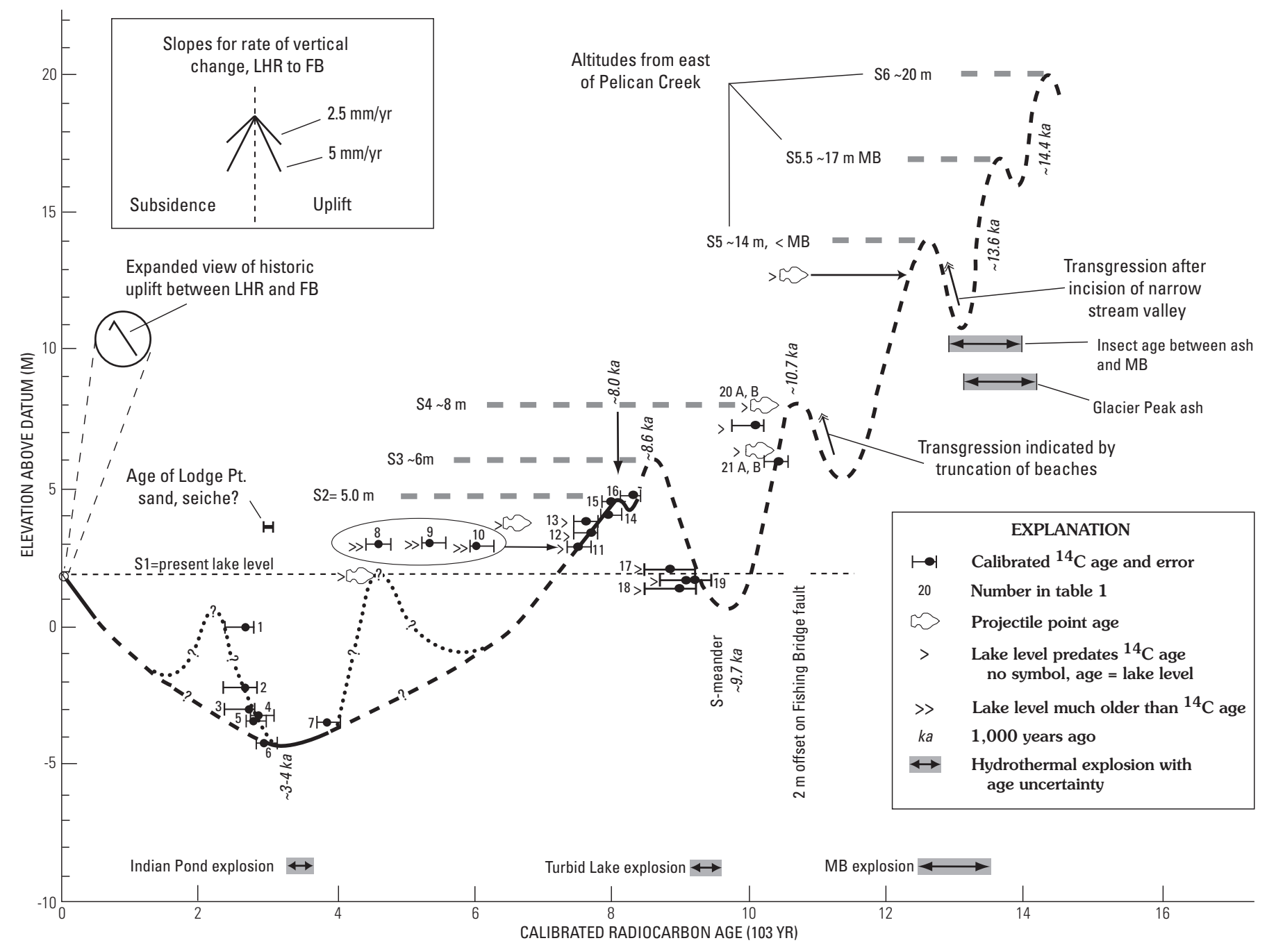

Figure 4. Reconstruction of changes in Yellowstone Lake level during the last 15 k.y. Shoreline altitude is relative to gage at Bridge Bay Marina and for the northern lake area. Radiocarbon ages and their conversion to calendar ages are given in table 1. LHR, Le Hardys Rapids; FB, Fishing Bridge; MB, Mary Bay explosion.

subaerial shorelines in order of increasing age because this is how we resolved the chronology shown in figure 4.

Airborne LIDAR elevation data were incorporated into this study. The survey contractor, Eaglescan, Inc., flew its instrumented airplane approximately $1,500 \mathrm{~m}$ above ground level. Aircraft position was recorded using base-stationcorrected global positioning system (GPS) measurements, and aircraft attitude was measured with an inertial navigation system (INS). The laser was in the near-infrared wavelength (1.069 micron) and recorded returns from the land and water surface, reflecting these from a cross-track scanning mirror. The same laser that generated the transmissions amplified ground-return pulses for detection and measurement of the round-trip time of light. Recording equipment was configured to log the last return pulse, thus discarding a large fraction of vegetation signals. Each LIDAR pulse was approximately 15 $\mathrm{cm}$ thick along its flight path (this is the instrumental resolution limit for elevation determinations) and approximately $1 \mathrm{~m}$ in diameter at its intersection with the ground.

The contractor's postflight data-processing combined GPS positions, INS attitude measurements, mirror positions, and laser-pulse time-of-flight measurements to determine the georeferenced location of each reflection point in UTM coordinates (Zone 12, WGS84 reference system). The scanning mirror operated within $15^{\circ}$ of nadir, measuring a swath width of $800 \mathrm{~m}$ along each flight line. Geometric factors degrade the accuracy of position determinations at points farther from nadir; errors at $15^{\circ}$ are approximately $1 \mathrm{~m}$. The laser-pulserepetition frequency and mirror-scan rate were sufficient to sample elevations at a nominal 2-m spacing across-track and 5-m spacing along-track.

Postflight processing included winnowing of returns that came from vegetation and other features above the mean neighborhood surface. The remaining returns, presumed to come from the ground or from low ground-cover vegetation, 
Table 1. Carbon-14 and other ages associated with levels of Yellowstone Lake, generally in order of increasing age.

[Localities and ages in bold type are critical to interpretation of lake-level history. YR, Yellowstone River; FB, Fishing Bridge]

\begin{tabular}{|c|c|c|c|c|}
\hline $\begin{array}{l}\text { Location, sample identifier, depth } \\
\text { Samples numbered } 1 \text { to } 23 \text { are } \\
\text { plotted in fig. } 4\end{array}$ & $\begin{array}{l}\text { Age (yr B.P.) } \\
\text { Lab number(s) }\end{array}$ & $\begin{array}{l}\text { Corrected age (yr) } \\
\text { (2-sigma range) Method A }\end{array}$ & $\begin{array}{l}\text { Meters above } \\
\text { datum }\end{array}$ & Remarks \\
\hline \multicolumn{5}{|c|}{ A. Subaerial samples lower and younger than S-2 shoreline } \\
\hline $\begin{array}{l}\text { 1. Pelican Cr. Paleo-barrier } \\
\text { beach }\end{array}$ & $\begin{array}{c}\mathbf{2 , 5 5 0} \pm \mathbf{6 0} \\
\text { Beta- }-78912\end{array}$ & $\begin{array}{c}2,735 \\
(2,362-2,775)\end{array}$ & $0-1$ & $\begin{array}{l}\text { From depth of } 1.65 \mathrm{~m} \text { in eolian sand and } 0.25 \mathrm{~m} \text { above top of openwork beach } \\
\text { gravel near present Yellowstone Lake level. }\end{array}$ \\
\hline $94 \mathrm{P} 33 \mathrm{~b}, 165 \mathrm{~cm}$ & CAMS-17814 & & & \\
\hline $\begin{array}{l}\text { Pelican Cr. East reentrant } \\
\text { 97P29, } 81 \mathrm{~cm}\end{array}$ & $\begin{array}{c}2,800 \pm 50 \\
\text { WW- }-1635\end{array}$ & $\begin{array}{c}2,874-2,917 \\
(2,778-3,057)\end{array}$ & 4.5 & $\begin{array}{l}\text { Charcoal from mixed zone on } 4.5 \mathrm{~m} \text { beach, provides minimum age for post-S2 } \\
\text { beach. }\end{array}$ \\
\hline 97P30, $65 \mathrm{~cm}$ & $\begin{array}{c}2,670 \pm 50 \\
W W-1636\end{array}$ & $\begin{array}{c}2,770 \\
(2,739-2,865)\end{array}$ & 4.2 & $\begin{array}{l}\text { Charcoal from mixed zone on } 4.2 \mathrm{~m} \text { beach, provides minimum age for post-S2 } \\
\text { beach. }\end{array}$ \\
\hline $97 \mathrm{P} 31,65 \mathrm{~cm}$ & Modern & & ? & Pine needles, brown. \\
\hline $\begin{array}{l}\text { Storm Point } \\
95 \mathrm{P} 61,90 \mathrm{~cm}\end{array}$ & $\begin{array}{c}\mathbf{2 , 1 6 0 \pm 6 0} \\
\text { WW-724 } \\
\text { CAMS-28372 }\end{array}$ & $\begin{array}{c}2,133-2,148 \\
(1,954-2,335)\end{array}$ & 3.43 & $\begin{array}{l}\text { Charcoal from open platy platform gravels of shoreline } 4.3 \mathrm{~m} \text { above datum on } \\
\text { east side of Storm Point. Anomalously young age probably explained by uplift of } \\
\text { Storm Point geothermal center. }\end{array}$ \\
\hline $95 \mathrm{P} 61,67 \mathrm{~cm}$ & $\begin{array}{c}1,160 \pm 40 \\
\text { WW-723 } \\
\text { CAMS-28371 }\end{array}$ & $\begin{array}{c}1,060 \\
(968-1,174)\end{array}$ & 3.62 & $\begin{array}{l}\text { Charcoal from eolian deposits overlying the above sample. May be local young } \\
\text { uplift of Storm Point. }\end{array}$ \\
\hline \multicolumn{5}{|c|}{ B. Samples from below present lake level } \\
\hline $\begin{array}{l}\text { 2. Drowned YR channel } \\
91 \mathrm{P} 46,280 \mathrm{~cm}\end{array}$ & $2,518 \pm 100$ & $\begin{array}{l}2,712-2,622 \\
(2,345-2,837)\end{array}$ & -2.37 & Wood from $280 \mathrm{~cm}$ below slough surface near base of parting sand. \\
\hline $\begin{array}{l}\text { 3. Drowned YR channel } \\
92 \mathrm{P} 28,384 \mathrm{~cm}\end{array}$ & $\begin{array}{c}\mathbf{2 , 5 6 0 \pm 7 0} \\
\text { Beta-63807 } \\
\text { CAMS-7692 }\end{array}$ & $\begin{array}{c}2,738 \\
(2,361-2,781)\end{array}$ & -3.08 & $\begin{array}{l}\text { Pine needles from } 353 \mathrm{~cm} \text { below water surface. Depth based on } 1991 \text { water } \\
\text { levels. Occurs } 20 \mathrm{~cm} \text { above channel gravel. }\end{array}$ \\
\hline $\begin{array}{l}\text { 4. Drowned YR channel } \\
91 \mathrm{P} 46,415-418 \mathrm{~cm}\end{array}$ & $2,750 \pm 86$ & $\begin{array}{c}2,848 \\
(2,743-3,136)\end{array}$ & -3.39 & $\begin{array}{l}\text { Charcoal (hard chunk) in gravel at depth of } 415-418 \mathrm{~cm}, 3.85 \mathrm{~m} \text { below water } \\
\text { level of slough. }\end{array}$ \\
\hline $\begin{array}{l}\text { 5. Drowned YR channel } \\
91 \mathrm{P} 46,415-423 \mathrm{~cm}\end{array}$ & $\begin{array}{c}\mathbf{2 , 7 1 0 \pm 6 0} \\
\text { Beta-63806 } \\
\text { CAMS-7691 }\end{array}$ & $\begin{array}{c}2,781 \\
(2,745-2,948)\end{array}$ & -3.40 & $\begin{array}{l}\text { Wood from upper part of gravel at depth of } 415-423 \mathrm{~cm} \text { in drowned } \\
\text { paleochannel of Yellowstone River about } 1 \mathrm{~km} \text { downstream from Fishing Bridge. }\end{array}$ \\
\hline $\begin{array}{l}\text { 6. West Thumb area } \\
93 \mathrm{P} 3\end{array}$ & $\begin{array}{c}\mathbf{2 , 8 8 0 \pm 6 0} \\
\text { Beta-63809 } \\
\text { CAMS-7693 }\end{array}$ & $\begin{array}{c}2,980 \\
(2,851-3,210)\end{array}$ & -4.3 & $\begin{array}{l}\text { Wood from about } 17 \mathrm{ft} \text { below lake level of culvert across "north" Little Thumb } \\
\text { Creek, West Thumb. Insects indicate wetland environment. Depth } 23.5-24 \mathrm{ft} \\
\text { below road, est. altitude } 7,717 \mathrm{ft} \text {. Wood probably conifer but not pine. }\end{array}$ \\
\hline $\begin{array}{l}\text { 7. Bridge Bay } \\
94 \mathrm{P} 31 \mathrm{~b}, 497 \mathrm{~cm}\end{array}$ & $\begin{array}{c}\mathbf{3 , 5 6 0 \pm 6 0} \\
\text { Beta-78911 } \\
\text { CAMS-17813 }\end{array}$ & $\begin{array}{c}3,835 \\
(3,690-4,036)\end{array}$ & $\begin{array}{c}-3.43 \\
\text { (approx.) }\end{array}$ & $\begin{array}{l}\text { Wood from } 497 \mathrm{~cm} \text {. Upper part of beach sands } 40 \mathrm{~cm} \text { thick in } 2.6 \mathrm{~m} \text { water on } 1.8 \\
\mathrm{~m} \text { soft lake sediments and above firm lake sediments. }\end{array}$ \\
\hline $\begin{array}{l}\text { Pelican Creek drowned valley } \\
\text { PC5, 26-27.5 ft }\end{array}$ & $11,720 \pm 60$ & $\begin{array}{c}13,805 \\
(13,446-15,123)\end{array}$ & $-4.3 ?$ & $\begin{array}{l}\text { Carbonized plant fragments or roots. From highway boring at Pelican Creek at } \\
\text { depth of } 26-27.5 \mathrm{ft} \text { and about } 16 \mathrm{ft} \text { below present lake level. Sample in lower part } \\
\text { of fill near edge of drowned valley of Pelican Creek. }\end{array}$ \\
\hline \multicolumn{5}{|c|}{ C. Samples lower and younger than S2 and mostly older than submerged shoreline samples } \\
\hline $\begin{array}{l}\text { Lodge Point sand } \\
97 \mathrm{P} 46 \mathrm{~A},+85 \mathrm{~cm}\end{array}$ & $\begin{array}{c}\mathbf{1 3 0} \pm \mathbf{5 0} \\
W W-1640\end{array}$ & $\begin{array}{c}2,261 \\
(0-291)\end{array}$ & 3.7 & Charcoal, probably intrusive from above. \\
\hline
\end{tabular}




\section{Location, sample identifier, depth}

Samples num 1 to 23 are

plotted in fig. 4

Age (yr B.P.)

Corrected age (yr)

Meters above

datum

Remarks

C. Samples lower and younger than S2 and mostly older than submerged shoreline samples—Continued

Lodge Point sand

97P46F, +65 cm

97P46, $45 \mathrm{~cm}$

8. Lodge Point soil that

postdates $\mathbf{S 2}$

95P7, $185 \mathrm{~cm}$

\section{Lodge Point soil that postdates $\mathrm{S} 2$}

95P7, $198 \mathrm{~cm}$

10. $97 \mathrm{P} 46,10 \mathrm{~cm}$

95P9, 190-200 cm

$\begin{array}{cr}\mathbf{2 , 9 8 0 \pm 5 0} & 3,083-3,205 \\ \mathrm{WW}-1638 & (2,968-3,335\end{array}$

$2,870 \pm 40$

WW-1845

$\mathbf{4 , 1 6 0 \pm 6 0}$

WW-564

CAMS-23265

$4,710 \pm 60$

WW-521

CAMS-22090

$5,300 \pm 40$

WW-1846

$4,110 \pm 60$

WW-565

CAMS-23266

CAMS-23266

\section{Pelican Cr. terrace}

truncating $\mathbf{S 2}$

95P15, 105-110 cm

12. Shoreline below $S 2$

(S 1.6)

97P32B, $70 \mathrm{~cm}$

13. Archeological excavation

on S2

S568/E432

14. S2 on Lodge "bay"

95P4, $94 \mathrm{~cm}$

15. S2 on Lodge "bay"

96P50, $53 \mathrm{~cm}$

\section{$6,740 \pm 90$}

Beta-65468

CAMS-8671

$\mathbf{6 , 8 2 0 \pm 5 0}$

WW-1639

\section{$6,800 \pm 90$}

Beta-65467

CAMS-8670

$\mathbf{7 , 2 1 0 \pm 5 0}$

WW-563

CAMS-23264

$\mathbf{7 , 2 1 0 \pm 6 0}$

WW-1174

\section{2,962}

$(2,868-3,158)$

$4,650-4,810$

$(4,451-4,845)$

5,333-5,466

$(5,310-5,591)$

5,998-6,167

$(5,937-6,196)$

4,572-4,778

$(4,423-4,831)$
$(7,432-7,745)$

7,666
$(7,574-7,746)$

7,621-7,660

$(7,493-7,791)$

7,979-8,008

$7,979-8,008$ $(7,875-8,168)$

\section{S3(?) behind FB}

General Store

$\mathbf{7 , 5 6 5} \pm 70$

N248-9/W126, level 10

Beta 63092

ETH-10616

3.4 Charcoal near top of fine-bedded sand. Pulse of well-bedded sand into paleo-

lagoon with Lodge Point soil on S2 shoreline. One possibility is seiche of

Yellowstone Lake into basin.

3.2 Charcoal in middle of fine-bedded sand section. Fine-bedded sand deposited rapidly, perhaps during a seiche of Yellowstone Lake.

2.91 Charcoal $20 \mathrm{~cm}$ below top of buried soil that formed after lake dropped from S2 level. Soil developed and then was buried by Lodge Point sand (see above) and by eolian deposits. Age of S2 (here at $5.16 \mathrm{~m}$ above datum) is significantly greater than these three soil ages.

2.96 Charcoal sample from $33 \mathrm{~cm}$ below top of soil postdating S2 shoreline. Soil developed and then was buried while lake was at or below present level. Age of S2 significantly greater than these soil ages.

2.85 Charcoal near base of fine-bedded sand. Overlies soil dated $4,110 \pm 60 \mathrm{yr}$ B.P. nearby. Age may be near 3,000 yr based on continuous well-bedded sand section that includes above two samples at 45 and $65 \mathrm{~cm}$.

2.8 Charcoal from $20-30 \mathrm{~cm}$ below top of buried soil that postdates $\mathrm{S} 2$ shoreline. Age of S2 significantly greater than these soil ages.

17. Bottom of S-meander

$7,968 \pm 118$

D. Samples associated with S-2 shoreline

$\begin{array}{lll}\text { D. Samples associated with S-2 shoreline } \\ 7,587-7,606 & 2.5 & \text { From } 105-110 \mathrm{~cm} \text { depth in paleochannel on Pelican Creek terrace that truncates }\end{array}$

From 105-110 cm depth in paleochannel on Pelican Creek terrace that truncates

\section{North.}

3.1 Charcoal from base of mixed zone; may be limiting age for local 3-m beach at west end of uplift associated with Storm Point (see fig. 15). Also provides minimum age for $\mathrm{S} 2$ beach.

3.9 At depth of $2.6 \mathrm{~m}$ beneath $\mathrm{S} 2$ surface at $4.3 \mathrm{~m}$ above datum at east part of Fishing

$\mathrm{S} 2=4.3$ Bridge peninsula near Pelican Creek terrace (Cannon and others, 1994).

4.07 Charcoal sample dates time of occupation of S-2 shoreline at $5.16 \mathrm{~m}$ above datum

on Lodge Point. Occurs beneath thick molic soil at depth of $94 \mathrm{~cm}$.

4.48 Charcoal in diatomaceous cap of progradational bar deposits of S2 shoreline and provides age for abandonment of S2 shoreline.

E. Possibly associated with S3 shoreline

$8,378 \quad 4.75$ In old ball field behind General Store. Site in Fishing Bridge S4 paleolagoon.

17. Bo 34

$(8,190-8,451)$

In old ball field behind General Store. Site in Fishing Bridge S4 paleolagoon. may have filled into this area from Yellowstone River. 
Table 1. Carbon-14 and other ages associated with levels of Yellowstone Lake, generally in order of increasing age-Continued.

\begin{tabular}{|c|c|c|c|c|}
\hline $\begin{array}{l}\text { Location, sample identifier, depth } \\
\text { Samples numbered } 1 \text { to } 23 \text { are } \\
\text { plotted in fig. } 4\end{array}$ & $\begin{array}{l}\text { Age (yr B.P.) } \\
\text { Lab number(s) }\end{array}$ & $\begin{array}{c}\text { Corrected age (yr) } \\
\text { (2-sigma range) Method A }\end{array}$ & $\begin{array}{l}\text { Meters above } \\
\text { datum }\end{array}$ & Remarks \\
\hline \multicolumn{5}{|c|}{ F. Abandoned S-meander-Continued. } \\
\hline $\begin{array}{l}\text { 18. Bottom of S-meander } \\
\text { Yell } 92-15\end{array}$ & $\begin{array}{c}\mathbf{8 , 0 3 0 \pm 2 4 0} \\
56712\end{array}$ & $\begin{array}{c}9,000 \\
(8,371-9,527)\end{array}$ & 2 & $\begin{array}{l}\text { Charcoal } 2.12-2.20 \mathrm{~m} \text { below pit datum and } 4.2 \mathrm{~m} \text { from pit end. On top of channel } \\
\text { gravel. See below. }\end{array}$ \\
\hline $\begin{array}{l}\text { 19. Bottom of S-meander } \\
\text { Yell 92-14, 92P30 }\end{array}$ & $\begin{array}{l}\mathbf{8 , 2 5 0 \pm 1 3 0} \\
\text { Beta-56711 }\end{array}$ & $\begin{array}{c}9,152-9,263 \\
(8,812-9,528)\end{array}$ & 2.2 & $\begin{array}{l}\text { Charcoal } 2 \mathrm{~m} \text { below surface and } 10-20 \mathrm{~cm} \text { above river gravel. Dates drowning by } \\
\text { rising waters in ponded reach of Yellowstone River. Minimum age for top of } \\
\text { Yellowstone River channel gravels that extend down to }<1 \mathrm{~m} \text { and probably }<0 \mathrm{~m} \\
\text { above datum. Shorelines S3(?) and S2 are younger than sample. }\end{array}$ \\
\hline \multicolumn{5}{|c|}{ G. Samples related to the S4 shoreline } \\
\hline $\begin{array}{l}\text { 20. Fishing Bridge Peninsula } \\
\text { S375/E512 }\end{array}$ & $\begin{array}{c}\mathbf{8 9 4 0 \pm 6 0} \\
\text { Beta-65466 } \\
\text { CAMS-8669 }\end{array}$ & $\begin{array}{c}10,154 \\
(9,795-10,219)\end{array}$ & $\begin{array}{c}7.8 \\
\mathrm{~S} 4=8.2\end{array}$ & $\begin{array}{l}\text { Charcoal at depth of } 1.6 \mathrm{~m} \text { above beach gravels and in lower part of mixed zone } \\
\text { of eolian sand and beach sand. One-meter-square archeological excavation near } \\
\text { easternmost extent of S4 surface at } 9 \mathrm{~m} \text { above datum. From northern end on old } \\
\text { campground loop. Unit S375/E512 (Cannon and others, 1994, their fig. 44). }\end{array}$ \\
\hline 21. S4 in Fishing Bridge area & $\sim 8,800$ to $\sim 9,400$ & $\begin{array}{c}9,790-9,890 \\
10,580-10670\end{array}$ & $9-7.5$ & $\begin{array}{l}\text { Cody Complex points (late paleo-indian) on S4 between Yellowstone River and } \\
\text { Pelican Creek (Cannon and others, 1995). }\end{array}$ \\
\hline \multicolumn{5}{|c|}{ H. Samples associated with hydrothermal-explosion deposits } \\
\hline $\begin{array}{l}\text { Indian Pond } \\
\end{array}$ & $3,090 \pm 50$ & 3,$272 ; 3,337$ & & Charcoal in soil beneath Indian Pond explosion deposit exposed in culvert \\
\hline $96 \mathrm{P} 45,102 \mathrm{~cm}$ & WW-1173 & $(3,082-3,445))$ & & $\begin{array}{l}\text { excavation for highway halfway from Indian Pond east to lake margin. Maximum } \\
\text { age for Indian Pond deposit. On S5 shoreline of Meyer and Locke (1986). }\end{array}$ \\
\hline $\begin{array}{l}\text { Beneath Indian Pond expl. } \\
\text { deposit } \\
98 \mathrm{P} 25\end{array}$ & $\begin{array}{l}4,220 \pm 40 \\
W W-2161\end{array}$ & $\begin{array}{c}4,828 \\
(4,624-4,852)\end{array}$ & & $\begin{array}{l}\text { Charcoal in soil beneath } 2.5 \mathrm{~m} \text { of Indian Pond explosion deposit, } 0.3 \mathrm{~km} \text { east of } \\
\text { Indian Pond and above } S 5 \text { shoreline. Age of explosion deposit } \sim 3 \mathrm{ka} \text {, so this was } \\
\text { an older charcoal fragment in the buried soil. }\end{array}$ \\
\hline $\begin{array}{l}\text { "Little" Storm Point } \\
\text { beneath Indian Pond expl. } \\
\text { deposit } \\
\text { 95P64B, } 32 \mathrm{~cm}\end{array}$ & $\begin{array}{c}\mathbf{3 , 0 8 0 \pm 5 0} \\
\text { WW-725 } \\
\text { CAMS-28373 }\end{array}$ & $\begin{array}{c}3,270 ; 3,330 \\
(3,082-3,386)\end{array}$ & 9.4 & $\begin{array}{l}\text { Humic wetland deposit buried by greenish Indian Pond hydrothermal-explosion } \\
\text { deposit. Sample } 40 \mathrm{~cm} \text { above shoreline platform at } 8.95 \mathrm{~m} \text { above datum. }\end{array}$ \\
\hline $\begin{array}{l}\text { Beneath Indian Pond expl. } \\
\text { deposit }\end{array}$ & $\begin{array}{c}3,500 \pm 250 \\
W-2734\end{array}$ & $\begin{array}{c}3,727 ; 3,825 \\
(3,170-4,501)\end{array}$ & & $\begin{array}{l}\text { Richmond (1976, section } 74) \text { reported this age for a sample beneath diamicton } \\
\text { now recognized as Indian Pond hydrothermal-explosion deposit. Occurs above } \\
\text { Mary Bay explosion deposit. }\end{array}$ \\
\hline $\begin{array}{l}\text { "Little Storm Point" section } \\
96 \mathrm{P} 47,+140 \mathrm{~cm}\end{array}$ & $\begin{array}{c}430 \pm 50 \\
W W-1169\end{array}$ & $\begin{array}{c}505 \\
(323-539)\end{array}$ & & $\begin{array}{l}\text { Charcoal in weak soil in eolian sand } 40 \mathrm{~cm} \text { below surface. Numbers for distance } \\
\text { above }(+) \text { and below (-) arbitrary datum. }\end{array}$ \\
\hline $96 \mathrm{P} 47,+47 \mathrm{~cm}$ & $\begin{array}{c}420 \pm 50 \\
W W-1166\end{array}$ & $\begin{array}{c}502 \\
(319-536)\end{array}$ & & Charcoal in soil in eolian sand $160 \mathrm{~cm}$ below surface. \\
\hline $96 \mathrm{P} 47,0 \mathrm{~cm}$ & $\begin{array}{c}1,780 \pm 40 \\
W W-1164\end{array}$ & $\begin{array}{c}1,707 \\
(1,570-1,820)\end{array}$ & & $\begin{array}{l}\text { Charcoal. Overlies Indian Pond deposit and provides minimum age for deposit } \\
\text { and for base of eolian sand. }\end{array}$ \\
\hline $96 \mathrm{P} 47,-40 \mathrm{~cm}$ & $\begin{array}{c}2,940 \pm 60 \\
W W-1165\end{array}$ & $\begin{array}{c}3,078-3,154 \\
(2,890-3,323)\end{array}$ & 8.95 & $\begin{array}{l}\text { Charcoal. Immediately underlies Indian Pond explosion deposit and provides best } \\
\text { maximum age for deposit. }\end{array}$ \\
\hline $96 \mathrm{P} 47,-70$ to $75 \mathrm{~cm}$ & $\begin{array}{c}5,160 \pm 60 \\
W W-1167\end{array}$ & $\begin{array}{c}5,916 \\
(5,748-6,167)\end{array}$ & & $\begin{array}{l}\text { Charcoal in soil pendant on platform of S4.5(?) shoreline cut across Mary Bay } \\
\text { explosion deposit. }\end{array}$ \\
\hline $96 \mathrm{P} 47,-75 \mathrm{~cm}$ & $3,970 \pm 50$ & 4,419 & & Blackened material including charcoal. Similar to charcoal sample directly above. \\
\hline
\end{tabular}




\begin{tabular}{|c|c|c|c|c|}
\hline $\begin{array}{l}\text { Location, sample identifier, depth } \\
\text { Samples numbered } 1 \text { to } 23 \text { are } \\
\text { plotted in fig. } 4\end{array}$ & $\begin{array}{l}\text { Age (yr B.P.) } \\
\text { Lab number(s) }\end{array}$ & $\begin{array}{l}\text { Corrected age (yr) } \\
\text { (2-sigma range) Method A }\end{array}$ & $\begin{array}{l}\text { Meters above } \\
\text { datum }\end{array}$ & Remarks \\
\hline \multicolumn{5}{|c|}{ Lake bluffs southeast of Indian Pond and east of "Little Storm Point" } \\
\hline & $4,040 \pm 60$ & $4,451-4,521$ & 8.06 & Sample below two buried soils in tree-throw wedge pulled from platform gravels of \\
\hline \multirow[t]{3}{*}{ 95P53, $103 \mathrm{~cm}$} & WW-722 & $(4,412-4,806)$ & & 7-m S4(?) shoreline. \\
\hline & CAMS-28370 & & & \\
\hline & $4,050 \pm 60$ & $4,453-4,524$ & & Charcoal below buried soil in disturbed zone $20 \mathrm{~cm}$ above platform gravels at $\sim 7 \mathrm{~m}$ \\
\hline \multirow[t]{2}{*}{$95 \mathrm{P} 10 \mathrm{~B}, 85 \mathrm{~cm}$} & WW-522 & $4,411-4,813$ & & above datum in bluffs south of Indian Pond and $85 \mathrm{~cm}$ below buried soil. \\
\hline & CAMS-22091 & & & \\
\hline \multirow{3}{*}{ 95P64B, 48-50 cm } & $5,290 \pm 60$ & $5,922-6,166$ & 9.2 & Charcoal fragments in molic buried soil above stone line of platform of $10.6-\mathrm{m}$ \\
\hline & WW-726 & $(5,922-6,271)$ & & S5(?) shoreline that is eroded on Mary Bay explosion deposit. \\
\hline & CAMS-28374 & & & \\
\hline & $5,890 \pm 40$ & $6,678-6,722$ & 8.36 & Charcoal at base of platform gravels very close to the $S 4(?)$ shoreline. Overlain by \\
\hline 98P11 & WW-2157 & $(6,574-6,844)$ & & buried soil and by Indian Pond explosion deposit. From east of 8-ka samples. \\
\hline \multirow{3}{*}{$\begin{array}{l}\text { Indian Pond Creek West } \\
\text { section } \\
95 \mathrm{P} 6 \mathrm{c}, 80 \mathrm{~cm}\end{array}$} & $2,200 \pm 50$ & $2,156-2,298$ & 13 & Charcoal in eolian sand deposit above level of sample 95P51. May be intrusive from \\
\hline & WW-720 & $(2,060-2,340)$ & & above. \\
\hline & CAMS-28368 & & & \\
\hline \multirow{3}{*}{$\begin{array}{l}\text { Indian Pond Creek West } \\
\text { 95P51, } 105 \mathrm{~cm}\end{array}$} & $8,160 \pm 50$ & $9,032-9,124$ & 10 & Charcoal in sheet-bedded sands about $10 \mathrm{~cm}$ above platform gravel of pre-S4 lake \\
\hline & WW-721 & $(9,007-9,394)$ & & level, $\sim 11 \mathrm{~m}$ above datum when Mary Bay explosion deposit predates S4(?) \\
\hline & CAMS-28369 & & & $\begin{array}{l}\text { shoreline and postdates Glacier Peak ash }(11,400 \mathrm{yr} \text { B.P.) and age of } 11,400 \mathrm{yr} \text { B.P. } \\
\text { Scott Elias (Univ. Colorado) obtained on insect in fibrous lacustrine peat deposit. }\end{array}$ \\
\hline \multirow[t]{2}{*}{ Indian Pond Creek West } & $11,400 \pm 90$ & 13,411 & $\sim 4.8 ?$ & Scott Elias (Univ. Colorado) obtained this age on caterpillar mandibles from \\
\hline & CAMS-17388 & $(13,042-13,800)$ & & $\begin{array}{l}\text { lacustrine stringy peat about } 1 \mathrm{~m} \text { above Glacier Peak ash and several meters below } \\
\text { Mary Bay explosion deposit. }\end{array}$ \\
\hline \multirow{4}{*}{$95 \mathrm{P} 66$} & & Lake bluff south of Indian $\mathrm{F}$ & ond and just ea & ast of cemented column \\
\hline & $\mathbf{8 , 1 1 0} \pm 60$ & 9,025 & 7.64 & Charcoal from grass and brush fire above platform gravels at $7.64 \mathrm{~m}$ above datum \\
\hline & WW-727 & $(8,791-9,262)$ & & $\mathrm{S} 4(?)$ shoreline at $\sim 9 \mathrm{~m}$ above datum. Occurs below two phases of Indian Pond \\
\hline & CAMS-28375 & & & deposit. \\
\hline \multirow[t]{2}{*}{ 98P14 } & $8,340 \pm 40$ & $9,328-9,419$ & 7.0 & Charcoal in platform gravel (base $6.9 \mathrm{~m}$, top $7.15 \mathrm{~m}$ ) of nearby $\mathrm{S} 4(?)$ shoreline that \\
\hline & WW-2159 & $(9,150-9,484)$ & & $\begin{array}{l}\text { truncates Mary Bay hydrothermal-explosion deposit and overlies lake sediments } \\
\text { on Mary Bay explosion deposit. }\end{array}$ \\
\hline \multirow[t]{2}{*}{ 98P13 } & $8,210 \pm 40$ & $9,132-9,243$ & 7.0 & Charcoal from platform gravel of S4(?). Site at same position and very near 98P14. \\
\hline & WW-2158 & $(9,027-9,397)$ & & Overlies lake sediments on Mary Bay hydrothermal-explosion deposit. \\
\hline \multirow[t]{2}{*}{ Richmond (1977, section 74) } & $10,720 \pm 350$ & 12,857 & $\sim 5-6 ?$ & Charcoal $2.6 \mathrm{~m}$ above Glacier Peak ash and beneath Mary Bay explosion deposit. \\
\hline & W-2738 & $(11,344-13,437)$ & & \\
\hline
\end{tabular}


Table 1. Carbon-14 and other ages associated with levels of Yellowstone Lake, generally in order of increasing age-Continued.

\begin{tabular}{|c|c|c|c|c|}
\hline $\begin{array}{l}\text { Location, sample identifier, depth } \\
\text { Samples numbered } 1 \text { to } 23 \text { are } \\
\text { plotted in fig. } 4\end{array}$ & $\begin{array}{l}\text { Age (yr B.P.) } \\
\text { Lab number(s) }\end{array}$ & $\begin{array}{l}\text { Corrected age (yr) } \\
\text { (2-sigma range) Method A }\end{array}$ & $\begin{array}{l}\text { Meters above } \\
\text { datum }\end{array}$ & Remarks \\
\hline \multicolumn{5}{|c|}{ Lake bluff south of Indian Pond and just east of cemented column-Continued } \\
\hline \multirow[t]{4}{*}{ Glacier Peak ash } & $11,450 \pm 50$ & $\begin{array}{c}13,436 \\
(13,160-13,800)\end{array}$ & \multirow[t]{4}{*}{$\sim 2.8$} & \multirow{4}{*}{$\begin{array}{l}\text { Below MB explosion deposit and beneath S4(?) and by inference S5 shoreline. } \\
\text { Collected by Ken Pierce and determined by Andre Sarna to have mixture of } \\
\text { shards of both Glacier Peak and Yellowstone affinities. Probably same ash } 2.92 \mathrm{~m} \\
\text { above datum at Richmond's section (1976, section 74). First two ages from } \\
\text { Whitlock (1993), third from Mehringer and others (1984), and last from Doerner } \\
\text { and Carrara (2001). }\end{array}$} \\
\hline & $12,100 \pm 50$ & $\begin{array}{c}14,100 \\
(13,690-15,360)\end{array}$ & & \\
\hline & $11,200 \pm 50 ?$ & $\begin{array}{c}13,155 \\
(12,910-13,750)\end{array}$ & & \\
\hline & $>11,510 \pm 70$ & $\begin{array}{c}13,460 \\
(13,170-13,820) \\
\end{array}$ & & \\
\hline \multicolumn{5}{|c|}{ Turbid Lake explosion deposit } \\
\hline Turbid Lake explosion deposit & $8,410 \pm 40$ & $9,437-9,469$ & \multirow[t]{2}{*}{$\sim 76 \mathrm{~m}$} & \multirow{2}{*}{$\begin{array}{l}\text { Charcoal from high in bluff of Bear Creek beneath } 2 \mathrm{~m} \text { of Turbid Lake explosion } \\
\text { deposit. Altitude near } 7,800 \mathrm{ft} \text {. }\end{array}$} \\
\hline 98P21B & WW-2160 & $(9,300-9,525)$ & & \\
\hline Turbid Lake explosion deposit & $8,000 \pm 500$ & $8,819-8,986$ & \multirow[b]{2}{*}{ do. } & \multirow{2}{*}{$\begin{array}{l}\text { Sample of charcoal from beneath Turbid Lake explosion deposit along Bear } \\
\text { Creek. Section } 58 \text { of Richmond (1977). Air conditioning problem in lab at time of } \\
\text { analysis. }\end{array}$} \\
\hline 98P21B & W-2486 & $(7,792-10,190)$ & & \\
\hline Turbid Lake explosion deposit & $8,310 \pm 300$ & $9,300-9,398$ & \multirow[t]{2}{*}{ do. } & \multirow{2}{*}{$\begin{array}{l}\text { Sample collected by Dave Love from beneath diamicton now considered to be } \\
\text { Turbid Lake explosion deposit. }\end{array}$} \\
\hline 98P21B & W-1944 & $(8,435-10,150)$ & & \\
\hline \multicolumn{5}{|c|}{ I. Old lake sediment ages } \\
\hline Bridge Bay Marina & $11,890 \pm 60$ & 13,840 & \multirow{3}{*}{-7.97} & \multirow{3}{*}{$\begin{array}{l}\text { Twigs from } 8.97 \mathrm{~m} \text { below platform }(0.27 \mathrm{~m} \text { above water surface on } 8 / 5 / 94) \text { with } \\
\text { beach(?) sand at about } 4 \mathrm{~m} \text { below datum. }\end{array}$} \\
\hline $94 \mathrm{P} 23,897 \mathrm{~cm}$ & Beta 78910 & $(13,624-15,250)$ & & \\
\hline & CAMS-17812 & & & \\
\hline Lodge Point & $13,040 \pm 90$ & 15,678 & \multirow[t]{2}{*}{$\sim 3 ?$} & \multirow{2}{*}{$\begin{array}{l}\text { Sedge peat } 30-40 \mathrm{~cm} \text { below diatomite identified by Ed Theriott (Academy of } \\
\text { Natural Sciences, Philadelphia). Old carbon dioxide effect? }\end{array}$} \\
\hline Yell 92-13 & Beta-56710 & $(14,605-16,173)$ & & \\
\hline \multirow[t]{2}{*}{ Lodge bay } & $13,360 \pm 320$ & 16,053 & \multirow[t]{2}{*}{$\sim 3 ?$} & \multirow{2}{*}{$\begin{array}{l}\text { Sedge peat, Lodge Point, collected by Wayne Hamilton (NPS, retired). Old carbon } \\
\text { dioxide effect? }\end{array}$} \\
\hline & Beta-40764 & $(14,622-16,928)$ & & \\
\hline \multicolumn{5}{|c|}{ J. Samples from southern part of Yellowstone Lake } \\
\hline \multirow{2}{*}{$\begin{array}{l}\text { 22. 00P52 and archeological } \\
\text { site } 48 Y \text { E409 }\end{array}$} & $9,360 \pm 60$ & 10,570 & \multirow[t]{2}{*}{5.8} & $\begin{array}{l}\text { llowstone Lake } \\
\text { Charcoal from base of mixed zone on shoreline gravels that are the } 7.0-\mathrm{m} \mathrm{S} 4 \text { of }\end{array}$ \\
\hline & Beta-148567 & $(10,294-10,737)$ & & $\begin{array}{l}\text { Bill Locke (Montana State Univ., written commun., 2000). Site } 1 \mathrm{~km} \text { east of Grant } \\
\text { Village sewage disposal plant. }\end{array}$ \\
\hline $\begin{array}{l}\text { 23. Osprey Beach site } \\
\text { 48YE409 }\end{array}$ & $\sim 8,800$ to 9,400 & $\sim 9,800$ to 10,300 & $\sim 5.8$ & $\begin{array}{l}\text { Same as 00P52. Point types of late paleo-indian age in the } 8,800-9,400 \mathrm{yr} \text { B.P. } \\
\text { time range. Cody Complex points in base of mixed zone above beach gravels to } \\
5.7 \mathrm{~m} \text { above datum on } 7.4-\mathrm{m} \mathrm{S} 4 \text { of Bill Locke (Montana State Univ., written } \\
\text { commun., 2000). }\end{array}$ \\
\hline $\begin{array}{l}\text { Plainview projectile point from } \\
\text { Osprey Beach site. }\end{array}$ & $\begin{array}{l}\sim 500 \text { years older than Cody } \\
\text { Complex }\end{array}$ & $\begin{array}{l}\sim 10 \text { to } 11.6 \mathrm{ka} \\
\quad(\text { see above })\end{array}$ & $\sim 5.8 ?$ & $\begin{array}{l}\text { A Plainview point was found either (1) recently slumped to the present beach } \\
\text { (Ann Johnson, NPS, oral commun., 2002) or (2) inland } 30 \mathrm{~m} \text { and on a surficial } \\
\text { linear concentration of archeological material (Don Blakeslee, Univ. Wichita, } \\
\text { written commun., 2002). }\end{array}$ \\
\hline Samples associated with $\mathbf{S 2}$ & $6990 \pm 40$ & $7,790-7,815$ & 5.5 & Wood near base of paleochannel in Yellowstone delta aggraded to $\sim 9 \mathrm{~m}$ above \\
\hline $97 \mathrm{P} 54,215 \mathrm{~cm}$ & WW-1848 & $(7,689-7,933)$ & & $\begin{array}{l}\text { datum or } \sim 7,760-7,765 \mathrm{ft} \text { alt. This landform dams off Trail Lake }(7,751 \mathrm{ft} \text {, see } \\
\text { below). Age is similar to } \mathrm{S} 2 \text { shoreline in NW. part of lake. }\end{array}$ \\
\hline
\end{tabular}




\begin{tabular}{|c|c|c|c|c|}
\hline $\begin{array}{c}\text { Location, sample identifier, depth } \\
\text { Samples numbered } 1 \text { to } 23 \text { are } \\
\text { plotted in fig. } 4\end{array}$ & $\begin{array}{l}\text { Age (yr B.P.) } \\
\text { Lab number(s) }\end{array}$ & $\begin{array}{l}\text { Corrected age (yr) } \\
\text { (2-sigma range) Method A }\end{array}$ & $\begin{array}{l}\text { Meters above } \\
\text { datum }\end{array}$ & Remarks \\
\hline \multicolumn{5}{|c|}{ J. Samples from southern part of Yellowstone Lake-Continued } \\
\hline $\begin{array}{l}\text { Yellowstone River Delta at S2 } \\
\text { level }\end{array}$ & $\begin{array}{l}7,215 \pm 70 \\
\end{array}$ & $\begin{array}{l}7,980-8,010 \\
(7,871-8,17)\end{array}$ & $\begin{array}{l}6.1 \text { (lake } \\
\text { level) }\end{array}$ & $\begin{array}{l}\text { Cathy Whitlock (Montana State Univ.) obtained this age for the base of a core from } \\
\text { Trail Lake. Trail Lake is probably dammed by a delta of the Yellowstone River at } \\
\text { the S2 level. Trail Lake altitude is } 7,751 \mathrm{ft} \text {. }\end{array}$ \\
\hline Eagle Bay & $\begin{array}{l}4,540 \pm 40 \\
\text { ETH-3987 }\end{array}$ & $\begin{array}{c}5,294 \\
(5,046-5,317) \\
\end{array}$ & & Colluvium on fault scarp eroded by S4 shoreline (Locke and others, 1992). \\
\hline \multicolumn{5}{|c|}{ Sites in or near modern Yellowstone River delta } \\
\hline $97 \mathrm{P} 51,51 \mathrm{~cm}$ & $\begin{array}{c}1,570 \pm 40 \\
W W-1847\end{array}$ & $\begin{array}{c}1420-1508 \\
(1,350-1,541)\end{array}$ & $\sim 2$ & $\begin{array}{l}\text { Dead duck delta } 5 \mathrm{ft} \text { above present lake. In kettle lake connected to Yellowstone } \\
\text { Lake northwest of Trail Creek cabin. Local delta top is about } 7,738 \mathrm{ft} \text {. }\end{array}$ \\
\hline 97P56, $56 \mathrm{~cm}$ & $\begin{array}{c}850 \pm 40 \\
W W-1850\end{array}$ & $\begin{array}{c}738 \\
(674-908)\end{array}$ & $\sim 1$ & $\begin{array}{l}\text { Stabilized beach with grass and trees at }(7,739 \mathrm{ft}) \text {, east side of delta. Dates beach } \\
\text { deposition near or slightly above present lake level. }\end{array}$ \\
\hline 97P55, $240 \mathrm{~cm}$ below dune top & $\begin{array}{c}340 \pm 40 \\
\text { WW-1849 }\end{array}$ & $\begin{array}{c}328-431 \\
(301-504)\end{array}$ & $\sim 1$ & $\begin{array}{l}\text { Northern point on modern delta. Dates beach deposition near or slightly above } \\
\text { present lake level. }\end{array}$ \\
\hline
\end{tabular}


defined a triangulated irregular network (TIN). The data were interpolated to a 2 -m-square grid by sampling the triangular facets of the TIN at the grid points. We used the lake-level gage at Bridge Bay Marina, $(0.44 \mathrm{~m})$ when the LIDAR was flown, to calibrate to the datum used in previous studies (see, for example, Meyer and Locke, 1986). The altitude of the zero mark on the gage was measured at 2,356.48 $\mathrm{m}$ in 1985 by level surveys.

\section{Lake-Level Rise After Lake Low 4-3 ka}

\section{Yellowstone Lake}

Many of the lower ends of stream valleys entering Yellowstone Lake appear to be drowned. Several streams, both north and south of the West Thumb Geyser Basin, have drowned valleys upstream from their entrances into Yellowstone Lake, commonly with standing water grading upstream into alluvial wetlands. Two such streams are Big Thumb Creek and Little Thumb Creek (fig. 2). The next stream north of Little Thumb Creek (here called "Little Thumb Creek North") has water standing in a drowned valley on the upstream (west) side of the highway. Highway borings in the center of this drowned valley encountered wood at a depth of $4.3 \mathrm{~m}$ below datum with an age $\sim 3.0 \mathrm{ka}(2,880 \pm 60 \mathrm{yr}$ B.P.; table 1, no. 6). Insect faunae studied by Scott Elias (University of Colorado, written commun., 1993) indicate a shallow wetland environment was associated with the wood. Since this time, the level of Yellowstone Lake has risen about 5-6 m, but this small stream has not yet transported enough sediment to fill in the drowned valley.

Immediately offshore from the West Thumb Geyser Basin, aprons of siliceous sinter around active and inactive hydrothermal vents extend well below lake level. Divers from the National Park Service sampled these vents at depths of 4.9 and $5.5 \mathrm{~m}$ below datum. Analysis of the oxygen-isotope composition show the sinter formed subaerially and not under Yellowstone Lake (Pat Shanks, oral commun., 2000). Shoreline data (Locke, 1986; Locke and Meyer, 1994) also suggest substantial local downwarping in the West Thumb area, although the lower shorelines are poorly defined and poorly correlated there.

We cored Yellowstone Lake about $6 \mathrm{~km}$ southwest of the outlet in the relatively quiet water of Bridge Bay (fig. 2), which is sheltered from wave action generated by the prevailing southwesterly winds. A wood sample from a coarse-sand deposit $5 \mathrm{~m}$ below the surface ( $3.45 \mathrm{~m}$ below datum) yielded an age of $\sim 3.8 \mathrm{ka}(3,560 \pm 60 \mathrm{yr}$ B.P.; table 1, no. 7$)$. The sand is well sorted and $0.5 \mathrm{~m}$ thick, similar to the modern beach. It is underlain by firm deep-water lake sediments and overlain by poorly consolidated, fine, lake sediments. We infer that the sand represents a beach deposit drowned by a lake-level rise after $\sim 3.8 \mathrm{ka}$. This site is $3.2 \mathrm{~km}$ west of the north-trending, down-to-the-east Lake Hotel fault, and thus its submergence is not related to downdropping on that fault.

Two other drowned valleys adjacent to Yellowstone Lake and cored by the Federal Highways Administration indicate low lake levels. (1) Pelican Creek is a drowned valley as suggested by the high, steep, stream-cut scarps that now flank the exceptionally wide, low-gradient flood plain. An aggrading stream will have a wide valley, and the necessity of the aggrading stream at times occupying the valley edge where it can undercut the banks is part of this process. Borings for a proposed causeway across Pelican Creek encountered gravelly sands above finer lake sediments. These gravelly sands extend to a depth of 4 to $5 \mathrm{~m}$ below datum. We interpret the gravelly sand to represent a drowned channel of Pelican Creek eroded into lake sediments. Although no carbon samples were obtained from the gravelly sand, an age of $\sim 13.8 \mathrm{ka}(11,720 \pm 60 \mathrm{yr}$ B.P.; table 1$)$ was obtained from the lake sediments just below the gravelly sands. (2) Sedge Creek is nearly at the caldera margin and has a lower valley similar to Pelican Creek. At Sedge Creek, gravelly sediment extends to $18 \mathrm{~m}$ below datum and is underlain by about $6 \mathrm{~m}$ of fine-grained lake sediment that overlies a lower gravelly material, possibly glacial till or outwash. This may suggest the possibility of a drowned valley extending to 18 $\mathrm{m}$ below datum near the caldera margin at Lake Butte and as distant as possible within the caldera from the central part of the caldera where historic inflation and deflation has been greatest.

\section{Outlet Reach of the Yellowstone River}

The outlet reach of the Yellowstone River (fig. 5) is anomalous and extends from the outlet at Fishing Bridge to Le Hardys Rapids (LHR). The river has recently deposited sandbars in the outsides of meanders, effectively straightening its channel and indicating a substantial reduction in stream power (Meyer, 1986, his fig. 17; Locke and Meyer, 1994). The present river has very low gradient $(0.05 \mathrm{~m} / \mathrm{km})$. For low discharge in September, both surveying (Dan Dzurisin, oral commun., 1993) and LIDAR data indicate a total drop of only $0.25 \mathrm{~m}$ in the $5-\mathrm{km}$ distance from the outlet to just upstream from LHR. During higher discharges in June, the water surface would have a somewhat steeper gradient. The only bedrock forming the channel bed in this reach is at LHR, where an erosionally resistant unit in the Lava Creek Tuff acts as a weir that controls water level in the outlet reach (Hamilton, 1987). Uplift or subsidence of this bedrock threshold therefore has the potential to control both the river gradient and the level of Yellowstone Lake. Downstream from LHR, the river has a gradient of $1.8 \mathrm{~m} / \mathrm{km}$, more than 30 times steeper than above LHR.

Although the river is now essentially a continuous, low-velocity "pool" in the outlet reach, steep, high cutbanks on the outsides of the meanders indicate that the older, more sinuous channel contained an energetic river (fig. 5). These scarps were so actively being undercut that they were unvegetated and served as a source for thick eolian sand now preserved at the top of the bank (fig. 5, see also fig. 8). About $1 \mathrm{~km}$ downstream from the outlet, an outer meander of the old channel forms a slough that is isolated from the present active channel by a sand bar.

A core in this slough encountered $3.5 \mathrm{~m}$ of fine sediment over river gravel at a depth of $4 \mathrm{~m}$ (3.4 $\mathrm{m}$ below datum). The 


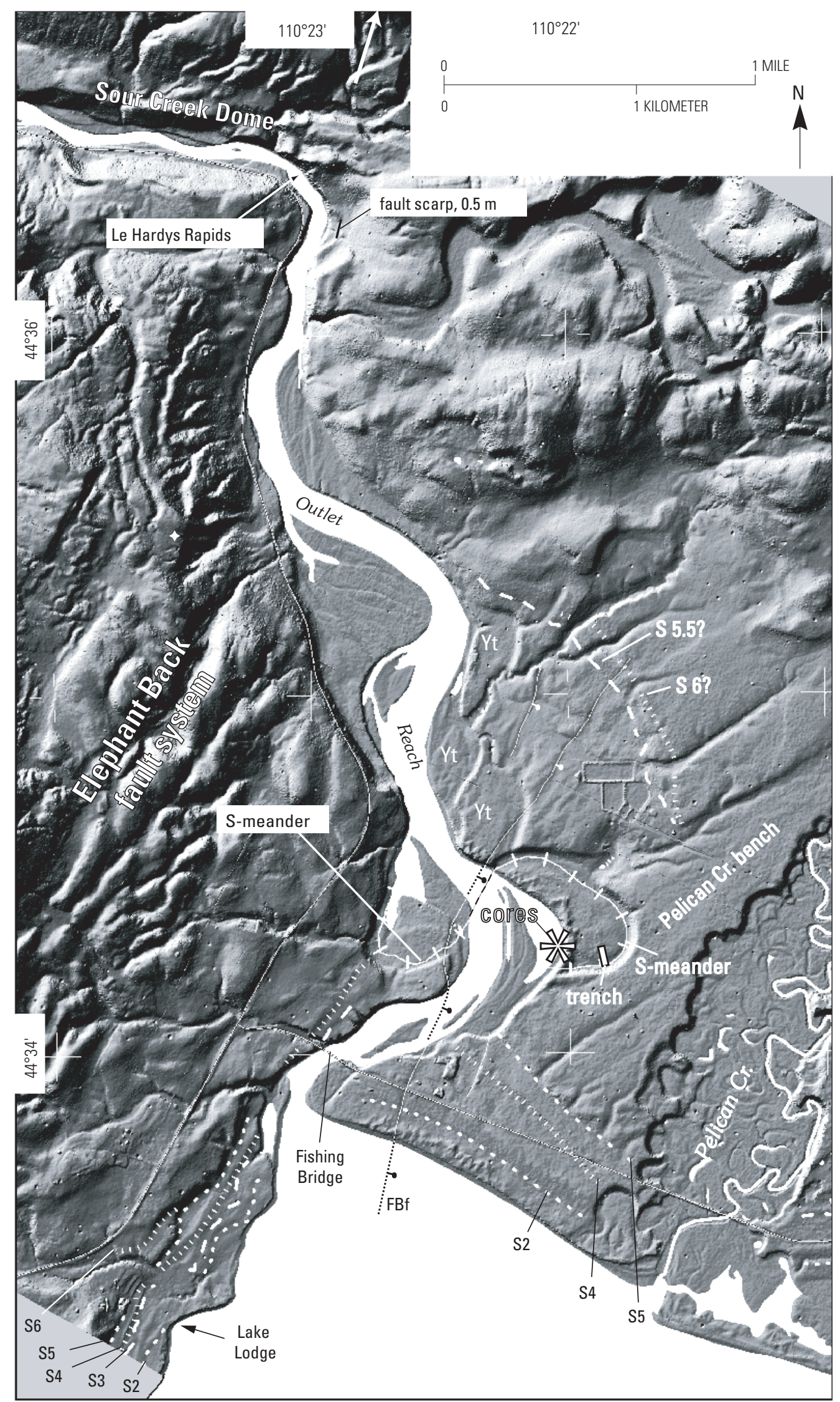

Figure 5. LIDAR image showing the "outlet reach" of the Yellowstone River from the outlet at Fishing Bridge to Le Hardys Rapids. The outlet reach has a drop of only $0.25 \mathrm{~m}$ over a distance of more than $4 \mathrm{~km}$. Sand deposition along the outlet reach indicates the gradient has been diminishing and the channel has been straightening. FBf, Fishing Bridge fault; Yt, Yellowstone River terraces; S2-S6, shorelines. S-meander denoted by thin white line crossed by heavy white bars. LIDAR shown with artificial illumination from the west. 


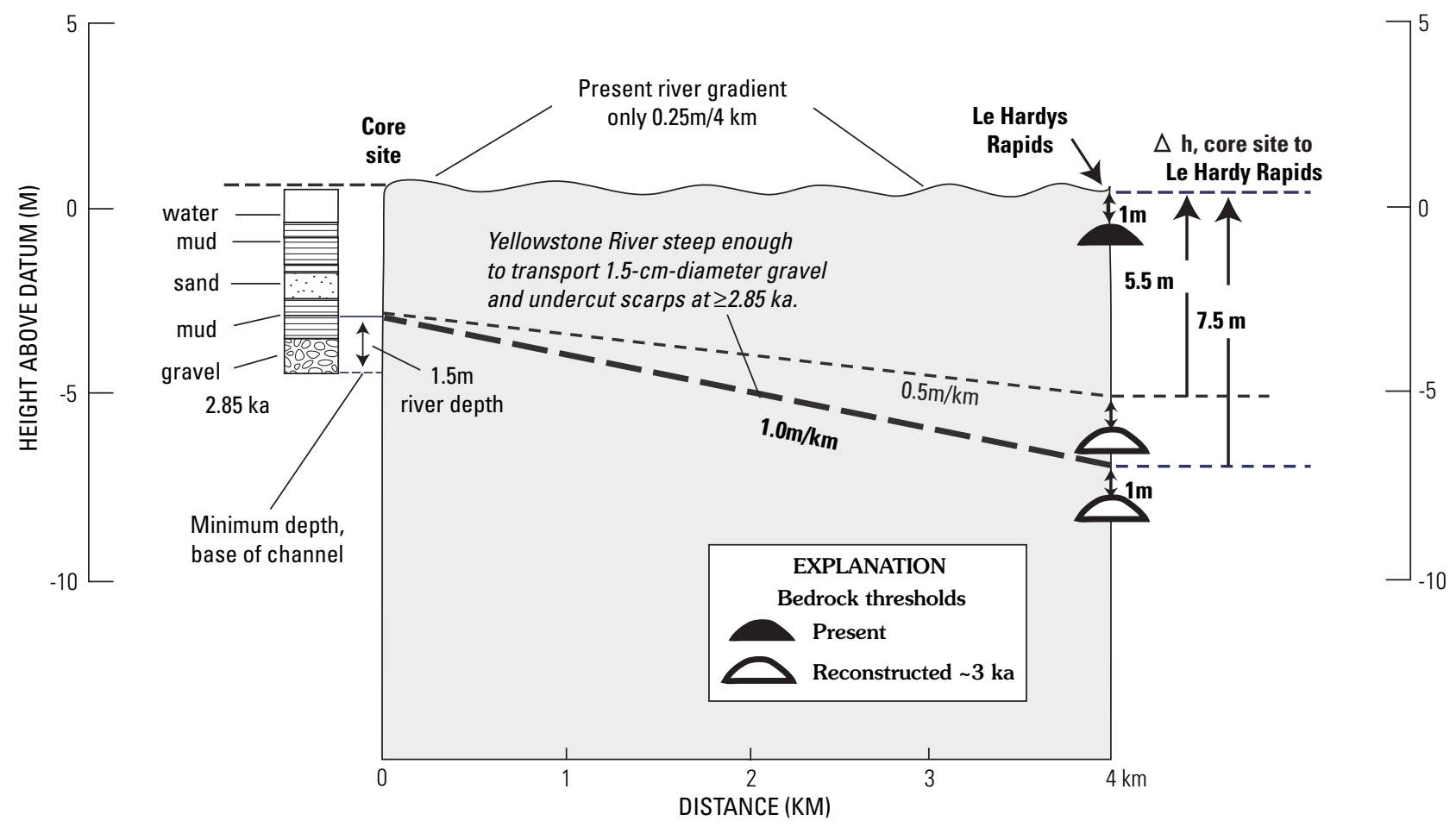

Figure 6. Diagram approximating uplift of Le Hardys Rapids after $3 \mathrm{ka}$. Note that gravel at the core site is below the bedrock threshold at Le Hardys Rapids (LHR). The present drowned profile (upper horizontal wavy line) is compared to a reconstructed profile at $\sim 3 \mathrm{ka}$ when the gradient is estimated to have been $1 \mathrm{~m} / \mathrm{km}$ to the bedrock threshold at LHR (heavy dashed line, see text). At $\sim 3 \mathrm{ka}$, the outlet reach of the Yellowstone River was a vigorous, gravel-transporting, bank-eroding stream.

gravel is at least $1 \mathrm{~m}$ thick; wood and charcoal samples from the upper gravel yielded ages of $\sim 2.8 \mathrm{ka}(2,710 \pm 60$ and $2,750 \pm 86$ yr B.P.; table 1, nos. 4 and 5). We infer that the sedimentary sequence (fig. 6) represents a reduction of gradient and velocity because of relative uplift of the bedrock threshold at LHR downstream, and the fine sediment has accumulated since abandonment and drowning of the channel bend. The top of the river gravel is now about $3 \mathrm{~m}$ below the threshold at LHR, whereas at the time of deposition it would have been significantly above the threshold.

Therefore, at the time of active transport of the river gravels, the Yellowstone River had a considerably steeper gradient and sufficient energy to undercut banks $15 \mathrm{~m}$ high. Based on median sediment diameter of $1.5 \mathrm{~cm}$ and $1.5-\mathrm{m}$ water depth, Waite Osterkamp (written commun., 1996) used the Shields equation to estimate the paleo-river gradient of $1 \mathrm{~m} / \mathrm{km}$ for significant transport of $1.5-\mathrm{cm}$ gravel and $0.5 \mathrm{~m} / \mathrm{km}$ for incipient transport. Figure 6 shows a graphical solution for the uplift of LHR relative to the core site. With a gradient of $1 \mathrm{~m} / \mathrm{km}$, uplift is $7.5 \mathrm{~m}$, and if this gradient is extended from the core site to the outlet, uplift of LHR relative to the outlet is $8.5 \mathrm{~m}$. We acknowledge this paleo-river gradient is poorly constrained and only estimated at one site. Consideration of potential errors indicates that the gradient could have been as low as $0.5 \mathrm{~m} / \mathrm{km}$ - a drop of $2.5 \mathrm{~m}$ over the $5-\mathrm{km}$ outlet reach. This yields a minimum estimated uplift of LHR of $5.5 \mathrm{~m}$ relative to the outlet (fig. 6).

\section{S-Meander and Rise of Lake 9.7-8.6 ka}

The "S-meander" is an abandoned (relict) set of meander bends of the Yellowstone River about 1-2 km north of the outlet (fig. 5). The downstream meander bend was partly filled with a sand bar or spit during a rise of Yellowstone Lake (Meyer and Locke, 1986; Locke and Meyer, 1994). LIDAR data and field measurements show this sand spit is offset $1.2-1.8 \mathrm{~m}$ by the Fishing Bridge fault (fig. 7). Data from the S-meander indicate that the former vigorous river at $\sim 9.7 \mathrm{ka}$ was converted to an arm of Yellowstone Lake by $\sim 8.6 \mathrm{ka}$ (fig. 4).

A trench in the S-meander on the east side of the river revealed buried charcoal resting on channel gravels, showing that drowning of the river was underway by $9 \mathrm{ka}$ (fig. 8; table 1 , nos. 17, 18, and 19). The full suite of landforms and dated river and shoreline deposits on both sides of the modern Yellowstone River imply the following sequence: (1) formation of the S-meander with river transport of 3-cm gravel and vigorous bank undercutting to produce steep scarps $15 \mathrm{~m}$ high, (2) loss of current and cessation of gravel transport and deposition of charcoal by $9 \mathrm{ka}$, (3) continued drowning until Yellowstone Lake rose into this area and cut the S3 shoreline into the meander scarp at $5.5 \mathrm{~m}$ above datum, perhaps at $\sim 8.6$ $\mathrm{ka}$, (4) lowering of the lake about $2 \mathrm{~m}$ and cutting of the S2 shoreline at $\sim 4.1 \mathrm{~m}$ above datum, dated nearby at $\sim 8.1 \mathrm{ka}$, and (5) lowering of lake and river to levels below present-day level by $4-3 \mathrm{ka}$ (fig. 4). 


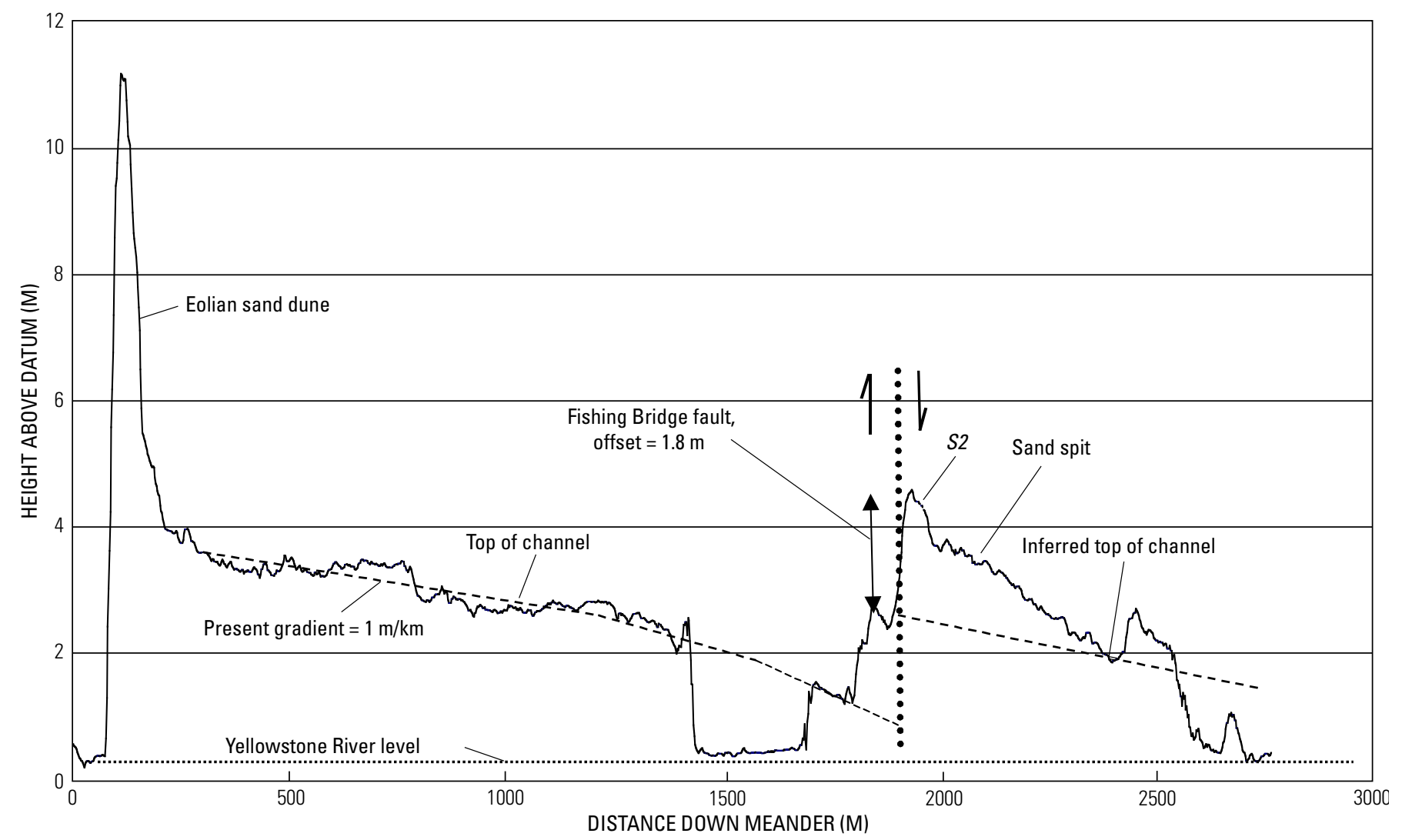

Figure 7. Profile section of the S-meander based on LIDAR data. Position of S-meander shown by line with single crosshatch in figure 5. The meander was drowned, starting by $\sim 9.2 \mathrm{ka}$, and invaded by Yellowstone Lake, including the $\mathrm{S} 2$ sand spit, at $\sim 8 \mathrm{ka}$. The Fishing Bridge fault has offset this sand spit $\sim 1.8 \mathrm{~m}$. The eolian sand dune filled the upper end of the S-meander when the present drowned channel was more active at about $3 \mathrm{ka}$ (see fig. 5). The thalweg of the S-meander has a consistent gradient of about $1 \mathrm{~m} / \mathrm{km}$ across its entire length except where it is offset by the Fishing Bridge fault. The S-meander thalweg now descends below the surface of the outlet reach at the downstream end of the S-meander.

The drowning of the S-meander is a remarkably similar sequence to the more recent drowning of the Yellowstone River reach, particularly the increase in water level to 4-5 $\mathrm{m}$ above channel gravels (compare figs. 6, 7, 8, and 9). We postulate that uplift centered on LHR is also responsible for drowning of the S-meander. A field estimate of the median gravel diameter in the S-meander is $\sim 3 \mathrm{~cm}$, twice that noted in the presently drowned channel that was active about $3 \mathrm{ka}$. A median diameter of $3 \mathrm{~cm}$ with a $1.5-\mathrm{m}$ water depth yields a gradient of $1 \mathrm{~m} / \mathrm{km}$ for incipient movement of pebbles and $2 \mathrm{~m} / \mathrm{km}$ for significant transport of pebbles. We use the conservative $1 \mathrm{~m} / \mathrm{km}$ gradient in figure 9 because some coarser gravel may have been introduced into the stream channel from the high, adjacent cutbank in sandy gravel ("plsg," Pinedale lacustrine sandy gravel of Richmond, 1977). This $1 \mathrm{~m} / \mathrm{km}$ gradient yields uplift of LHR relative to the trench site of $7.3 \mathrm{~m}$ and $8.3 \mathrm{~m}$ relative to the outlet (fig. 9). If we use the $2 \mathrm{~m} / \mathrm{km}$ gradient, then uplift of LHR relative to this $\mathrm{S}$-meander site would be $11.3 \mathrm{~m}$, and $13.3 \mathrm{~m}$ relative to the lake outlet. The S-meander is drowned at its downstream end by the present river, which may reflect a combination of uplift downstream by either warping or faulting, and drowning associated with post-3-ka uplift discussed earlier.

\section{Subaerial Shoreline Sequence, $\sim 8.0$ ka $\mathbf{S 2}$ to 14.4 ka S6}

\section{S2 Shoreline}

Numerous ages on both sides of the outlet date the S2 shoreline (fig. 10) at about $8.0 \mathrm{ka}$ (fig. 4). Directly in front of the Lake Lodge, lagoonal sediments between the wave-cut S2 shoreline and its barrier beach (now partly eroded) are exposed in the present wave-cut bluff. Two charcoal samples from the upper part of these diatomaceous sediments above crossbedded sands yielded ages of $8.0 \mathrm{ka}(7,210 \pm 60$ and 7,210 \pm 50 yr B.P.; table 1, nos. 14 and 15; fig. 4). Other samples from or above a humic soil that is below S2 and clearly postdates it, yield ages of 6.0, 5.5-5.3, 4.8-4.7, and 4.8-4.6 ka ( 5,300, 4,710, 4,160, and 4,110 yr B.P.; table 1, nos. 10, 9, 8, and not numbered) and thus provide additional support for the surprisingly old age of $\sim 8.0$ ka for S2.

On the Fishing Bridge peninsula east of the outlet, a sample from beneath a 2-m-thick eolian sand mantling S2 yielded an age of 7.7-7.6 ka (6,800 990 yr B.P.; Cannon and 
others, 1994, their fig. 40). Just east of this site, a terrace of Pelican Creek truncates S2. A sample from a paleochannel on this terrace yielded an age of $7.6 \mathrm{ka}(6,740 \pm 90 \mathrm{yr}$ B.P.; table 1). We infer an age here for $\mathrm{S} 2$ of $\sim 8 \mathrm{ka}$ or $0.4 \mathrm{k} . \mathrm{y}$. older than this minimum age because following deposition of S2, (1) lake level lowered, and a wide terrace of Pelican Creek formed and eroded out S2, and (2) an active channel on this terrace was abandoned and then accumulated charcoal-bearing sediment about $7.6 \mathrm{ka}$. A mid-Holocene projectile point was collected from the surface of the S2 in the old campground loops D and E (Cannon and others,
1997, their fig. 41h). The eared and basally notched point is similar to those recovered from Layer 30 at Mummy Cave dated $\sim 5$ ka (4,420 \pm 150 yr B.P.; Husted and Edgar, 2002).

Southeast of Yellowstone Lake and $4 \mathrm{~km}$ upstream from the margin of the Yellowstone River delta is a terrace of an older delta with a prominent paleodistributary channel graded to $\sim 7-8 \mathrm{~m}$ above datum (fig. 2). Local damming of this channel has facilitated accumulation of more than $2 \mathrm{~m}$ of organic-rich sediment. Wood from a depth of $2.15 \mathrm{~m}$ just above channel sands in this paleochannel is 7.8 ka $(6,990 \pm 40$ yr B.P.; table 1, part I). Trail Lake (fig. 2) is

S

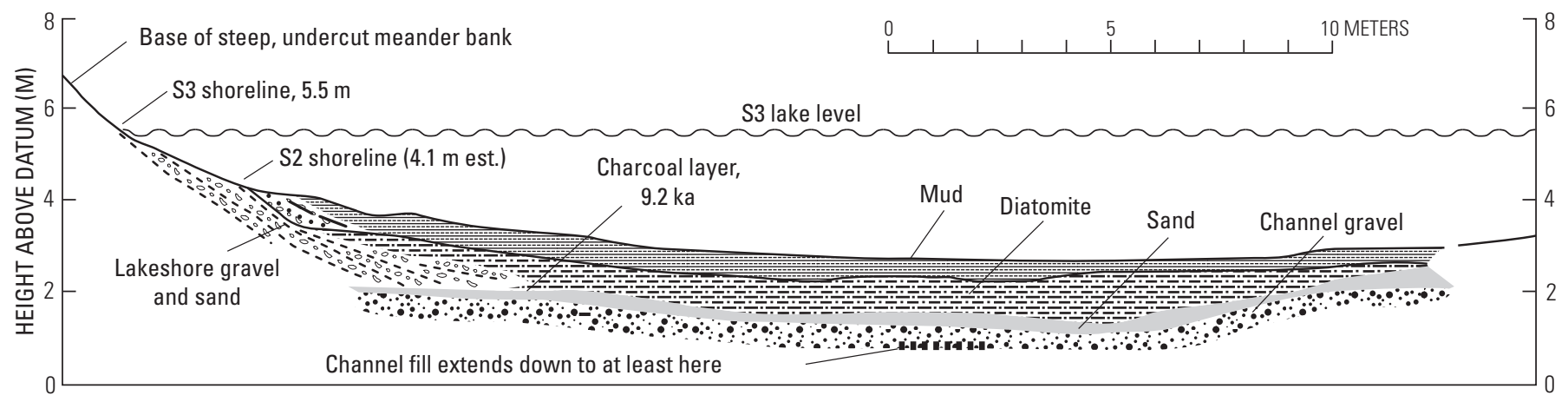

Figure 8. Cross section across the S-meander showing location of channel gravels, $\sim 9.2-k a$ charcoal, and S3 (5.5 m) and S2 (4.1 m) shorelines. This section is based on four nearly connected trenches across the paleochannel labeled "trench" in figure 5.

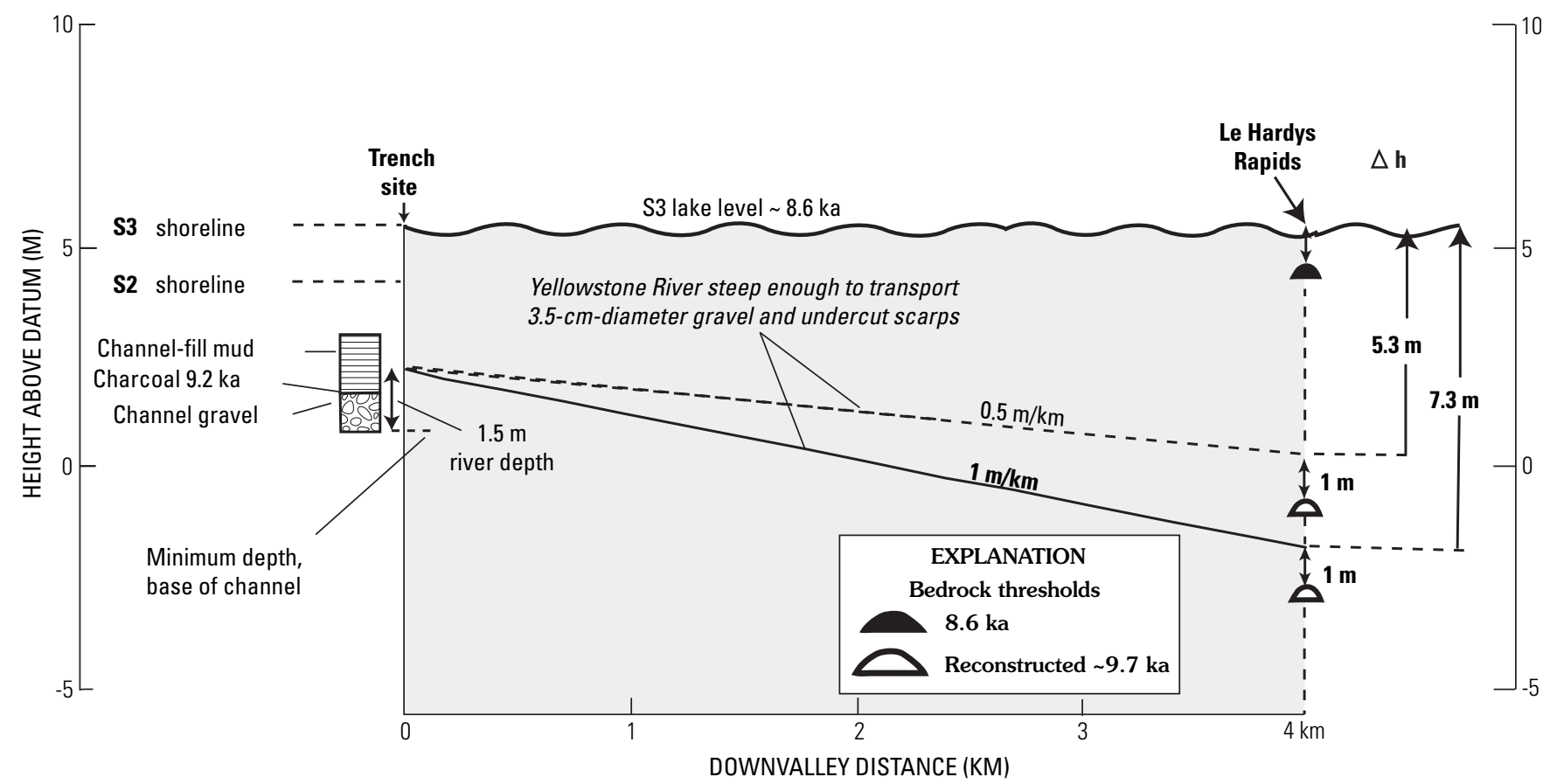

Figure 9. Comparison of outlet reach during S-meander time $(\sim 9.7 \mathrm{ka}$ and gradient of $1 \mathrm{~m} / \mathrm{km})$ with outlet reach during formation of S3 and S2 shorelines (8.6-8.0 ka). This diagram shows uplift of Le Hardys Rapids between $\sim 9.7$ and $~ 8.6$ ka was about $7 \mathrm{~m}$, similar to that between $3 \mathrm{ka}$ and present (fig. 6). The S-meander was converted from a relatively vigorous stream carrying gravel and undercutting its steep banks (fig. 5) to an arm of Yellowstone Lake. 


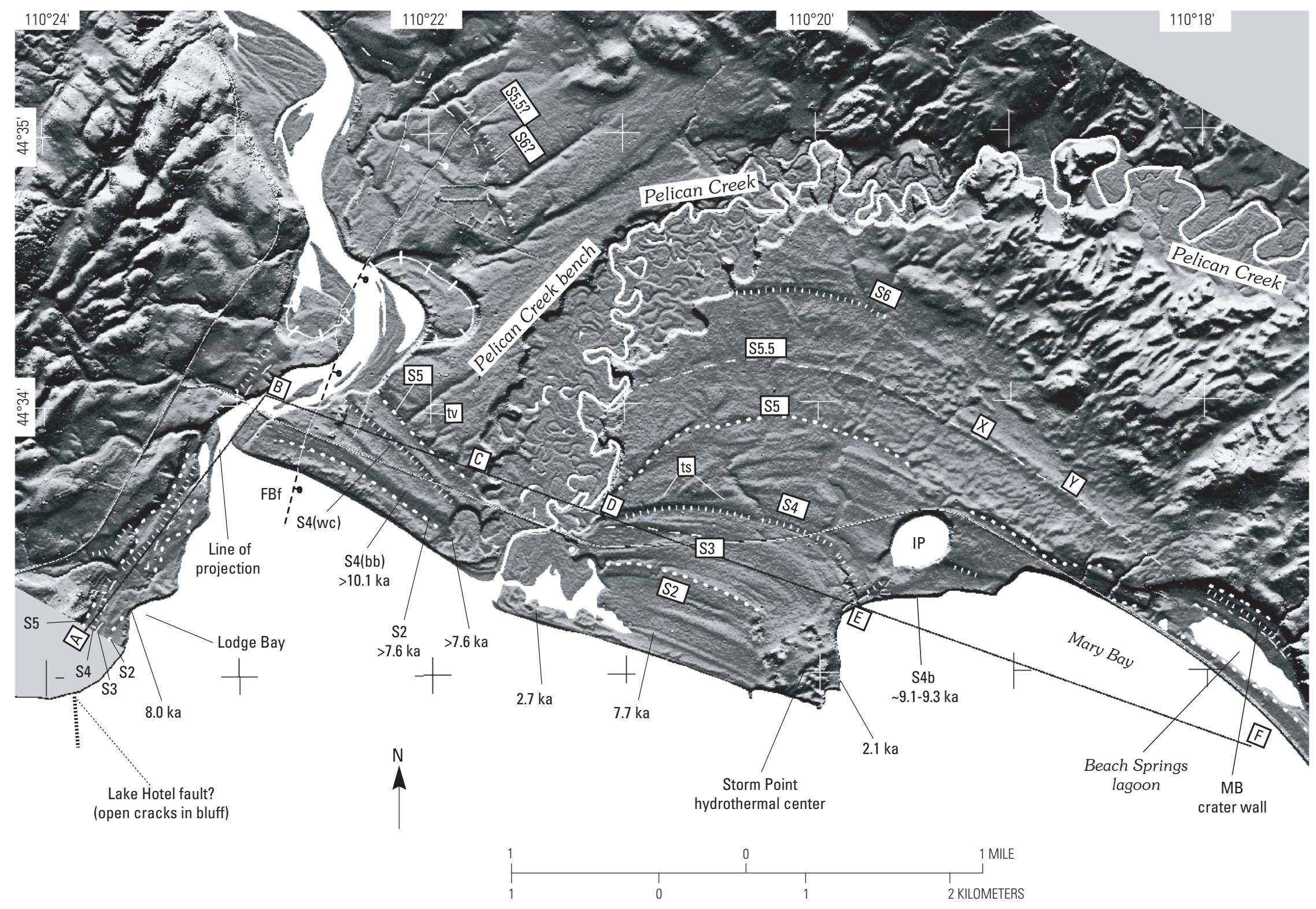

Figure 10. LIDAR image of the northern lakeshore area showing shorelines S2-S6 as well as unlabeled intermediate shorelines. Artificial illumination of LIDAR data from the north. Note surface texture of MB (Mary Bay explosion deposit) northeast of Mary Bay, which is profiled in figure 13 along S5.5 between X and Y. FBf, Fishing Bridge fault; IP, Indian Pond; MB, Mary Bay explosion; tv, truncated valley; ts, truncated shorelines. For S4 both barrier beach (bb) and wave cut (wc) shorelines locally identified, as well as a lower phase near Indian Pond (S4b). ABCDEF, line of projection for figure 12. 
also dammed by this paleodelta. Basal ages from Trail Lake are $8.0 \mathrm{ka}$ (7,215 \pm 70 yr B.P.; Cathy Whitlock, Montana State University, oral commun., 1999). These ages of 8.0 to $7.8 \mathrm{ka}$ are essentially the same as those for the S2 shoreline in the outlet area. Thus, a delta at $\sim 7-8 \mathrm{~m}$ above datum well outside the Yellowstone caldera has similar ages to the $\mathrm{S} 2$ shoreline 4-5 $\mathrm{m}$ above datum in the outlet area. This difference of 2-4 $\mathrm{m}$ over a distance of $34 \mathrm{~km}$ yields an overall tilt of only $0.06-0.1 \mathrm{~m} / \mathrm{km}$ toward the caldera center.

\section{S3 Shoreline}

The S3 shoreline is mapped in the area from the Lake Lodge to the outlet and east of Pelican Creek (fig. 10), but is not well represented on the Fishing Bridge peninsula (Meyer and Locke, 1986). S2 and S3 are best represented in the $S$-meander by the tops of two small deltas at $5.5 \mathrm{~m}$ (S3) and $4.75 \mathrm{~m}$ (S2) above datum that were built into the $\mathrm{S}$-meander by the drainage that comes from the area of the present sewage disposal plant (rectangular dikes shown on fig. 5). S3 is not readily recognizable and seems closely linked with $\mathrm{S} 2$, and is here locally referenced as $\mathrm{S} 2 / \mathrm{S} 3$. Gravelly deposits at the approximate altitude of S3 do occur in the paleolagoon of the S4 shoreline on the Fishing Bridge peninsula. There, at an elevation of $4.75 \mathrm{~m}$ above datum, at a depth of $1 \mathrm{~m}$, and just above well-sorted sands is charcoal with an age of 8.5-8.2 ka (7,565 \pm 70 yr B.P.; Cannon and others, 1994, their fig. 14). Excavations by SUNY-Albany also produced two large side-notched projectile points from the inferred position of the S3 shoreline in the S4 Fishing Bridge paleolagoon (Reeve, 1989, his figs. 13h and 13i). One point is similar to the Blackwater side-notched projectilepoint style recovered from Layer 16 in Mummy Cave and

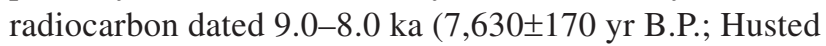
and Edgar, 2002). The depth of the $\mathrm{S} 4$ paleolagoon is such that in S3 time, and perhaps S2 time, Yellowstone Lake may have extended into the northern part of this lagoon.

\section{S4 Shoreline}

On the Fishing Bridge peninsula, a wave-cut shoreline and a barrier beach to the south, with an intervening paleolagoon, represent the S4 shoreline (fig. 10). The General Store at Fishing Bridge lies on the crest of the S4 barrier beach. In the old Fishing Bridge campground area, archeological excavations commonly exposed a meter or more of eolian sand overlying S4 barrier-beach pebbly sand. Charcoal $\sim 10.2 \mathrm{ka}(8,940 \pm 60$ yr B.P.) was collected from 1.6-m depth in disturbed sediment filling the root void of a tree throw in beach gravel. Tree throw occurs when a tree topples to the ground and the tree roots pull up the material held by the roots and thus disturb any stratification present. The beach gravel is covered with $1.3 \mathrm{~m}$ of mixed material-mostly eolian sand (Cannon and others, 1994, their fig. 44).
Two Scottsbluff projectile points and one Cody knife all from the late paleo-indian Cody Cultural Complex have been found on the S4 barrier beach (fig. 11). Ages for the Cody Cultural Complex range from 10.6-9.7 ka (8,800-9,400 yr B.P.; Frison, 1991, his table 2.2) and support an age of $\sim 10.7$ ka for S4. Six additional late paleo-indian projectile points were recovered from excavations on the S4 surface on the Fishing Bridge peninsula. These stylistically variable projectile points conform to lanceolate-projectile-point styles of the Foothills-Mountains tradition that date between 10(?) and 8.8(?) ka (9,000 and 8,000 yr B.P.; Frison, 1992).

On the south shore of West Thumb, similar constraints for the age of S4 are found at the Osprey Beach archeological site (48YE409) $3 \mathrm{~km}$ east of Grant Village (fig. 2). A gravel bench at $6.4 \mathrm{~m}$ above datum extends back to a nearby wave-cut shoreline $\sim 6.8 \mathrm{~m}$ above datum. At a nearby transect, Bill Locke (Montana State University, written commun., 2001, his profile Z54) places S4 at $7.0 \mathrm{~m}$ and S5 at $8.8 \mathrm{~m}$ above datum. Just above beach gravels and beneath $60 \mathrm{~cm}$ of nonbedded mixed material (pebbly sand of mostly eolian origin), charcoal is 10.7-10.3 ka (table 1). Cody Complex material was found in the basal part of the mixed zone just above the beach gravels, as well as in recently slumped material from the wave-cut cliff onto the modern beach below (Shortt, 2001). Cody Complex ages are from 10.6-9.7 ka (see discussion above for age). A Goshen (or Plainview?) point was also found 30 $\mathrm{m}$ back from the bluff (Don Blakeslee, Wichita University, written commun., map, 2002). Goshen (or Plainview?) points are perhaps 500 years older than the Cody Complex, or $\sim 11.6$ to $10 \mathrm{ka}$ (Ann Johnson, National Park Service, oral commun., 2002). This may indicate a slightly older age for this shoreline than S4 in the Fishing Bridge area, although, because occupation of the shoreline occurs after its deposition, the ages also may be the same.

\section{S5 Shoreline}

We trace S5 along the north shore of Yellowstone Lake and along the present outlet reach of the Yellowstone River. LIDAR imagery (figs. 10 and 12) shows that S5 on the east side of Pelican Creek probably extends east to the shoreline that Meyer and Locke (1986) mapped as S6 near Mary Bay. The LIDAR imagery also shows a deposit with hummocky surface texture higher than and north of S5 with a relief of about $1 \mathrm{~m}$ over $50-100 \mathrm{~m}$ (fig. 13). We identify this as the large hydrothermal-explosion deposit from Mary Bay exposed on land and designate it as Mary Bay explosion deposit (MB). In the bluffs south of Indian Pond, MB overlies sandy lake sediment that contains charcoal at $2.6 \mathrm{~m}$ below MB that dates 13.4-11.3 ka (10,720 \pm 350 yr B.P.; Richmond, 1977). The sandy lake sediment grades downward into coarsely varved lake sediment that contains ash $4.6 \mathrm{~m}$ below MB. The ash contains a mixture of shards probably from the ash of Glacier Peak (layers B or G) apparently contaminated by reworked shards from a Yellowstone source (Andre Sarna-Wojcicki, written commun., 1999). Several dated localities place the age of 


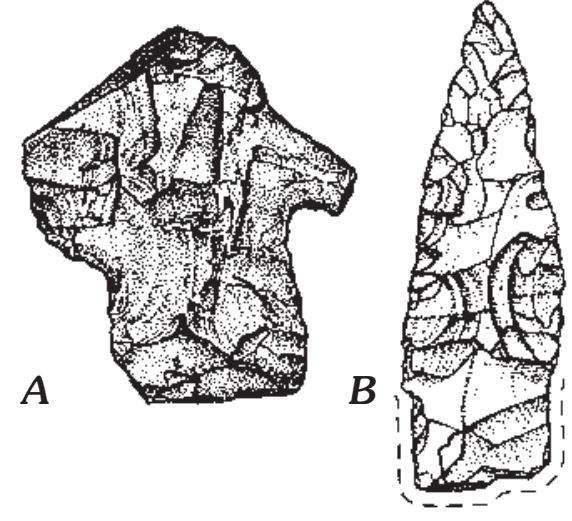

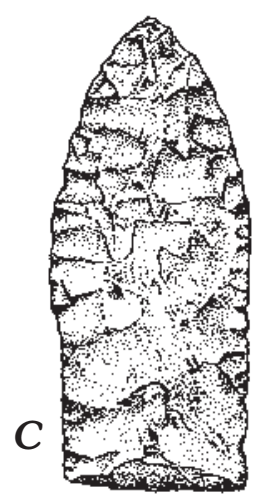

D
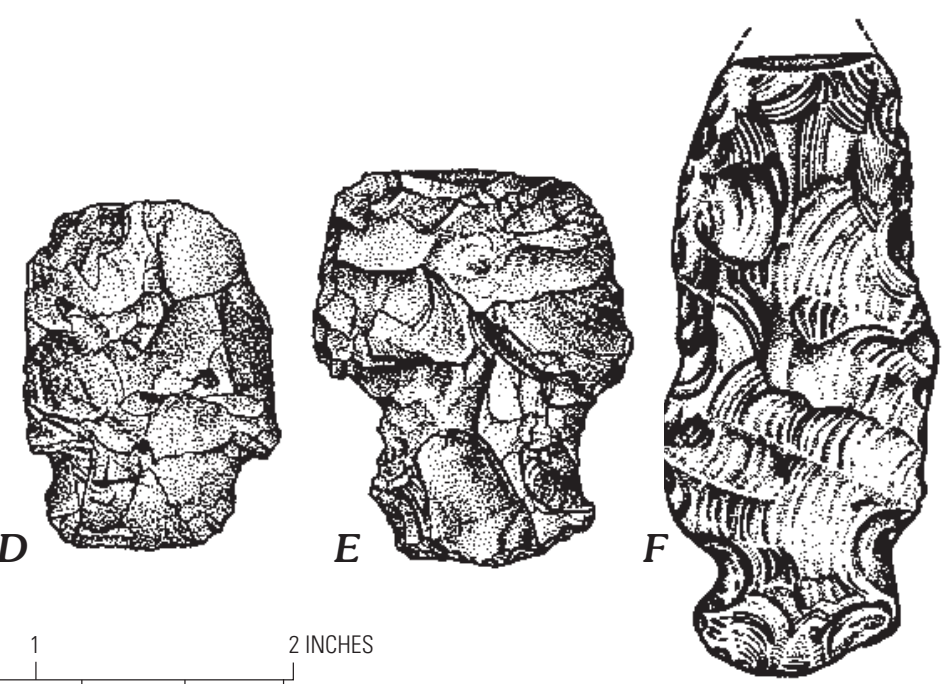

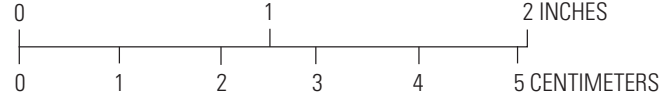

Figure 11. Drawings of projectile points from shorelines on the north shore of Yellowstone Lake. A Cody knife $(A)$ and Scottsbluff projectile points of the Cody Complex (B, C) ( 10.7-9.8 ka) were on S4 deposits on the Fishing Bridge peninsula. Projectile points $D$ and $E$ are late Pleistocene and early Holocene ( 10,000-9,000 yr B.P.; 11.3-10 ka) stemmed points similar in age to Cody Complex points from the S4 paleolagoon. F is a mid-Holocene side-notched point ( 6,000-5,500 yr B.P.; 7-6.4 ka) from the S2(?) barrier beach that encloses the Beach Springs lagoon (fig. 10). It had been weakly abraded by either wind blown sand or wave action.

the ash of Glacier Peak at between 14 and $13.4 \mathrm{ka}$ (table 1). In the Yellowstone-Grand Teton region, Whitlock (1993) obtained ages of 14.1-13.4 ka (12,100-11,450 yr B.P.) for a Glacier Peak ash (table 1). Although a Glacier Peak ash in west-central Montana has been dated as $13.2 \mathrm{ka}(11,200 \mathrm{yr}$ B.P.; table 1) (Mehringer and others, 1984), studies in westcentral Idaho indicate an age older than $13.4 \mathrm{ka}(11,510 \pm 70$ yr B.P.; table 1) (Doerner and Carrara, 2001). Based on the charcoal and ash ages and assuming that the lake sediment accumulated rapidly, we estimate an age of $\sim 13 \mathrm{ka}$ for the MB hydrothermal-explosion deposits and $~ 12.6$ ka for the S5 shoreline (fig. 4). The S5 shoreline is the highest shoreline eroded into the steep crater walls north of Mary Bay (fig. 12, Beach Spring area), which also shows that S5 formed soon after the MB explosion, as S5.5 is not found higher on the crater wall. The S5 shoreline is now tilted down toward the caldera axis (fig. 12). From 16-17 $\mathrm{m}$ above datum on the Mary Bay crater wall, the S5 shoreline descends to about 13 $\mathrm{m}$ above datum on the east side of the Fishing Bridge peninsula and descends to $9 \mathrm{~m}$ across the peninsula.

On the Fishing Bridge peninsula, the S5 shoreline truncates an extensive fill terrace of Pelican Creek (fig. 10, Pelican Creek bench). This terrace postdates S5.5 because this shoreline is not developed on the terrace but is present on both sides (figs. 5 and 10). A narrow valley of a small stream was eroded into this Pelican Creek terrace, but it abruptly terminates at the S5 shoreline (fig. 10). This incised valley and the Pelican Creek bench into which it is incised must have extended farther south to a lower lake level than the S5 shoreline. Lake level then rose (fig. 4), and bluff erosion by the S5 shoreline truncated the stream valley and Pelican Creek bench.

\section{S5.5 Shoreline}

The S5.5 shoreline is a double-crested barrier beach that extends from Mary Bay to Pelican Creek. Hydrothermalexplosion deposits from the MB event mantle the shoreline terrain of Mary Bay to within at least $1 \mathrm{~km}$ of Pelican Creek (fig. 10; see also fig. 13). This mantle extends from just above S5 across S5.5 to above S6. The S5.5 barrier beach shows up clearly through this mantle about $1 \mathrm{~km}$ east of Pelican Creek, where field measurements indicate that the explosion deposits are more than $1 \mathrm{~m}$ thick. Eastward toward the Mary Bay source, the MB explosion deposit thickens until it completely obscures the S5.5 barrier beach about 0.5 $\mathrm{km}$ west of the crater rim (fig. 10).

Shoreline S5.5 becomes totally obscured within $1 \mathrm{~km}$ of the valley of Pelican Creek (fig. 10), apparently by a diamicton locally exposed at the top of Pelican Creek banks. This diamicton may be distal MB or it may be explosion debris that came down Pelican Creek valley. The correlation of S5.5 is less certain west of Pelican Creek because S5.5 is eroded away or buried by the extensive gravel bench along Pelican Creek above the S5 shoreline on the Fishing Bridge peninsula. Northwest of this terrace, S5.5 may correlate to a possible wave-cut scarp east of the Yellowstone River that has a tilt subparallel to that of S5 (figs. 5, 10, and 12). An age for S5.5 of $\sim 13.6 \mathrm{ka}$ is estimated based on its position in the shoreline sequence and the mantle of S5.5 by MB deposits estimated to be $\sim 13$ ka based on relations to Glacier Peak ash and radiocarbon ages on insect parts (fig. 4). 


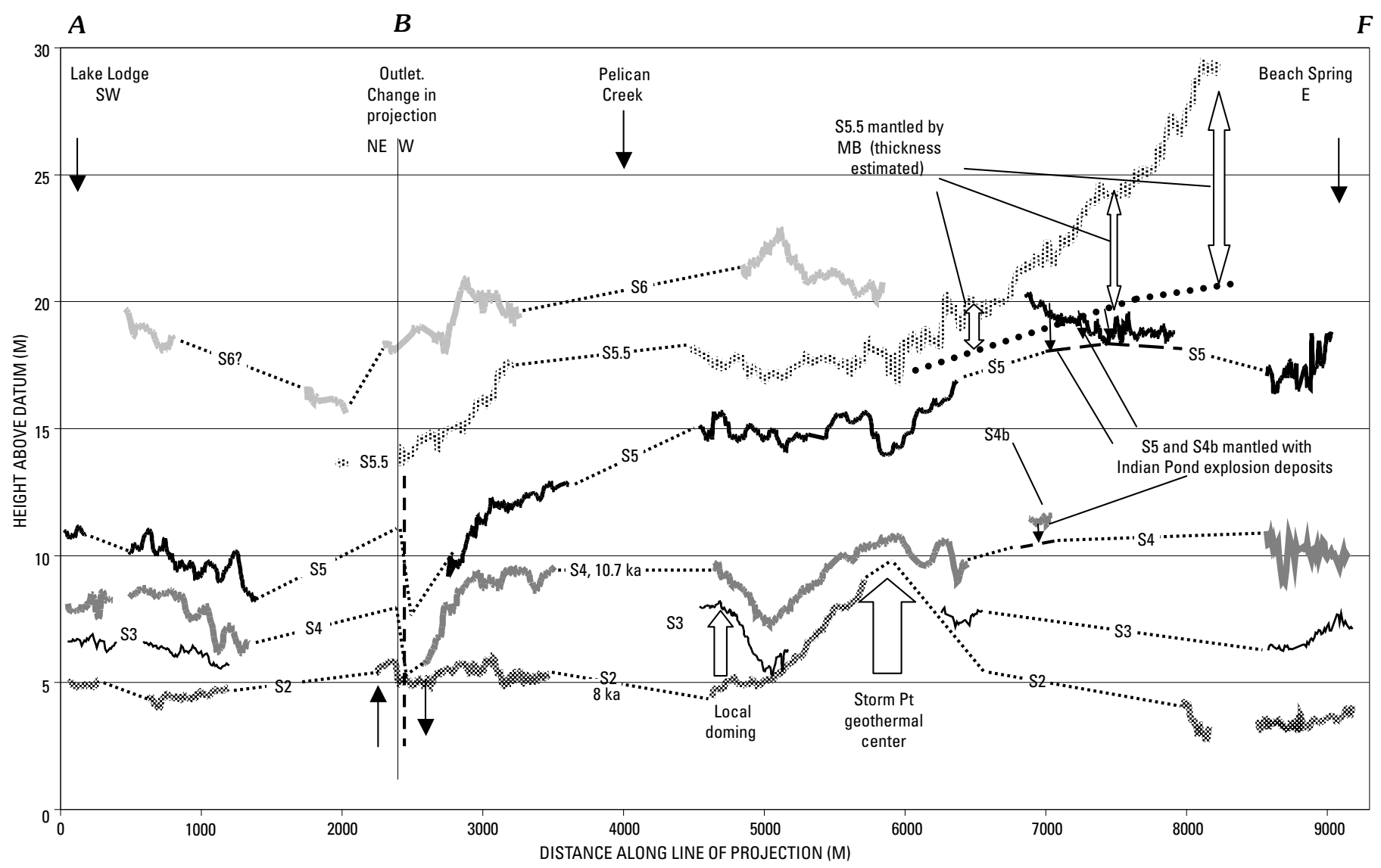

Figure 12. LIDAR profiles of northern Yellowstone Lake shorelines S2-S6. The LIDAR altitude of the mapped shorelines was projected at 10-m spacing onto line ABCDEF of figure 10. S2 is $\sim 8.0 \mathrm{ka}$, S4 is $\sim 10.7 \mathrm{ka}$, and S5 is $\sim 12.6 \mathrm{ka}$. In the outlet area, there is a general sag at the Fishing Bridge fault. Westward from the Mary Bay crater wall at Beach Springs, the S5.5 shoreline is mantled with a decreasing thickness of MB (Mary Bay explosion deposits) (open arrow symbol). The S5 shoreline is mantled with Indian Pond explosion deposits whose thickness was measured at the position of the black arrows westward to where its thickness became too great. Note local doming associated with the Storm Point geothermal center and the smaller dome just east of Pelican Creek (open arrows).

\section{S6 Shoreline}

The S6 shoreline is best expressed just east of Pelican Creek with a well-defined 3-m-high wave-cut scarp (fig. 10). Farther east, a mantle of hydrothermal-explosion deposits obscures it. Assuming an age of about $13 \mathrm{ka}$ for the MB explosion deposit that mantles both S6 and the next lower S5.5, we estimate that the age of S6 is $\sim 14.5-14 \mathrm{ka}$.

\section{Possible Lake-Level Culminations Near 4.5 and $2.5 \mathrm{ka}$}

From the present (S1) time back to the recession from S2 about $8 \mathrm{ka}$, the northwest part of Yellowstone Lake has been near or below its present level. From 4-3 ka, lake and river levels were 5-6 $\mathrm{m}$ below present, but several lines of evidence suggest the lake was near its present level (S1 shoreline) $\sim 4.5$ and $\sim 2.5 \mathrm{ka}$ (fig. 4). Thus, we find rationales for either a simple or more complex lake-level history between $6 \mathrm{ka}$ and $1 \mathrm{ka}$ (fig. 4). The possible culminations near 4.5 and 2.5 ka have some supporting data, but require rather rapid changes in lake level.
Behind the modern barrier beach just east of the mouth of Pelican Creek, charcoal, dated at $2.7 \mathrm{ka}(2,550 \pm 60 \mathrm{yr}$ B.P.; table 1 , loc. 1 , sample 94P33b), was collected at the base of $1.65 \mathrm{~m}$ of eolian sand, $25 \mathrm{~cm}$ above beach gravels. During or following deposition of these beach gravels $\sim 1 \mathrm{~m}$ above datum, Pelican Creek entered the lake to the east of this site. It has subsequently established its inlet west of this site. Rising lake levels to the present shoreline would facilitate this change near $2.5 \mathrm{ka}$. In addition to the Pelican Creek site, the sandy part of the fine-grained section in the core from the drowned Yellowstone River (fig. 6) contains wood $2.37 \mathrm{~m}$ below datum with an age of $2.7 \mathrm{ka}(2,518 \pm 100 \mathrm{yr}$ B.P.; table 1, loc. 2), indicating a river level somewhat below present. In an adjacent core, an age of $2.7 \mathrm{ka}(2,560 \pm 70 \mathrm{yr}$ B.P.; table 1, loc. 3$)$ indicates a maximum age for the sand and a time when the Yellowstone River was near its present level.

In the recently uplifted Storm Point geothermal center, slabby, openwork-platform gravels $3.43 \mathrm{~m}$ above datum contain charcoal dated at $2.1 \mathrm{ka}(2,160 \pm 60 \mathrm{yr}$ B.P.; table 1, section A, sample not numbered). This uplifted beach here is 


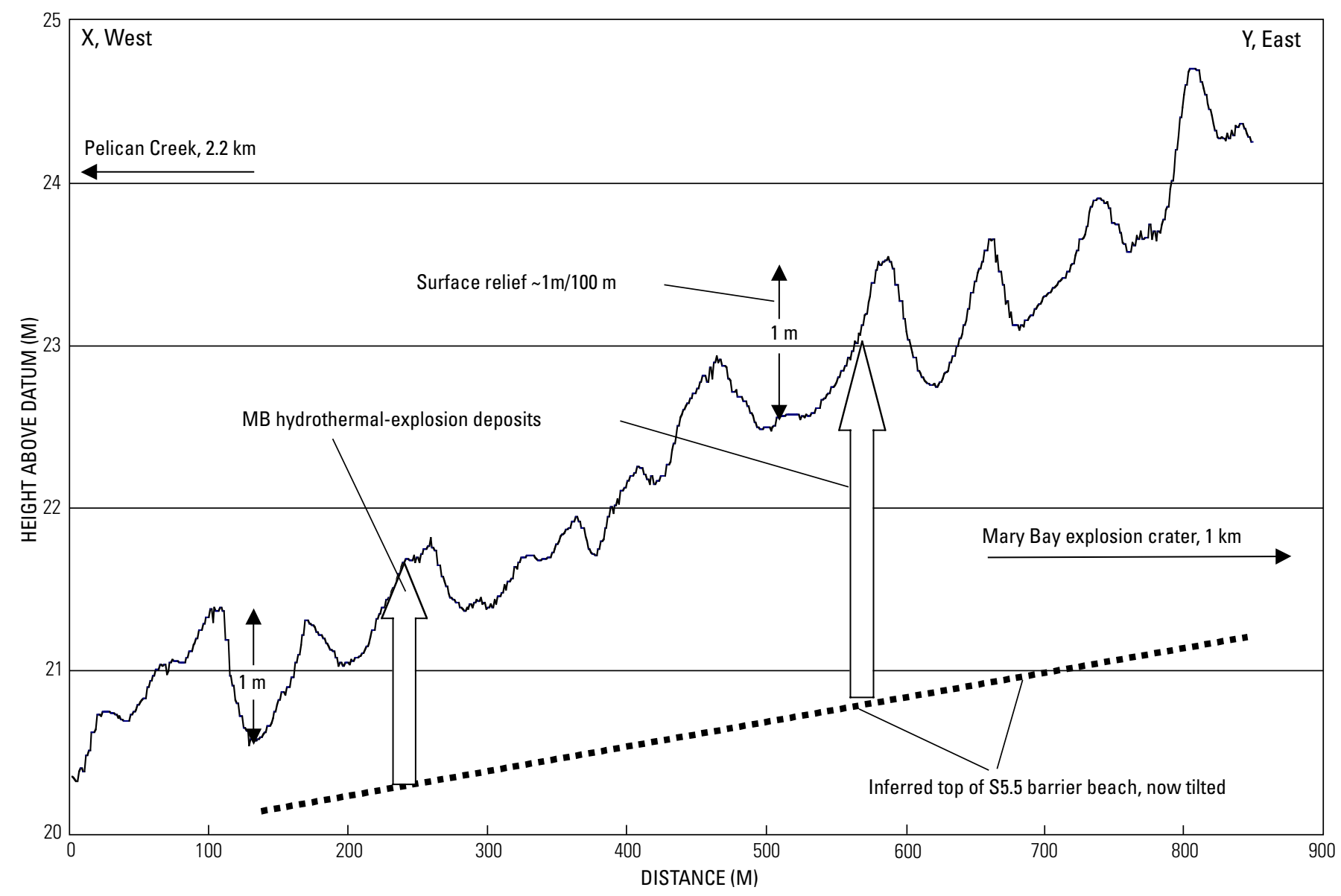

Figure 13. LIDAR profile along S5.5 barrier beach mantled by MB (Mary Bay hydrothermal-explosion deposits). Profile is on barrier beach between $X$ and $Y$ on figure 10. The barrier beach would be smooth (see figs. 14 and 15), but the explosion deposit has a surface texture with an amplitude of $1 \mathrm{~m}$ over a distance of $100 \mathrm{~m}$. The increase in altitude to the east results from an increase in thickness of MB deposits, perhaps supplemented by tilting away from the source in the Mary Bay explosion crater.

distinct from the modern beach, $\mathrm{S} 1$, and also appears to indicate a culmination a little older than $2 \mathrm{ka}$.

About halfway from Lodge Point to the outlet, a relict barrier beach (fig. 5) with a clearly developed soil occurs at the same level as modern storm deposits, including that from high water of 1996 and 1997. The soil has a 12-cm-thick color B-horizon (10YR4/3, moist) and four, 1-cm-thick clay lamellae at depths between 12 and $35 \mathrm{~cm}$, consistent with an age of several millennia. The soil is buried by $35 \mathrm{~cm}$ of modern beach deposits, particularly that of 1996 and 1997. A projectile point recovered from this beach in the collections of the Yellowstone Museum (no. FS-1911) is of the Hanna type. This Northern Plains projectilepoint style was originally defined by Wheeler (1954) and represents a Middle Archaic index artifact dating between 4,500 and $2,800 \mathrm{yr}$ B.P. (Greiser, 1986), suggesting a calibrated age in the $4 \pm 1$ ka range (fig. 4, projectile point symbol at $\sim 4.1 \mathrm{ka}$ ).

In eastern Mary Bay north of Holmes Point, a shoreline $1 \mathrm{~m}$ above modern high water has a tree cover, significant soil development, and artifacts of obsidian flakes, all indicating a lake level just above that of the present. This shoreline is several thousand years old but is younger than $8 \mathrm{ka}$.

\section{Summary of Postglacial Lake-Level Chronology}

Figure 4 plots the history of Yellowstone Lake and Yellowstone River level changes in the northern Yellowstone Lake area over the last $\sim 15,000 \mathrm{yr}$. Both S5.5 and S6 are older than the 13-ka MB explosion deposit and are estimated to be 13.6 and $14.4 \mathrm{ka}$, respectively. Shorelines S6 and S5.5 are younger than deglaciation of the ice cap on the Yellowstone Lake and Yellowstone Plateau area. Above the Yellowstone delta area (fig. 2), Richmond and Pierce (1972) note that a very prominent $~ 18$ - to 20 -m shoreline at Beaverdam Creek correlates by outwash relations with late Pinedale mountain-valley glaciation, somewhat younger than an age of 16.3-14.7 ka (13,140 $\pm 700 \mathrm{yr}$ B.P.; Richmond and Pierce, 1972, their section 14; Porter and others, 1983, their table 4-7, locality E). Locke and Meyer (1994, their fig. 4), however, surveyed the shoreline elevation at this locality to $27.6 \mathrm{~m}$ above datum and correlated it with their S9 shoreline. We note that S7 of Locke and Meyer (1994) in the Southeast Arm may correlate with S5 in the northern Yellowstone area because the LIDAR imagery shows that S5 adjacent to Pelican Creek traces to S7 of Locke and Meyer (1994) near Mary Bay. 
Table 2. Submerged lake and river levels and their normalization to the outlet.

[ $\Delta \mathrm{h}$ is change in height; \#2, \#7, and so forth is the numerical value listed in the row with that number in this table]

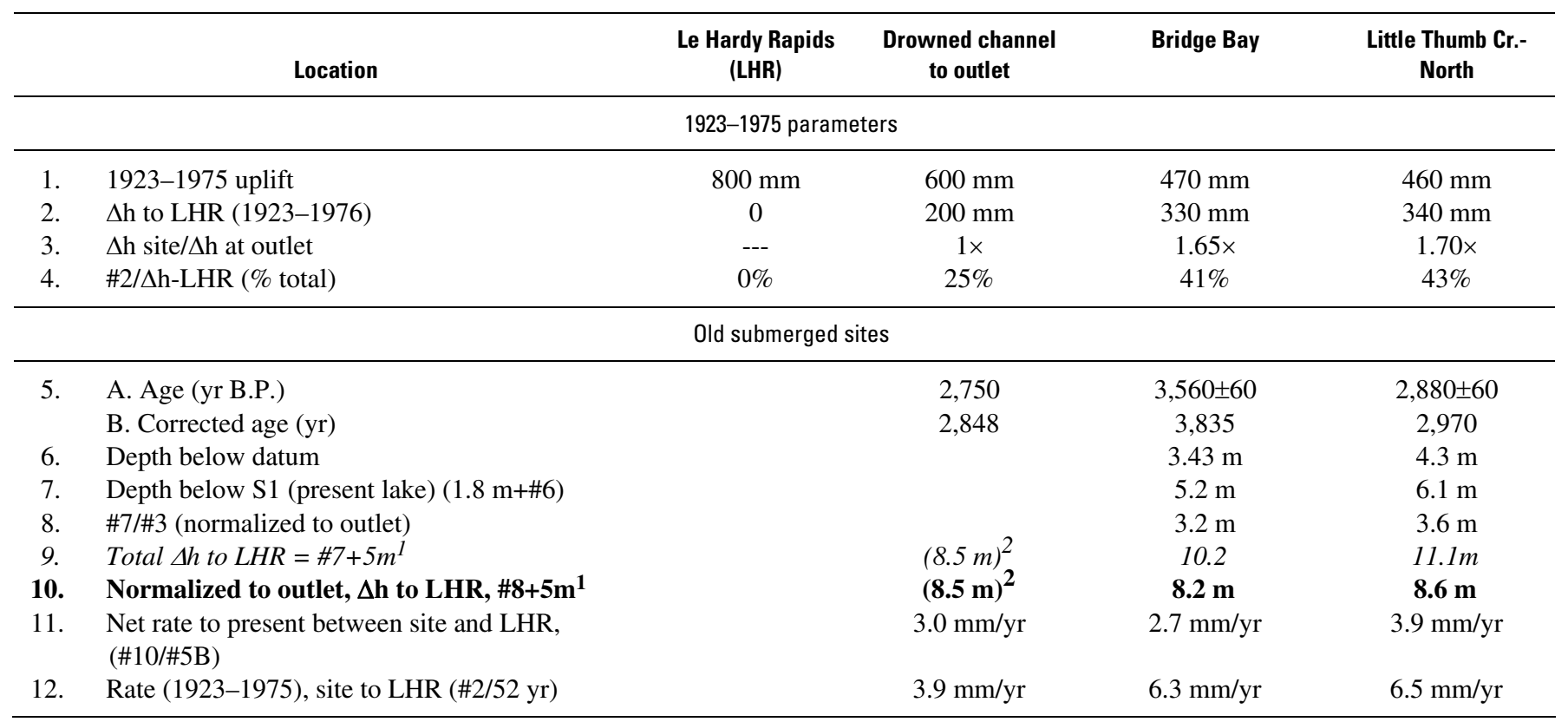

${ }^{1}$ Paleoriver drop of $5 \mathrm{~m}$ from the outlet to Le Hardy Rapids (LHR) based gradient of $1 \mathrm{~m} / \mathrm{km}$.

${ }^{2}$ Estimated uplift as shown in figure 6.

Radiocarbon ages from the Yellowstone Lake area suggest that deglaciation had occurred by $16.2 \mathrm{ka} \mathrm{(>14,000} \mathrm{yr} \mathrm{B.P.;}$ Porter and others, 1983; Whitlock, 1993; Pierce, 2003). At the time of the Deckard Flats readjustment, the ice cap on the Yellowstone Plateau had greatly receded but was probably still present (Pierce, 1979; Sturchio and others, 1994). The radiocarbon chronology of deglaciation is several thousand years older than that produced by cosmogenic surface-exposure dating that places the Deckard Flats readjustment at $\sim 13.8 \pm 0.4$ ka (Licciardi and others, 2001). We cannot resolve why cosmogenic ages are younger than radiocarbon ages, but we suggest either one or both dating systems may be in error.

$\mathrm{S} 5$ is estimated to be $\sim 12.6 \mathrm{ka}$ because it is bracketed as younger than the $\sim 13-\mathrm{ka} \mathrm{MB}$ explosion deposit and older than the $\sim 10.7$-ka S4 (fig. 4). S4 was constructed about 10.7 $\mathrm{ka}$, as dated by Cody Complex projectile points and a ${ }^{14} \mathrm{C}$ age. Subsequent formation of the $\mathrm{S}$-meander indicates a relatively low lake level about $2 \mathrm{~m}$ above the present level at $\sim 9.5 \mathrm{ka}$. S3 is incompletely preserved and is not well dated but was formed after initial drowning of the $\mathrm{S}$-meander and before formation of $\mathrm{S} 2$. S2 is the best dated shoreline at $\sim 8.0 \mathrm{ka}$ based on multiple ages on both sides of the outlet.

After formation of the $\sim 8$-ka S2 shoreline, lake levels have been below or near the present level (fig. 4). Lake level was $\sim 4$ $\mathrm{m}$ below datum from 4-3 ka. Two options are shown for lake levels immediately before and after 4-3 ka (fig. 4): (1) a gradual lowering to 4-3-ka levels followed by a gradual increase to present lake level and (2) possible culminations near the present level $\sim 4.5 \mathrm{ka}$ and $\sim 2.5 \mathrm{ka}$ that bracket low levels between 4 and $3 \mathrm{ka}$ and also define lake-level lows before $\sim 4.6 \mathrm{ka}$ and after 2.2 ka. Both options are shown (fig. 3) with question marks because present information may be interpreted to favor either option. Either option after $\sim 2 \mathrm{ka}$ is consistent with the historic rise of LHR relative to the outlet at a rate of about $4 \mathrm{~mm} / \mathrm{yr}$ (fig. 4).

\section{Uplift of Le Hardys Rapids Indicated by Submerged Localities}

Table 2 shows calculations of the uplift of Le Hardys Rapids relative to sites in the lake as well as at the outlet by normalizing localities to the outlet based on the 1923-1976 uplift dimensions. These calculations indicate between 8 and $9 \mathrm{~m}$ of uplift of the Le Hardys Rapids relative to the outlet following the lowstand 4-3 ka (table 2, line 10). Uplift of LHR relative to the Bridge Bay and Little Thumb Creek North sites is calculated to be 10.2 and $11.1 \mathrm{~m}$, respectively (table 2, line 9). Normalization of these sites to the outlet based on the 1923-1976 uplift pattern yields values of 8.2 and $8.6 \mathrm{~m}$, similar to the $8.5 \mathrm{~m}$ calculated for the outlet (fig. 6). From the lowstand at 4-3 ka to the present, the net rate of uplift is less than the historic rate from 1923-1975 (table 2, compare lines 11 and 12), although this does not consider a possible highstand near $2 \mathrm{ka}$ and possible submergence after that, as shown by the second option in figure 4 . Historic uplift or subsidence of LHR relative to the outlet is only about one-quarter of the total uplift and subsidence relative to the areas beyond the caldera margin (figs. 1 and 3). These rates of $\sim 3-4 \mathrm{~mm} / \mathrm{yr}$, if extended to the caldera margin and multiplied by 4 , would be $12-16 \mathrm{~mm} / \mathrm{yr}$. This is within the range of $16 \mathrm{~mm} / \mathrm{yr}$ for the 1923-1985 uplift as well as for other calderas such as Campi Flegrei, Italy, where rates of 10-20 mm/yr have been observed 
Table 3. Offsets on the Fishing Bridge fault and associated elevation change across the Fishing Bridge peninsula (fig. 5) based on offset shorelines, elevation change due to tilt of shorelines into the fault, and shoreline projections shown in figure 14.

\begin{tabular}{cccccc}
\hline Shoreline/age & $\begin{array}{c}\text { Height above } \\
\text { datum }(\mathbf{m})\end{array}$ & $\begin{array}{c}\text { Elevation } \\
\text { change }^{\mathbf{1}}(\mathbf{m})\end{array}$ & Total offset (m) $\begin{array}{c}\text { Offset/elevation } \\
\text { change }(\%)\end{array}$ & $\begin{array}{c}\text { Interval } \\
\text { offset (m) }\end{array}$ \\
\hline $\begin{array}{c}S 2 / 8.0 \mathrm{ka} \\
S 4 \mathrm{bb} / 10.7 \mathrm{ka}(\mathrm{bb},\end{array}$ & 6 & 0.8 & 0.5 & 63 & 0.5 \\
$\begin{array}{c}\text { barrier beach) } \\
\text { S4wc/10.7 ka }\end{array}$ & 9 & 1.8 & 1.0 & 55 & $0.5(?)$ \\
$\begin{array}{c}\text { (wc, wave-cut) } \\
\text { S5/12.6 ka }\end{array}$ & 9 & $\sim 4.8$ & $\sim 2.9^{2}-3.2^{3}$ & $60^{2}$ & $1.9-2.2$ \\
\hline
\end{tabular}

${ }^{1}$ Elevation change across Fishing Bridge peninsula to Fishing Bridge fault.

${ }^{2}$ Based on fault offset being 60 percent of elevation change as averaged from S2 and S4bb.

${ }^{3}$ Offset based on projection of S4wc and S5 across fault as shown in figure 14.

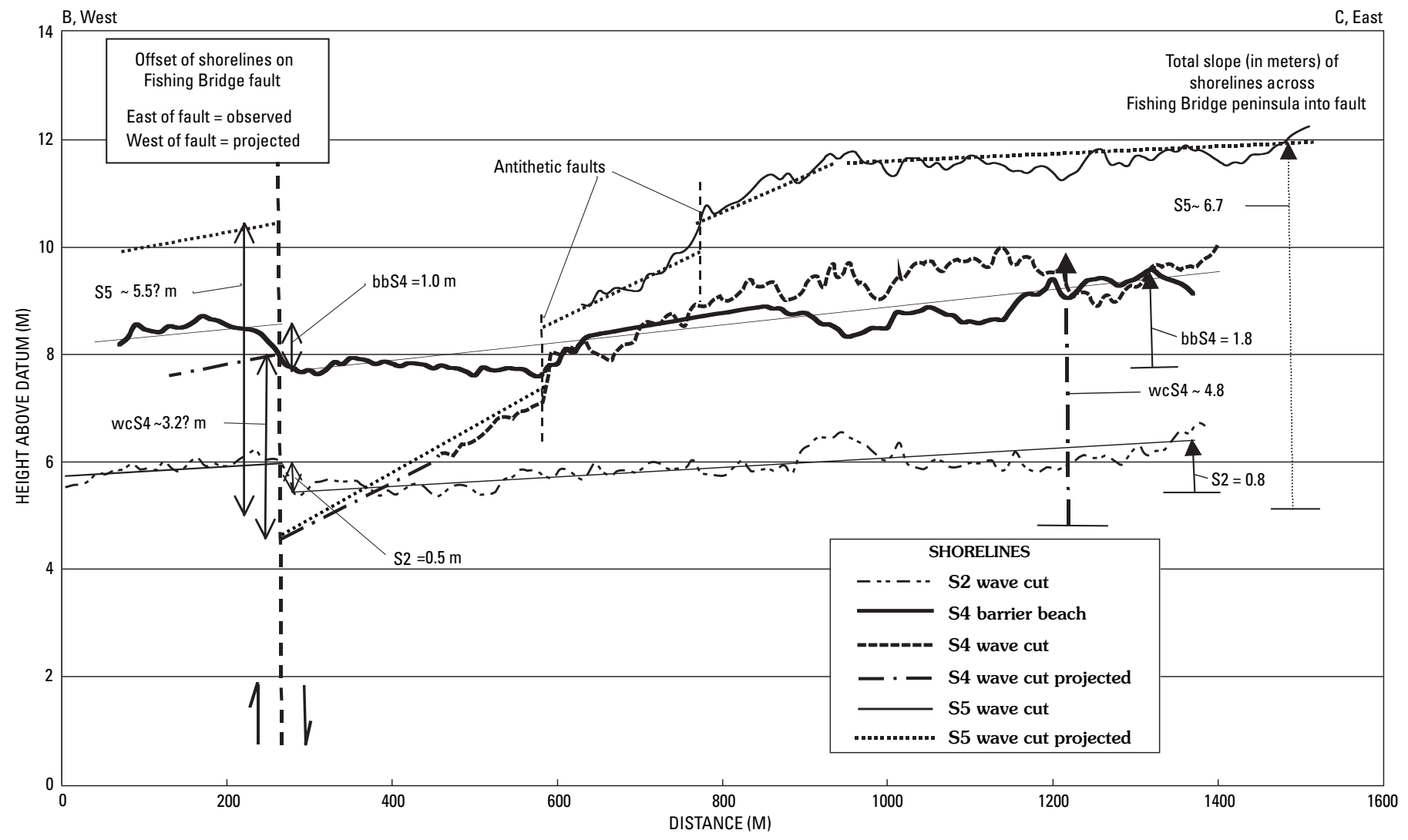

Figure 14. LIDAR profiles showing faulting and tilting of shorelines across the Fishing Bridge peninsula. Location is along line of projection between $B$ and $C$ on figure 10. Four shoreline profiles indicate increased deformation from S2 to S5 time (see table 3). The fault does not cross the S4 or S5 shorelines, but the shorelines show increasing tilt toward the fault with age. S2 is offset $0.5 \mathrm{~m}$. The S4 barrier beach (bbS4) is faulted $1 \mathrm{~m}$, but slightly older wave-cut S4 (wcS4) is projected to be offset $3.2 \mathrm{~m}$ suggesting about $2 \mathrm{~m}$ of offset during S4 time (see text). 
over millennial time scales (Dvorak and Mastrolorenzo, 1991). In a compilation of data for more than 100 calderas worldwide showing signs of unrest, Newhall and Dzurisin (1988) found rates of uplift and subsidence of tens of millimeters to greater than $100 \mathrm{~mm} / \mathrm{yr}$.

\section{Faulting}

Late Quaternary faulting has broken many parts of the Yellowstone Plateau, especially outside of the caldera, but locally within it as well (Christiansen, 2001; Machette and others, 2001). Because of high thermal gradients and shallow young intrusions within the caldera, the brittle-ductile transition may lie at a depth of only 3-5 km, limiting faulting to shallow crustal levels. Compared to sites where the brittle-ductile transition is about $15 \mathrm{~km}$ deep, maximum earthquakes are of lesser magnitude (Smith and Braille, 1993). In contrast to the large subsided half-graben basins and uplifted blocks of adjacent Basin-and-Range terrain (for example, Jackson Hole and the Teton Range), normal-fault systems in the caldera have little net offset and commonly form grabens and fissure-like structures.

The Elephant Back fissure system, the most prominent set of young faults within the caldera, strikes northeasterly from the Sour Creek resurgent dome to east of the Mallard Lake dome (fig. 1). This fissure zone is nearly parallel to the caldera long axis and also forms the axis of historical uplift and subsidence. The fissures of the Elephant Back system may result from general extension or from fracturing associated with localized uplift and subsidence along the caldera axis. These young faults cut the 150-ka Elephant Back flow, but leveling resurveys of benchmarks across the northeast end of the Elephant Back zone show no apparent steps or offsets suggesting structural changes since the first survey in 1923 (Dzurisin and others, 1994; Dan Dzurisin, oral commun., 1995).

LIDAR imagery reveals a fault that offsets postglacial surfaces across the west tip of the Fishing Bridge peninsula and extends $3 \mathrm{~km}$ to the NNE (fig. 5). Figure 14 shows local shoreline profiles, shoreline tilts, and fault offset, which are tabulated in table 3. Only shorelines S2 and the barrier beach of S4 are preserved actually crossing the fault. S2 and the S4 barrier beach at the east end of the Fishing Bridge peninsula are higher than their upfaulted remnant at the west end of the peninsula (fig. 14), although farther eastward S2 and S4 are horizontal (figs. 12 and 14). The ratio of fault offset to total shoreline-elevation change across the Fishing Bridge peninsula produced by tilting into the fault is about 60 percent (table 3, S2 and S4bb). Two methods were used to estimate offset of wave-cut S4 and S5 that do not cross the fault. One is based on the fault offset equaling 60 percent of the tilt into the fault (table 3 ). The other method was a visual projection of the shoreline across the fault (fig. 14) consistent with the shoreline west of the fault (fig. 12). Table 3 outlines the sequence of tilting and surface-offset faulting events.

We infer that a faulting event occurred between erosion of the S4 wave-cut shoreline and construction of the S4 barrier beach on the Fishing Bridge peninsula. Faulting and subsidence of the hanging wall apparently submerged the west end of the wave-cut S4 shoreline. Longshore drift of sediment from Pelican Creek then prograded a barrier beach across the south side of the drowned area to form a lagoon. Because the strongly tilted S4 wave-cut shoreline and much less tilted S4 barrier beach are at the same elevation on the east side the Fishing Bridge peninsula (east side of fig. 14), lake level did not change significantly before construction of the barrier beach, and faulting did not affect the lake outlet level. The S4 interval is the only time during the period of shoreline-terrace formation that a barrier beach and lagoon existed on the Fishing Bridge peninsula. This relationship also links the time of tilting and offset with barrier beach and lagoon formation.

Features in the area of the S-meander are also offset by the Fishing Bridge fault (figs. 5 and 7). A S2 sand spit built across the $\mathrm{S}$-meander is offset $1.8 \mathrm{~m}$ (fig. 7). The higher bench just south of and older than the $\mathrm{S}$-meander is offset $1.2 \mathrm{~m}$ (fig. 5), indicating no increase in offset going back to at least the $~ 9.7-k a$ age of the $\mathrm{S}$-meander. Figure 7 shows that the gradient of the channel of the $\mathrm{S}$-meander is $\sim 1 \mathrm{~m} / \mathrm{km}$ along most of the meander. No other fault strands offset the wide belt occupied by the S-meander. Farther north, a Yellowstone River terrace that predates the S-meander is offset $1.5-1.8 \mathrm{~m}$ by the Fishing Bridge fault (fig. 5). The fault continues northward with surface offsets of $1 \mathrm{~m}$ or less and dies out above the S6 shoreline. Surfaces older than the $~ 9.7-k a$ S-meander on both sides of the Yellowstone River are offset less than $2 \mathrm{~m}$.

An $~ 1-k m$-wide graben is located on the floor of Yellowstone Lake $0.5 \mathrm{~km}$ southeast of the Lake Hotel (fig. 2). Seismic reflection profiles by Otis and others (1977) first revealed this graben, and Kaplinski (1991) mapped it in greater detail. Because of its potential involvement in shoreline deformation, Locke and Meyer (1994) termed this structure the Outlet graben. However, recent detailed mapping with GPS control (Morgan and others, 2003 and this volume; Johnson and others, 2003) shows that it is about $1 \mathrm{~km}$ west of where originally mapped and has a more northwesterly strike. The primary fault on the west side of the graben strikes about N. $10^{\circ} \mathrm{W}$. from the north end of Stevenson Island (figs. 2 and 10). Because it does not appear to intercept the outlet, we call this fault the Lake Hotel fault. No faulting of shorelines has been observed in the Lodge Bay area, but, along the lakeshore in Lodge Bay directly below the General Store parking lot (fig. 10), vertical fractures with 10- to 20-cm openings in compact "lacustrine till" suggest extensional deformation, possibly due to lateral spread. Directly above these fractures, S5 platform gravels at 10-11 m above datum are not offset. Farther north along this trend, the Lake Hotel fault may extend onshore to a prominent escarpment behind the Lake Lodge and continues northward to the outlet area. No evidence of offset of surface sediments has been observed in the field or with LIDAR data, however, and the S5 barrier beach adjoins this escarpment but is not offset (fig. 5). Therefore, any fault offset on this escarpment is older than S5, and in S5 time this was a wave-cut cliff. 


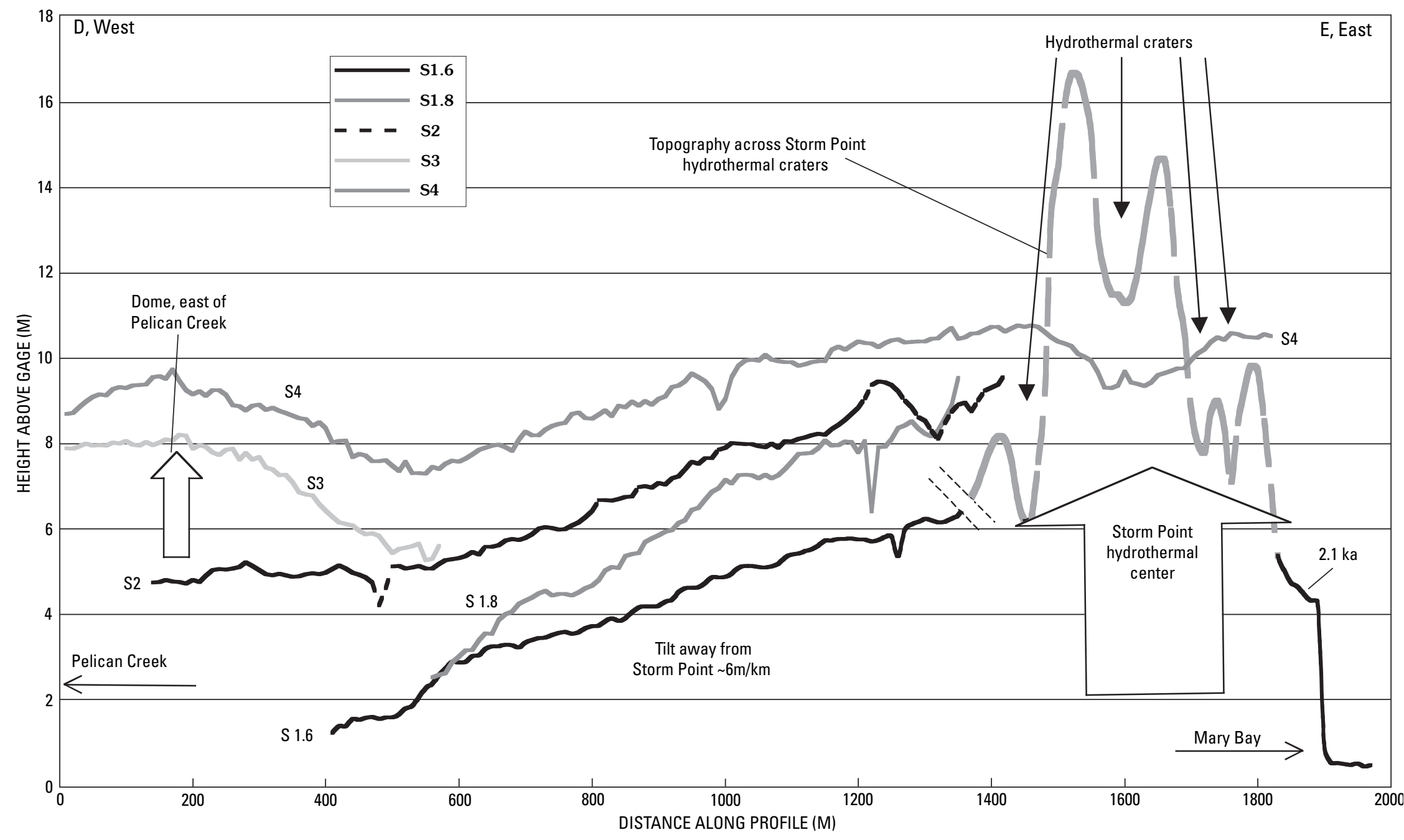

Figure 15. LIDAR profiles of S4, S2, and two younger shorelines (S1.8 and S1.6) from the Storm Point geothermal center west nearly to Pelican Creek. Location is along line of projection between $D$ and $E$ on figure 10 . The shorelines rise as steeply as $6 \mathrm{~m} / \mathrm{km}$ to the Storm Point geothermal center. A local anticline interrupts this westward tilt and is well shown by S4 and S3 just west of Pelican Creek. East of 1,400 m, the dashed broad gray line is a topographic profile that shows the craters of the Storm Point hydrothermal center. 


\section{Local Deformation of Shorelines}

LIDAR data define strong local tilting away from the Storm Point geothermal center (fig. 15). The shorelines that intersect the center (S2, S1.8, and S1.6) show steep subparallel tilts of as much as $6 \mathrm{~m} / \mathrm{km}$. The shorelines that skirt north of the center show lesser tilting; $\mathrm{S} 4$ shows a gentle 4-m-high dome, and S5 is apparently not affected. This tilting resembles Holocene bulges on the floor of Yellowstone Lake inferred to be related to hydrothermal pressures (Morgan and others, 2003 and this volume; Johnson and others, 2003).

A local dome just east of Pelican Creek has about $3 \mathrm{~m}$ of relief in S3 and S4 (fig. 15). Shorelines on the east limb of the dome form a sharp syncline with the shorelines tilted away from the Storm Point hydrothermal center (figs. 10, 12 , and 15). We have observed no hydrothermal features or faulting in the immediate vicinity, but this dome may imply subsurface pressure buildup and merits additional investigation to understand the cause of deformation and any associated hazard. South of the dome, the beach sands on the shore of
Yellowstone Lake are hot at a shallow depth and are the site of a rare thermophilic(?) sand verbena, Abronia ammophila (Whipple, 2001). In February of 2004, water vapor indicated heat just offshore of this beach. This may reflect hydrothermal processes associated with the anticline.

\section{Discussion}

The pattern of both rises and falls of lake level (fig. 4) combined with the historically observed inflation and deflation suggests intriguing volcanic, hydrothermal, and (or) other processes may be involved. Table 4 outlines the many processes for changing Yellowstone Lake and Yellowstone River levels. In the following, we discuss most of these processes emphasizing those most relevant to our study. We attribute the overall decrease in lake level observed during the first half of the record to lowering of the outlet by a combination of erosion and subsidence. We suggest that a millennial-scale oscillation occurred combined with an overall lowering of lake and river levels during the first

Table 4. Processes for decreases, increases, and oscillations in Yellowstone Lake and Yellowstone River levels in postglacial time.

[The pattern of historic changes and the present drowned "outlet reach" of the Yellowstone River suggest that changes in the altitude of Le Hardy Rapids are important. Processes in italics are discussed only in this table]

\begin{tabular}{|c|c|}
\hline Change and process & Comments \\
\hline B. Outlet erosion & $\begin{array}{l}\text { B. At Le Hardy Rapids, very resistant threshold formed by } 1-3 \mathrm{~m} \text { ledge with interlocking micro- } \\
\text { spherulites near base of Lava Creek Tuff. Much more erodible units above and below. }\end{array}$ \\
\hline D. Tectonic stretching & D. Crustal thinning and downwarping above magma chamber and other ductile material. \\
\hline $\begin{array}{l}\text { E. Yellowstone Lake ceasing to } \\
\text { overflow }\end{array}$ & $\begin{array}{l}\text { E. A greater-than-likely drying of climate because loss from lake now about } 15 \text { percent by } \\
\text { evaporation and } 85 \text { percent by overflow. }\end{array}$ \\
\hline \multicolumn{2}{|r|}{ II. Increase in lake level } \\
\hline \multicolumn{2}{|r|}{ III. Possible increases or decreases in lake level } \\
\hline A. Faulting & $\begin{array}{l}\text { A. Faulting of appropriate magnitude and timing not apparent. Overall sag of shorelines and offset } \\
\text { on Fishing Bridge fault noted in outlet area. }\end{array}$ \\
\hline B. Glacial-isostatic rebound & $\begin{array}{l}\text { B. Glacial load nearly uniform over Yellowstone Lake basin producing even uplift. Rebound fast and } \\
\text { mostly during deglaciation because brittle-ductile transition is shallow in caldera. }\end{array}$ \\
\hline \multicolumn{2}{|r|}{ IV. Oscillations in lake level } \\
\hline $\begin{array}{l}\text { A. Geothermal inflation and } \\
\text { deflation }\end{array}$ & $\begin{array}{l}\text { A. Geothermal sealing, pressure buildup, and inflation followed by rupture of seal, release of } \\
\text { geothermal fluids, and deflation. A cycle produces no net change in lake level consistent with } \\
\text { paleoshorelines not rising toward caldera center. }\end{array}$ \\
\hline
\end{tabular}


half of this record, and this oscillation is the primary cause of lake level changes during the second half of this record (fig. 4). We suggest that this millennial-scale oscillation in lake levels, in part, reflects caldera-wide inflation and deflation similar to that recognized from releveling surveys and radar-interferometry studies. Our radiocarbon and archeologically dated history (fig. 4) provides a temporal scale two orders of magnitude longer than the historic studies. New observations of faulting and local shoreline deformation are based on LIDAR data. In addition to investigating the processes responsible for lake-level change, the history itself provides a framework for archeology, including possible human habitation of both subaerial and now-submerged shorelines.

A rising water level greatly enhances landward erosion and the development of wave-cut shorelines, a process described by the Bruun rule (Bruun, 1988; Schwartz, 1987). The better developed shorelines (S1 to S6 and higher) recognized by Meyer and Locke (1986) and Locke and Meyer (1994) appear to have been devel-
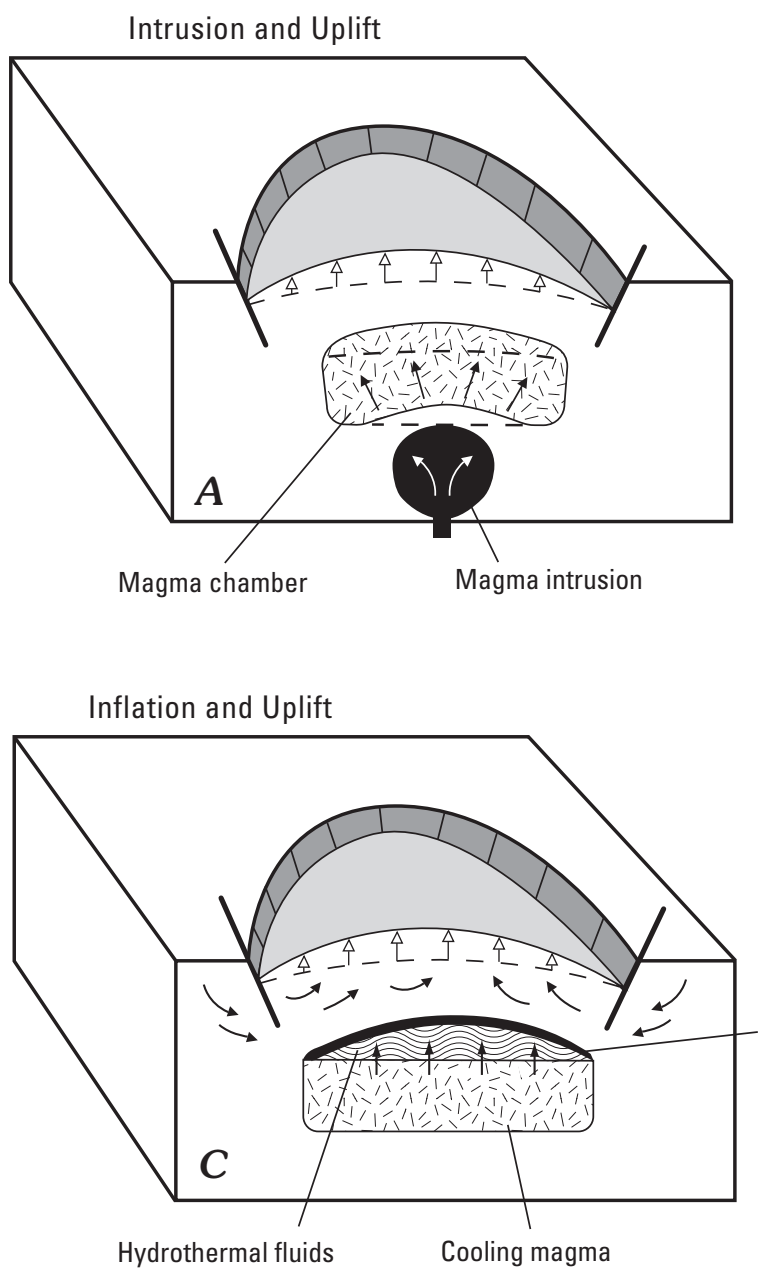

oped under rising lake conditions, as suggested by the Bruun rule and as discussed for the history of each shoreline in this report.

\section{Decreases in Lake Level}

\section{Holocene Outlet Erosion and Pleistocene Glacial Damming (I-A and 1-B in Table 4)}

Above $20 \mathrm{~m}$ at LHR, the Yellowstone River appears to have been downcutting mostly through unconsolidated glacial and alluvial material. Farther north in Hayden Valley, Richmond (1977) maps ice-dammed lake sediments of Pinedale age (pkl) to $2,375 \mathrm{~m}$ and locally to $2,390 \mathrm{~m}$ ( 20 m locally to $33 \mathrm{~m}$ above datum; LHR is $\sim 1 \mathrm{~m}$ below datum). Thus Yellowstone Lake levels from $\sim 20 \mathrm{~m}$ above datum and higher, or above S6, may have been dammed by recessional glaciers from the

\section{Extension and Subsidence}

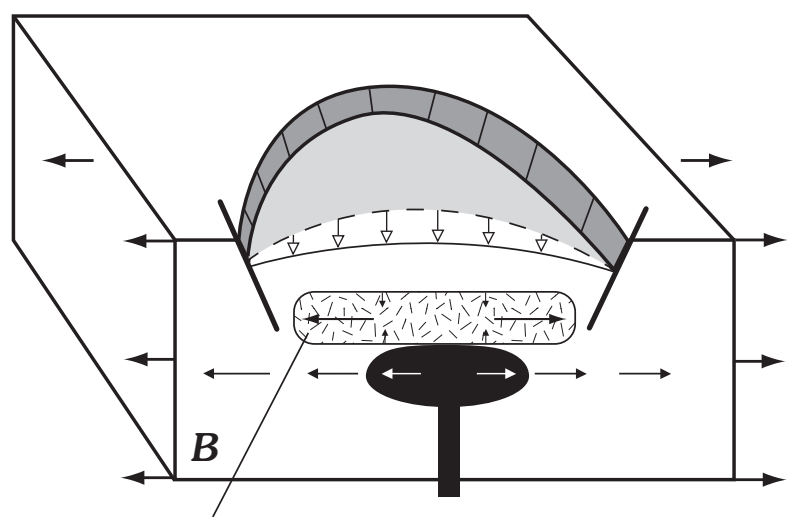

Thinned plastic body magma chamber

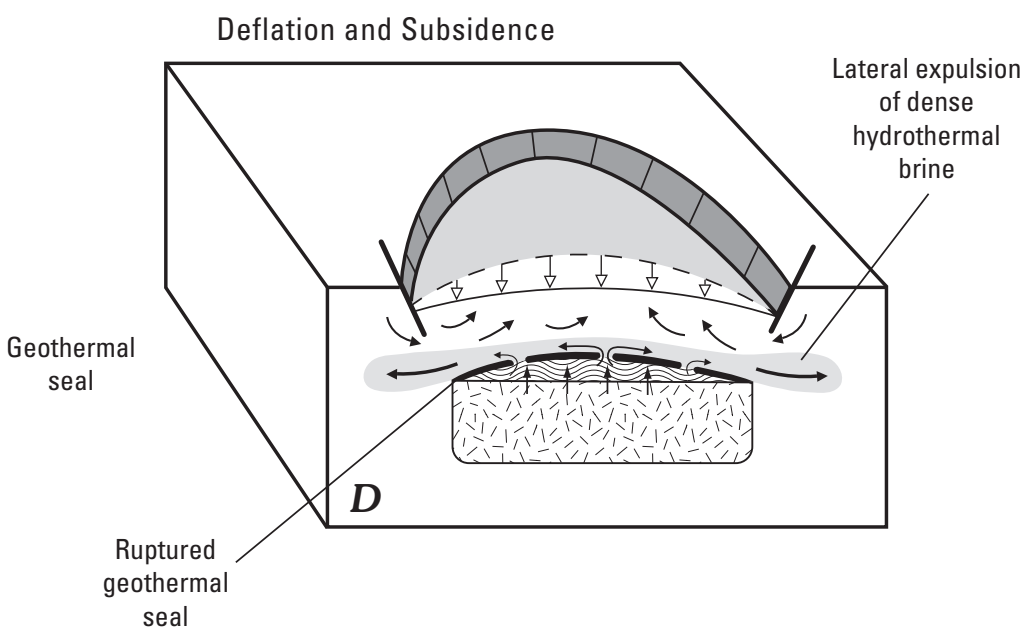

Figure 16. Diagrams showing mechanisms for inflation and deflation of Yellowstone caldera. Only some mechanisms are diagrammed. (A) Intrusion of magma and uplift. (B) Extension of crust and contained magma body resulting in subsidence above magma body. (C) Geothermal pressure buildup below seal and uplift. (D) Geothermal pressure release with rupture of seal and deflation accompanied by deep outward movement of dense fluids. Diagrams C and D are after Fournier (written commun., 1997). 
Beartooth uplift during Deckard Flats and younger times (Pierce, 1979).

Figure 4 shows a pattern of oscillating lake levels with a net lowering from $\sim 20 \mathrm{~m}$ above datum for S6 at $\sim 14.4 \mathrm{ka}$ to about the present lake level at S-meander time ( 10 ka). Assuming LHR has returned to the same vertical position for each shoreline, erosion of LHR at $\sim 5 \mathrm{~m} / \mathrm{k} . \mathrm{y}$. is indicated from 14.4 to $10 \mathrm{ka}$. Conversely, the change in shoreline levels may have been accompanied either by (1) tectonic sagging of LHR, in which case the rate of erosion would be less, or (2) net uplift of LHR, in which case the rate of erosion would be greater. Our correlations of shorelines from Fishing Bridge to the Mary Bay area are based on new LIDAR data and indicate a pronounced sagging; notably, S5 sags from $\sim 18 \mathrm{~m}$ to $\sim 10 \mathrm{~m}$ above datum from Mary Bay to the outlet.

Figure 4 also shows that, after $10 \mathrm{ka}$, lake levels have oscillated but with no clear trend. Hamilton and Bailey (1990, their fig. 3) draw a bedrock threshold at LHR $5 \mathrm{~m}$ above datum. Bedrock is locally mapped to $20 \mathrm{~m}$ above datum on the west side of LHR (Christiansen and Blank, 1975). An erosionally resistant zone near the base of the Lava Creek Tuff forms the bedrock ledge of Le Hardys Rapids (R.L. Christiansen, oral commun., 2002). This little-fractured, 1- to 3-m-thick devitrified zone is composed largely of interlocking microspherulites 1-3 mm across, making it quite resistant to erosion. Above this spherulite zone is a devitrified zone with joints on a decimeter scale that is more readily eroded. Below the spherulite zone, the Yellowstone River will next encounter the basal vitrophyre that is also much more fractured and also will be more readily eroded. The resistant bedrock zone appears to have been base level for the Yellowstone River for a considerable time, perhaps all of Holocene time. The $\sim 3$-ka gravel channel of the outlet reach is now below the level of this bedrock threshold. The 9.7-ka S-meander channel is drowned at its downstream end and thus projects at or below this bedrock threshold, suggesting little erosion of LHR over this time interval. The shorelines also show general sagging toward the outlet area (fig. 12) and thus toward the central part of the caldera (fig. 1), however, so some of the low altitude of the $\sim 9.7-\mathrm{ka}$ S-meander channel is probably from this sagging. The erosion rate of the bedrock threshold at LHR over the last 10 k.y. must be low in any case, as would be expected given the low bed-load transport rate at LHR. Bed-load supply in the outlet reach is limited to sand and fine gravel supplied by bank erosion and material carried to the outlet by longshore wave transport.

\section{Tectonic Stretching (I-D in Table 4)}

A crystallizing batholith probably exists beneath Yellowstone (see Christiansen, 2001, and references therein). Yellowstone is along the east margin of the Basin-and-Range structural province where tectonic extension is occurring. In addition, the Elephant Back fault zone offsets the 150-ka Elephant Back flow (fig. 5) indicating either significant SE-NW extension perpendicular to the caldera axis or keystone faulting associated with resurgent doming. Given that ductile material is as shallow as $4-5 \mathrm{~km}$ beneath the caldera (Smith and Bruhn, 1984; Fournier and Pitt, 1985; Smith and Braille, 1993), tectonic extension would stretch and thin the ductile body and cause subsidence perhaps accompanied by fissuring and faulting in the brittle material above (see fig. 16B). LHR lies along the axis of the caldera where the greatest subsidence due to extension might be expected. (See comment under "tectonic compression" regarding subsidence associated with convergence.)

\section{Yellowstone Lake Ceasing to Overflow (I-E in Table 4)}

Hamilton and Bailey (1988) observed submerged shorelines and suggested that they might indicate that Yellowstone Lake ceased to overflow. Locke and Meyer (1994) considered that Holocene climatic change was unlikely to have caused closed-basin conditions because an average of 45 percent of annual precipitation in the Yellowstone Lake basin currently discharges at the lake outlet. Studies of oxygen isotopes of water entering and exiting Yellowstone Lake suggest that about 15 percent of the inflow evaporates from the lake surface at present (Pat Shanks, oral commun., 2000). For Yellowstone Lake to cease overflowing, the ratio of evaporation to inflow would have to increase by more than six times, necessitating a very major climatic change. Pollen records from the Yellowstone Lake basin do not show evidence for dramatic late Holocene vegetation and climatic changes (Whitlock, 1993; Baker, 1976; Waddington and Wright, 1974). The simplest argument against closed-basin conditions is the drowned outlet channel of the Yellowstone River, which now lies well below the level of the bedrock threshold farther downstream at LHR. This channel was cut 4-3 ka when Yellowstone Lake was more than $5 \mathrm{~m}$ below its present level, yet, the lake was clearly overflowing and transporting gravel and undercutting its banks (fig. 6).

\section{Increases in Lake Level}

\section{Inflation by Magma Intrusion (II-A in Table 4)}

Decompression melting in the mantle from the Yellowstone hotspot source is thought to generate basaltic magma that rises upward to the base of the rhyolitic magma chamber (fig. 16A). Uplift or inflation because of magma intrusion is quite plausible (Pelton and Smith, 1982; Dzurisin and others, 1994). Modeling indicates that the historic inflation is consistent with a volume expansion involving either magmatic or hydrothermal fluids, primarily in the 3- to 6-km depth range (Vasco and others, 1990), or 8-km depth (Wicks and others, 1998). Uplift may relate either directly to this basaltic magma influx or to a rhyolitic magma generated by its heat and intruded at shallow levels within the caldera. 


\section{Tectonic Compression (II-D in Table 4)}

Local compression of a ductile magma chamber would produce uplift (Meertens and Levine, 1985). GPS studies show that radial contraction was accompanied by subsidence, suggesting the opposite effect (Smith and others, 1997; Meertens and others, 2000). This pattern (contraction and subsidence) suggests that subsidence is caused primarily by depressurization of a subcaldera source rather than by tectonic stretching.

\section{Possible Increases or Decreases in Lake Level}

\section{Faulting and Local Warping (III-A in Table 4)}

A north-trending fault system includes the Lake Hotel fault on the lake floor and the Fishing Bridge fault (see section on faulting discussed earlier). Although the Fishing Bridge fault offsets shoreline S5 and the wave-cut shoreline of S4 by several meters, it offsets the $\sim 9.7$-ka S-meander and surfaces that predate the $\mathrm{S}$-meander by less than $2 \mathrm{~m}$. The shorelines show an overall sag across the outlet area (fig. 12). The S2 shoreline is dated at $\sim 8 \mathrm{ka}$ on both sides of the Fishing Bridge fault and is only offset $0.5 \mathrm{~m}$ by the fault (fig. 14; table 3).

For the Lake Hotel graben, Johnson and others (2003) recognize $7.5 \mathrm{~m}$ of total offset on the western fault and 3.4 $\mathrm{m}$ of offset on the eastern antithetic fault; this results in a net offset of $4.1 \mathrm{~m}$. The lake floor is offset $2.7 \mathrm{~m}$ across the Lake Hotel graben (only $1.4 \mathrm{~m}$ less than the net offset of $4.1 \mathrm{~m}$ ). Based on sedimentation rates (Johnson and others, 2003), the last and by far the largest offset was $5.7 \mathrm{~m}$ on the western strand with a net offset of $2.9 \mathrm{~m}$ within the last $2 \mathrm{k} . \mathrm{y}$. We infer that such faulting dies out northward because the 8-ka S2 shoreline is essentially horizontal (fig. 12) and is offset only $0.5 \mathrm{~m}$ by the Fishing Bridge fault.

Tiller (1995) suggests that faulting might be responsible for increases in lake and river levels. Except for the Fishing Bridge fault, no faults with offset of shorelines have been recognized in the outlet area, including shorelines shown on bedrock and surficial geologic maps (Christiansen and Blank, 1975; Richmond, 1977) and those shorelines studied by Meyer and Locke (1986), Locke and Meyer (1994), Hamilton and Bailey (1990), and Pierce and others (1997). Just east of Le Hardy Rapids, John Good and Ken Pierce found a fault scarp with $\sim 1 \mathrm{~m}$ of down-to-the-northwest offset (fig. 5), which is opposite to that needed to raise the level of the outlet reach. The present drowned channel from the outlet to LHR does not change its nearly flat gradient or wide width as it might if a young fault crossed and uplifted the river channel (fig. 5). The submerged shoreline deposits at Bridge Bay and at Little Thumb Creek North, as well as the many drowned valleys around the lake, are not within any recognized graben, and thus local faulting did not cause their submergence.

Based on isostatic considerations and observed altitude changes after normal faulting, extensional faulting and associated crustal thinning is expected to produce 80 percent of the subsidence of the downthrown block and only 20 percent of the uplift of the upthrown block (Barrientos and others, 1987). Because the brittle-ductile transition zone in the caldera is only 20-35 percent as deep as normal in the Basin-and-Range, extensional structures arising from horizontal extension may have less vertical structural relief and may express extension through fissures and grabens.

\section{Glacial-Isostatic Rebound (III-B in Table 4)}

At the last glacial maximum, the ice cap on the Yellowstone Plateau reached an altitude greater than $3,350 \mathrm{~m}$ above Yellowstone Lake (altitude 2,358m) (Good and Pierce, 1998). The ice cap above the present Yellowstone Lake was about 1 $\mathrm{km}$ thick as demonstrated by flow from the lake area northward to over the top of Mount Washburn (altitude 3,125 m; Pierce, 1979) as well as flow eastward through Sylvan Pass at such high levels that it backfilled high into the valley of Middle Creek (Richmond and Pierce, 1972). With total compensation and an upper crust density of $2.7 \mathrm{gm} / \mathrm{cm}^{3}$, isostatic depression from 1 -km-thick ice (density $\sim 0.9 \mathrm{gm} / \mathrm{cm}^{3}$ ) would be $330 \mathrm{~m}$. Because the lithosphere is likely to be warm and more ductile beneath Yellowstone, a relatively high fraction of total potential compensation might have occurred close to the center of the load. In addition, because of this ductile material, the rate of rebound may be much greater than in continental craton areas. The thickness and load of ice above the Yellowstone Lake basin was relatively uniform (Good and Pierce, 1996), and thus near-uniform rebound is expected in the lake area, although cooler and stiffer crust is expected outside the caldera. Because both inflation and deflation have taken place during historic time as well as postglacial time (fig. 4), differential isostatic rebound is an unlikely mechanism to explain the historic shoreline record. This view is shared by Pelton and Smith (1982) and Locke and Meyer (1994).

This unweighting during deglaciation of about $1 \mathrm{~km}$ of ice (pressure $\sim 90$ bars) provides a natural experiment for evaluating the hazard of volcanic eruptions. During deglaciation, pressures in magmas beneath the Yellowstone Plateau were not high enough that the 90-bar reduction in confining pressure resulted in an eruption. In other areas-such as Hawaii (Porter, 1979), Iceland, and the "tuyas" of British Columbia—volcanic eruptions occurred through glacial ice that was melting and unloading during deglaciation. Thus, the pressure in the magmatic system beneath Yellowstone was not great enough to produce a volcanic eruption when rapid deglaciation about 14 ka reduced confining pressure on the system by 90 bars.

\section{Oscillations in Lake Level}

Figure 4 shows oscillations in lake level that can be divided into two parts: (1) an oscillating lowering to the S4 level >14 ka to $\sim 10.7 \mathrm{ka}$ and (2) oscillations near the present level from 10.7 ka to the present. The oscillating part of this pattern may be an 
Table 5. Change in altitude of $S 2, S 4$, and $S 5$ from near the caldera axis in the outlet to sites farther away, but in the caldera, to sites outside the caldera.

[Under the "Site" headings, first number is the shoreline altitude (in meters) above datum, and the second number is the distance from the caldera axis of historic uplift (in kilometers) (see fig. 1). For the outlet area, altitudes are for the eastern part of the Fishing Bridge peninsula]

\begin{tabular}{ccccc}
\hline Shoreline and age & $\begin{array}{c}\text { Site in outlet } \\
\text { area }\end{array}$ & $\begin{array}{c}\text { Site in caldera } \\
\text { but farther from axis }\end{array}$ & Site outside caldera & $\begin{array}{c}\text { Vertical decrease in height toward } \\
\text { caldera axis over distance }\end{array}$ \\
\hline$S 2$ & $5 \mathrm{~m} / 4 \mathrm{~km}$ & No site dated & $\begin{array}{c}7-8 \mathrm{~m} / 35 \mathrm{~km} \\
\text { delta in SE. arm }\end{array}$ & $2-3 \mathrm{~m} / 30 \mathrm{~km}$ \\
$8.0 \mathrm{ka}$ & $8-9 \mathrm{~m} / 4 \mathrm{~km}$ & $7 \mathrm{~m} / 17 \mathrm{~km}$ & No site dated & -1 to $-2 \mathrm{~m} / 13 \mathrm{~km}$, \\
increases toward caldera axis
\end{tabular}

* S5 uses height above datum as shown in figure 10 to Mary Bay crater wall, where $S 5$ is the same as $S 7$ of Locke and Meyer (1994, their fig. 4) and continues the same as $S 7$ of Locke and Meyer (1994) along the east shore of Yellowstone Lake.

extension of the observed 1923-1999 pattern of inflation and deflation based on releveling and radar-interferometry studies. The time intervals and amplitudes of inferred Holocene cycles are much greater, although rates are compatible.

The increase in lake level that defines the oscillations to the S5 and S4 shorelines is based on truncation of shorelines and valleys shown in figures 5 and 10. The amplitude is not known, but is estimated to be at least several meters. The increase in lake level both after the S-meander and after the 4- to 3-ka low is estimated to be $8.3 \mathrm{~m}$ between LHR and the outlet. Extending farther from the caldera axis, our studies of lake-level changes suggest differential uplift of $\sim 10-11 \mathrm{~m}$ at localities that span 41-43 percent of the 1923-1976 doming (table 2); the average long-term rate of uplift from 4-3 ka to the present is in the 0.7 to $1.2 \mathrm{~cm} / \mathrm{yr}$ range if extrapolated to the caldera margin at the outer limit of the 1923-1975 doming.

The oscillations suggesting rises near $5 \mathrm{ka}$ and $2 \mathrm{ka}$ (fig. 4) are not well established and are included as options because of evidence for a lake level near that of the present-day at about 4 and $2 \mathrm{ka}$. These oscillations suggest more rapid rates of uplift and subsidence between the outlet and LHR, and we are reticent to suggest that such rates are established (note slopes of uplift and subsidence on fig. 4). From 1923-1985, uplift of LHR relative to the outlet was $0.25 \mathrm{~m}$ at a rate of $4 \mathrm{~mm} / \mathrm{yr}$; to produce $8 \mathrm{~m}$ of uplift at this rate would take $2 \mathrm{k} . \mathrm{y}$.

Many of the prominent erosional shorelines, especially the ones designated S1-S5, were formed during rising lake levels and were most likely produced by an inflation episode. Locke and Meyer (1994) inferred that rising water levels resulting from the current episode of inflation (including inflation before the historic measurement period) were probably effective in producing the prominent modern wave-cut shorelines noted around Yellowstone Lake. This process probably accounts for the major past shorelines as well.

Unrest of calderas is common (Newhall and Dzurisin, 1988). The Phlegraean Fields caldera, Italy, a smaller trachytic caldera about $15 \mathrm{~km}$ across, has a 5,500-year record useful for comparison of rates, amplitudes, and patterns of inflation and deflation relative to Yellowstone (Dvorak and Mastrolorenzo, 1991). For the Phlegraean Fields caldera, Grindley (1976) charts $55 \mathrm{~m}$ of uplift from about 3,500 to 500 B.C. at about 18 $\mathrm{mm} / \mathrm{yr}$, followed by $15 \mathrm{~m}$ of subsidence from about 500 B.C. to about A.D. 1000 at a rate of $11 \mathrm{~mm} / \mathrm{yr}$. Subsequently, about $12 \mathrm{~m}$ of uplift led to an eruption in A.D.1528 followed by about $8 \mathrm{~m}$ of subsidence (A. Parascandola, cited in Yokoyama, 1971). These values are comparable to the historical values of uplift ( $15 \mathrm{~mm} / \mathrm{yr}$ ) and subsidence $(20-30 \mathrm{~mm} / \mathrm{yr}$ ) for the center of the Yellowstone caldera.

If the 1923-1985 caldera inflation at $15 \mathrm{~mm} / \mathrm{yr}$ were to continue for 1,000 to 4,000 years, uplift would total 15 to $60 \mathrm{~m}$. The subaerial shorelines do not have deformation of this magnitude, and the tilts are generally toward the caldera axis rather than away from it (fig. 12) (Locke and Meyer, 1994). Table 5 shows a compilation of overall apparent tilts with distance from the caldera axis for three shorelines. We selected S2 and S4 because they are independently dated over long distances. We selected S5 because this shoreline feature has been nearly continuously mapped (fig. 12) (Locke and Meyer, 1994). S5 of this paper along the northern lakeshore is the same as S7 of Locke and Meyer (1994) on the hydrothermal-explosion-crater rim above Beach Springs. S5 shows a significant tilt toward the caldera axis, and most of this occurs outside the caldera (table 5). S2 shows a 2- to 3-m tilt within the caldera toward the axis, whereas $\mathrm{S} 4$ shows a 1- to 2-m tilt away from the axis. In summary, net postglacial tilting of two of three shorelines shows gentle subsidence toward the axis of the caldera, and one shoreline shows a slight increase within the limits of measurement and dating. One explanation for the gentle tilts is that the prominent subaerial shorelines were formed at the culmination of an inflation cycle, and they are near-horizontal at present because the present shoreline may also be forming at the culmination of an inflation cycle. This has returned the older shorelines to approximately their original horizontal positions. The cumulative gentle overall subsidence toward the caldera 
axis might be due to magmatic cooling. Fournier and Pitt (1985) estimate subsidence of $0.6-0.7 \mathrm{~mm} / \mathrm{yr}$ due to cooling; over the age of S5 (14.6 ka), this would total $7.5 \mathrm{~m}$, which is within the range of estimates of subsidence (table 5).

\section{Two Favored Explanations for Record of Oscillations}

A nearly steady state process with little net volume change in postglacial time within the caldera seems to be required to produce the observed deformation history (table 5). Inflation and deflation of the caldera in the last 100 years has been called "breathing," we use the term "heavy breathing" for millennial-scale, larger episodes of inflation and deflation. This "heavy breathing" of the central part of the Yellowstone caldera may reflect magmatic inflation and tectonic stretching and deflation, or (our preferred interpretation) hydrothermal-fluid sealing and inflation followed by cracking and deflation (fig. 16). The other factors noted in table 4 may also be involved. Listed below are two explanations, one involving only hydrothermal processes and the other a hybrid of volcanic and tectonic processes.

\section{Hydrothermal Inflation and Deflation (IV-A in Table 4)}

A large, magmatically driven hydrothermal system exists principally within the 640-ka Yellowstone caldera (Fournier and Pitt, 1985; Fournier, 1989; Christiansen, 2001). At a depth of about $5 \mathrm{~km}$, a seal may be created by mineral deposition and ductile flow, separating a deep zone associated with crystallizing magma and its expelled fluids and in which pore pressures can approach lithostatic, from a shallower zone, where pore pressures are hydrostatic (Fournier and Pitt, 1985; Fournier, 1989). Hydrothermal-pressure buildup of the confined hydrothermal fluids beneath this seal would produce uplift. Eventual rupture of the seal, perhaps during an earthquake or hydrofracturing, would permit these confined hydrothermal fluids to escape and result in subsidence, perhaps back to the original level. This mechanism could explain why even latest Pleistocene shorelines show little net deformation, with net inflation being equal to net deflation.

Fournier (1989) calculated that the volume of magmatic fluids released by magma crystallization is adequate to explain historic uplift rates. Fournier (oral commun., 2002) also estimates that no more than a 10-percent increase in surface manifestation of geothermal fluids has occurred, but that dense brines from beneath the ruptured seal might not be vented directly to the surface. Instead, the brines may be expelled laterally at depth. Friedman (this volume) finds that there has been a decrease in chloride representing hydrothermal heat output of about 10 percent since the early 1980s, about when subsidence started in 1985. Friedman also notes that years and seasons of increased precipitation also correlate with increased chloride release. We suggest that the 10-percent decrease noted by Friedman might reflect the general drought that has occurred since about 1980 (Despain, oral commun., 2002) and thus does not necessarily contradict the postulate of geothermal release producing the subsidence after 1985. Dzurisin and others (1994, p. 268) note a cluster of hydrothermal and earthquake events (Nagy and Smith, 1988; Waite and Smith, 2002) within and outside the caldera that may relate to the 1985 change from inflation to deflation and may be associated with rupture of a deep hydrothermal seal and release of hydrothermal fluids. In this regard, it is interesting to note that the banding of vein deposits, commonly noted in mineralized areas around intrusions, may also indicate pulsations in the outflow of hydrothermal fluids (Fournier, 1999).

A correlation between hydrothermal explosions and associated reduction of deep, confined hydrothermal pressures might also result in lowering of LHR and lake levels. Figure 4 shows that the ages of three hydrothermal explosions do not clearly correlate with times of lake-level lowering, although the dating of the explosions and lake level both have considerable uncertainty. Other explosions-such as Duck Pond, Fern Lake, the subaqueous Elliott's crater (age 13-8 ka; Johnson and others, 2003), and other craters-need to be dated to fully evaluate this possible relation.

\section{Inflation by Magma Intrusion and Subsidence by Other Mechanisms (IV-B in Table 4)}

Because Yellowstone is an active volcanic field, a magmatic explanation is obviously plausible (Pelton and Smith, 1982; Dzurisin and others, 1994). The inflation has been modeled with the largest volume expansions in the 3.0- to 6.0-km-depth range (Vasco and others, 1990). The overall horizontality of shorelines (table 5) indicates that there has been no net doming by buildup of postglacial intrusions. The subsidence part of the oscillation is more difficult to explain by evacuation of the emplaced magma, although the radially outward earthquake patterns may represent outward magma movements. Subsidence due to tectonic stretching of ductile material, primarily of the batholithic magma chamber, is predictable given Yellowstone's location on the margin of the extending Basin-and-Range. The Elephant Back fault system indicates SE-NW extension that may reflect either fracturing associated with localized doming along the caldera axis or regional extension; such doming does not provide an explanation for subsidence, but regional extension does. Outside of the Yellowstone caldera, extensional Basin-and-Range faults are clearly active, including the Teton fault, the east Sheridan fault, and the Hebgen earthquake faults. Such extension might be episodic and produce subsidence of the caldera. The Eagle Bay fault has been active in Holocene time and extends into the caldera from the south (fig. 2).

Subsidence due to cooling is almost certainly occurring and estimated to be $0.6-0.7 \mathrm{~mm} / \mathrm{yr}$ (Fournier and Pitt, 1985), but it can account for only a small fraction of the historically observed rates of about 15- to 30-mm/yr uplift and subsidence (Dzurisin and others, 1994, their fig. 4). This suggests that 
cooling is a minor factor in subsidence. If inflation is explained by magma intrusion and deflation is explained by extension or other processes, the following questions are of concern: (1) why would the volume of magma intrusion and associated uplift equal the volume of extension and subsidence?, and (2) why would these alternate systematically in time?

\section{Local Doming and Faulting of Shorelines}

In addition to the changes in lake level, remarkable local deformation is recorded by shoreline faulting and tilting. The cause of the tilting and doming is locally associated with hydrothermal centers. For example, west from the Storm Point hydrothermal center with its dramatic craters (fig. 10), uplift is $6 \mathrm{~m}$ over a kilometer (fig. 15). A localized anticline just east of Pelican Creek has no surface hydrothermal features, although hot beach sand and steamy unfrozen lake margin is noted along trend to the south and merits further investigation. On the lake floor, domal areas attributed to hydrothermal processes have even greater relief than these onshore sites (Johnson and others, 2003). Outside of the northern Yellowstone Lake study area, Locke and Meyer (1994) show broad uplift of the Rock Point area and subsidence of the West Thumb area.

The Fishing Bridge fault shows increasing offset of older shorelines. Tilting of shorelines into the fault is equal to or greater than fault offset. Over a distance of about 1.1 $\mathrm{km}$ across the Fishing Bridge peninsula, tilt of shorelines into the Fishing Bridge fault increase from $0.8 \mathrm{~m}$ for S2 to $6.7 \mathrm{~m}$ (projected) for S5 (fig. 14; table 3).

\section{Conclusions}

$\mathrm{S} 2$ (8 ka) and related shorelines are tilted as much as 6 $\mathrm{m} / \mathrm{km}$ away from the Storm Point hydrothermal center. Just west of this tilted area is a local dome more than $2 \mathrm{~m}$ high and about $0.5 \mathrm{~km}$ wide. Both of these uplifts suggest shallow emplacement of a volume, presumably of magma or hydrothermal fluids. This, and similar features on the lake floor and perhaps elsewhere, may represent a significant hazard.

LIDAR data permit recognition of the Fishing Bridge fault and its history of Holocene offset. Near Fishing Bridge, the fault offsets the $\mathrm{S} 2$ shoreline $0.5 \mathrm{~m}$ and the $\mathrm{S} 4$ barrier beach $1 \mathrm{~m}$. About $2 \mathrm{~m}$ of faulting occurred after eroding the wave-cut $\mathrm{S} 4$ but before building the S4 barrier beach. The Fishing Bridge fault, $0.5-1.5 \mathrm{~km}$ farther north, offsets by $1-2 \mathrm{~m}$ the $\mathrm{S} 2$ sand spit filling the $\mathrm{S}$-meander, but it offsets less than $2 \mathrm{~m}$ adjacent surfaces that predate the S-meander-indicating offset after $\sim 11 \mathrm{ka}$ (S4) is the same as that after $8 \mathrm{ka}(\mathrm{S} 2)$. Curiously, late Holocene activity on the Fishing Bridge fault has been minimal (table 2), whereas activity on the offshore Lake Hotel fault (Johnson and others, 2003) has been mostly late Holocene.

Shorelines S2 and S4 are correlated by independent dating from the north to south shores of the lake and are essentially horizontal, and, significantly, they do not rise toward the caldera axis. Our correlation of S5, a well-represented shoreline along the north and east side of the lake, gently slopes toward the caldera axis. This overall near-horizontality contrasts with local doming and faulting of shorelines where structural relief exceeds $5 \mathrm{~m}$.

We reconstruct the following postglacial history of changes for Yellowstone Lake and adjacent areas (fig. 4).

1. MB hydrothermal explosion occurred about $13 \mathrm{ka}$ and mantles S5.5 and S6, but not S5. The steep crater wall on the north side of Mary Bay was formed during the MB explosion, and S5 was cut into the crater wall.

2. Using LIDAR images, S5 can be traced from Pelican Creek to Mary Bay, where it is S6 of Meyer and Locke (1986) and S7 of Locke and Meyer (1994). S5 postdates the MB hydrothermal explosion and is $\sim 12.6 \mathrm{ka}$.

3. The $\mathrm{S} 4$ shoreline is $\sim 10.7 \mathrm{ka}$ and is $7-9 \mathrm{~m}$ above datum in the Fishing Bridge area and 7-8 $\mathrm{m}$ above datum east of the Grant Village area. Locke and Meyer (1994) note local uplift of S4 of about $4 \mathrm{~m}$ in the Mary Bay and Rock Point area to about $12 \mathrm{~m}$ above datum.

4. The S-meander represents a low level of Yellowstone Lake and LHR that occurred after the S4 shoreline and before S2 and S3 shorelines. Uplift of LHR by more than $8 \mathrm{~m}$ relative to the outlet converted this once vigorously flowing reach of the river to an arm of the lake.

5. The $\mathrm{S} 2$ shoreline is about $4-6 \mathrm{~m}$ above datum on both sides of the outlet and dates $\sim 8 \mathrm{ka}$ on both sides of the outlet, confirming little or no deformation.

6. Yellowstone Lake has been near or below its present level from after $\mathrm{S} 2$ time ( $8 \mathrm{ka})$ to present.

7. About 4-3 ka in Bridge Bay and in the drowned valley of Little Thumb Creek North, lake level was 5.4 and $6.3 \mathrm{~m}$ lower, respectively, than the present $\mathrm{S} 1$ shoreline. The outlet reach of the Yellowstone River is presently a drowned channel that had a much higher gradient before 4-3 ka. Estimation of original river gradients indicates uplift of LHR relative to the outlet was about $8 \mathrm{~m}$ and thus converted this reach from a once vigorously flowing river to the present "pool."

8. Other evidence of now-submerged lake-level features that is widespread but not yet dated includes (1) drowned valleys such as Pelican Creek, Sedge Creek, and Little Thumb Creek, (2) submerged shorelines noted by Johnson and others (2003) 2-4 m below datum (their $\mathrm{S}^{-1}$ ) and 10-13 m below datum (their $\mathrm{S}^{-3}$ ), and (3) shorelines submerged 15-30 m below datum noted by Hamilton and Bailey (1990) at many tens of locations. Better dating and correlation are needed to understand these submerged shorelines.

The overall pattern of lake level changes (fig. 4) suggests an oscillating lowering of lake level from at least the S6 level at 14.4 ka to about $\mathrm{S} 4$ time at $10.7 \mathrm{ka}$. This is easiest to explain by 
erosion of the outlet accompanied by inflation-deflation cycles; however, the tilt of S5 toward the caldera axis (table 5) may indicate 6-9 $\mathrm{m}$ of post-S5 subsidence of the threshold at LHR. After S4 time, lake oscillations predominate over outlet erosion. After S2 time ( 8 ka), the lake has been below or near its present level also indicating little outlet erosion. The increase in lake level from 4-3 ka to present (fig. 4) can be explained by uplift of LHR.

The cyclic model suggested by historic deflation and inflation explains many aspects of the record throughout the last $15 \mathrm{k} . \mathrm{y}$. In particular, it explains lake-wide net deformation of shorelines of generally less than $10 \mathrm{~m}$ in contrast to historical rates of uplift and subsidence of up to 1-2 cm/yr (10-20 m/1,000 yr). If shorelines were cut during intervals of rising (transgressing) lake level as suggested by Locke and Meyer (1994), the present lake level (S1) and shorelines S3, S4, and S5 probably represent lake-level culminations. This sequence of shorelines is subhorizontal or declines toward the caldera axis, apparently because they represent culminations of uplift in an oscillating pattern that has produced no net uplift. Because the present time appears to be a culmination, the other culminations may also appear essentially horizontal. This history and geometry support cycles of inflation and deflation with an amplitude of about $8 \mathrm{~m}$ between LHR and the outlet and a frequency of perhaps $1-3 \mathrm{k} . \mathrm{y}$.

For caldera inflation or deflation, several mechanisms are likely to be operating: magma intrusion, crustal stretching, batholithic cooling, and hydrothermal pressure changes. The mechanism of uplift by hydrothermal-pressure buildup beneath a hydrothermal seal, followed by subsidence due to rupture of the seal and release of fluids, is appealing because, by itself, it explains the present large-scale near-horizontality of shorelines. Local sagging of shorelines may be explained by extension and faulting, whereas local doming may result from hydrothermal or magmatic buildup. Subaerial shorelines that represent inflation culminations and relative lake-level highstands are subhorizontal. Submerged shorelines that formed during deflation episodes and overall lower lake levels might be expected to be more tilted than shown by Johnson and others (2003), but, as suggested by the Bruun rule, even the submerged shorelines might represent minor culminations of lake level during longer period oscillations. Because subaerial postglacial shorelines do not strongly slope away from the caldera axis, either voluminous magma intrusions have not accumulated or the uplift volume of such intrusions has been fortuitously balanced by subsidence processes such as tectonic stretching or batholithic cooling. Inflation appears responsible for the lake-level rises (fig. 4), but such inflation has not resulted in volcanic eruptions or cumulative doming of shorelines tilting away from the caldera axis. The inflation-deflation cycles seem to represent an essentially zero-sum process with little net subsurface volume change, which seems most readily explained by buildup and release of hydrothermal fluids.

Hydrothermal explosions are likely associated with lowering of pressure of the confined hydrothermal system and subsidence. Wicks and others (1998) conclude that transfer of fluids over tens of kilometers between the two resurgent domes has occurred in a few years, which would indicate that caldera-scale interconnections may occur on a similar time scale. The ages of three hydrothermal explosions do not clearly correlate with times of lake-level deflation (fig. 4), although the dating of the explosions and lake level both have considerable uncertainty. Other hydrothermal-explosion craters-such as Duck Lake, Fern Lake, the subaqueous Cutthroat Crater, and other craters-need to be dated to better evaluate this hypothesis.

The Yellowstone caldera was covered with about $1 \mathrm{~km}$ of glacial ice about 20-16 ka. Deglaciation and the reduction in pressure by about 90 bars was not accompanied by volcanic eruptions, suggesting the magmatic system was not then, and perhaps not now, at pressures high enough for eruption.

\section{Acknowledgments}

We appreciate the work of the crew of the Midwest Archeological Center in finding archeological and charcoal material for dating, and we particularly appreciate the surveying work of George Crothers. The Park Service staff at Lake Village was particularly helpful, including John Lounsbury, Dan Reinhart, Bruce Sefton, Loyd Kortge, Harlan Credit, Dan Mahony, and the NPS dive team. Kerry Cook and Dan Lofgren of the Federal Highways Administration provided records and samples from borings. We appreciate field conferences with Bob Christiansen, Pat Muffler, Rick Hutchinson, and Wayne Hamilton. Bob Fournier greatly aided our understanding of hydrothermal processes and their relation to uplift and subsidence processes. Bob Smith provided GPS data on the ongoing deformation. Dan Dzurisin provided surveying data and advice concerning deformation processes. Pierce particularly appreciates field work together and discussions with Lisa Morgan and Pat Shanks regarding the lake floor and hydrothermal explosions. Bill Locke did the definitive surveying of the shorelines and discussed his interpretations freely with us. Bill Locke and Dan Dzurisin provided very helpful reviews, and Tom Judkins provided thorough editing.

\section{References Cited}

Baker, R.G., 1976, Late Quaternary vegetation history of the Yellowstone Lake basin, Wyoming: U.S. Geological Survey Professional Paper 729-E, p. E1-E48.

Barrientos, S.E., Stein, R.S., and Ward, S.N., 1987, Comparison of the 1959 Hebgen Lake, Montana, and the 1983 Borah Peak, Idaho, earthquakes from geodetic observations: Bulletin of the Seismological Society of America, v. 77, p. 784-808. 
Bruun, P., 1988, The Bruun rule of erosion by sea-level riseA discussion on large-scale two- and three-dimensional usages: Journal of Coastal Resources, v. 4, p. 627-648.

Cannon, K.P., Crothers, G.M., and Pierce, K.L. 1994, Archeological investigations along the Fishing Bridge Peninsula, Yellowstone National Park, Wyoming-The archeology, geology and paleoenvironment: Lincoln, Nebr., National Park Service, Midwest Archeological Center Technical Report on file, $367 \mathrm{p}$.

Cannon, K.P., Pierce, K.L., and Crothers , G.M., 1995, Caldera unrest, lake levels, and archeology-The view from Yellowstone Lake: Park Science, v. 15, no. 3, p. 28-31.

Cannon, K.P., Pierce, L.L., Stormberg, P., and MacMillian, M.V., 1997, Results of archeological and paleoenvironmental investigations along the north shore of Yellowstone Lake, Yellowstone National Park, Wyoming-1990-1994: Lincoln, Nebr., National Park Service, Midwest Archeological Center Technical Report on file, 458 p.

Christiansen, R.L., 1984, Yellowstone magmatic evolution-Its bearing on understanding large-volume explosive volcanism, in Explosive volcanism-Inception, evolution, and hazards: Washington, D.C., National Academy Press, p. 84-95.

Christiansen, R.L., 2001, The Quaternary and Pliocene Yellowstone Plateau volcanic field of Wyoming, Idaho, and Montana: U.S. Geological Survey Professional Paper 729G, 145 p.

Christiansen, R.L., and Blank, H.R., Jr., 1975, Geologic map of the Canyon Village quadrangle, Yellowstone National Park, Wyoming: U.S. Geological Survey Geologic Quadrangle Map GQ-1192, scale 1:62,500.

Doerner, J.P., and Carrara, P.E., 2001, Late Quaternary vegetation and climatic history of the Long Valley area, west-central Idaho, U.S.A.: Quaternary Research, v. 56, p. 103-111.

Dvorak, J.J., and Mastrolorenzo, Giuseppe, 1991, The mechanisms of recent crustal movements in Campi Flegrei caldera, southern Italy: Geological Society of America Special Paper 263, $47 \mathrm{p}$.

Dzurisin, Daniel, Christiansen, R.L., and Pierce, K.L., 1995, Yellowstone-Restless volcanic giant: U.S. Geological Survey Open-File Report 95-59 (Fact Sheet), 2 p.

Dzurisin, Daniel, Savage, J.C., and Fournier, R.O., 1990, Recent crustal subsidence at Yellowstone caldera, Wyoming: Bulletin of Volcanology, v. 52, p. 247-270.

Dzurisin, Daniel, and Yamashita, K.M., 1987, Vertical surface displacements at Yellowstone caldera, Wyoming, 19761986: Journal of Geophysical Research, v. 92, no. B13, p. $13,753-13,766$.
Dzurisin, Daniel, Yamashita, K.M., and Kleiman, J.W., 1994, Mechanisms of crustal uplift and subsidence at the Yellowstone caldera, Wyoming: Bulletin of Volcanology, v. 56, p. 261-270.

Fournier, R.O., 1989, Geochemistry and dynamics of the Yellowstone National Park hydrothermal system: Annual Review Earth Planetary Science, v. 17, p. 13-53.

Fournier, R.O., 1999, Hydrothermal processes related to movement of fluid from plastic into brittle rock in the magmatic-epithermal environment: Economic Geology, v. 94, p. $1,193-1,212$.

Fournier, R.O., and Pitt, A.M., 1985, The Yellowstone magmatic-hydrothermal system, in Stone, Claudia, ed., International symposium on geothermal energy, international volume: Geothermal Resources Council Transactions, p. 319-327.

Frison, G.C., 1991, Prehistoric hunters of the High Plains (2d ed.): New York, Academic Press, 532 p.

Frison, G.C., 1992, The foothills-mountains and the open plains-The dichotomy in paleoindian subsistence strategies between two ecosystems, in Stanford, D.J., and Day, J.S., eds., Ice Age hunters of the Rockies: Niwot, Colo., University Press of Colorado, p. 323-362.

Good, J.D., and Pierce, K.L., 1996, Interpreting the landscapes of Grand Teton and Yellowstone National Parks, recent and ongoing geology: Grand Teton National History Association, 58 p., 57 illus., $2 \mathrm{~d}$ printing, 1998.

Greiser, S.T., 1986, Artifact collections from ten sites at Canyon Ferry Reservoir: Archeology in Montana, v. 27, no. 1-2, p. 1-190.

Grindley, G.W., 1976, Relation of volcanism to Earth movements, Bay of Naples, Italy, in Gonzales-Ferran, O., ed., Proceedings symposium on Andean and Antarctic volcanology problems: Rome, IAVEC, p. 598-612.

Hamilton, W.L., 1987, Water level records used to evaluate deformation within the Yellowstone caldera, Yellowstone National Park: Journal of Volcanology and Geothermal Research, v. 31, p. 205-215.

Hamilton, W.L., and Bailey, A.L., 1988, Holocene deformation deduced from shorelines at Yellowstone Lake, Yellowstone caldera, I: Historical summary and implications:Eos, Transactions of the American Geophysical Union, v. 69, no. 44, p. 1,472 .

Hamilton, W.L., and Bailey, A.L., 1990, Holocene deformation history of the Yellowstone caldera at Yellowstone Lake inferred from abandoned shorelines and sediments: Yellowstone Physical Sciences Laboratory, Yellowstone National Park, Report 90-3, 24p., 13 figs. [draft of 8 June, 1990]. 
Husted, W.M., and Edgar, R., 2002, The archeology of Mummy Cave, Wyoming-An introduction to Shoshonean prehistory: Lincoln, Nebr., National Park Service, Midwest Archeological Center Special Report 4 and Southeast Archeological Center Technical Report Series 9, 231 p.

Johnson, S.Y., Stephenson, W.J., Morgan, L.A., Shanks, W.C., III, and Pierce, K.L., 2003, Postglacial geologic processes in northern Yellowstone Lake, Wyoming: Geological Society of America Bulletin, v. 115, no. 8 p. 954-971.

Kaplinski, M.A., 1991, Geomorphology and geology of Yellowstone Lake, Yellowstone National Park, Wyoming: Flagstaff, Northern Arizona University, M.S. thesis, 82 p.

Licciardi, J.M., Clark, P.U., Brook, E.J., Pierce, K.L., Kurz, M.D., Elmore, D., and Sharma, P., 2001, Cosmogenic ${ }^{3} \mathrm{He}$ and ${ }^{10} \mathrm{Be}$ chronologies of the northern outlet glacier of the Yellowstone ice cap, Montana, USA: Geology, v. 29, p. 1,095-1,098.

Locke, W.W., 1986, Shoreline elevation data for the roaded portion of Yellowstone Lake shore, in Locke, W.W., unnamed editor, Quaternary evolution of the Yellowstone region: Friends of the Pleistocene, Rocky Mountain Cell, Guidebook, p. 52-53.

Locke, W.W., and Meyer, G.A., 1994, A 12,000-year record of vertical deformation across the Yellowstone caldera margin-The shorelines of Yellowstone Lake: Journal of Geophysical Research, v. 99, no. B10, p. 20,079-20,094.

Locke, W.W., Meyer, G.A., and Pings, J.C., 1992, Morphology of a post-glacial fault scarp across the Yellowstone (Wyoming) caldera margin and its implications: Bulletin of the Seismological Society of America, v. 82, p. 511-516.

Machette, M.N., Pierce, K.L., McCalpin, J.P., Haller, K.M., and Dart, R.L., 2001, Map and data for Quaternary faults and folds in Wyoming: U.S. Geological Survey Open-File Report 01-461, 155 p., map.

Mehringer, P.J., Jr., Sheppard, J.C., and Foit, F.F., Jr., 1984, The age of the Glacier Peak tephra in west-central Montana: Quaternary Research, v. 21, p. 36-41.

Meertens, C.M., and Levine, J., 1985, Compressive tectonic strain as a possible mechanism for long-term vertical deformation of the Yellowstone caldera: Eos, Transactions of the American Geophysical Union, v. 66, no. 46, p. 853.

Meertens, C.M., and Smith, R.B., 1991, Crustal deformation of the Yellowstone caldera from first GPS measurements-1987-1989: Geophysical Research Letters, v. 18, p. $1,763-1,766$.
Meertens, C.M., Smith, R.B., and Puskas, C.M., 2000, Crustal deformation of the Yellowstone caldera from campaign and continuous GPS surveys, 1987-2000 [abs.]: Eos, Transactions of the American Geophysical Union, v. 78, no. 46, p. V22F-19.

Meyer, G.A., 1986, Morphology of the Yellowstone River below the nominal lake outlet at Fishing Bridge and topographic map of the Lake Lodge area, in Locke, W.W., unnamed editor, Quaternary evolution of the Yellowstone region: Friends of the Pleistocene, Rocky Mountain Cell, Guidebook, p. 47-49.

Meyer, G.A., and Locke, W.W., 1986, Origin and deformation of Holocene shoreline terraces, Yellowstone Lake, Wyoming: Geology, v. 14, p. 699-702.

Morgan, L.A., and Shanks, W.C., 2005, Influences of rhyolitic lava flows on hydrothermal processes in Yellowstone Lake and on the Yellowstone Plateau, in Inskeep, W.P., and McDermott, T.R., eds., Geothermal biology and geochemistry in Yellowstone National Park: Proceeding of the Thermal Biology Institute workshop, Yellowstone National Park, Wyoming, p. 53-72.

Morgan, L.A., Shanks, W.C., III, Lovalvo, D.A., Johnson, W.J., Pierce, K.L., Harlan, S., Finn, C.A., Lee, G., Webring, M., Schulze, B., Duhn, J., Sweeney, R., and Balistrieri, L., 2003, Exploration and discovery in Yellowstone LakeResults form high-resolution sonar imaging, seismic reflection profiling, and submersible studies: Journal of Volcanology and Geothermal Research, v. 122, p. 221-242.

Nagy, W.C., and Smith, R.B., 1988, Seismotectonic implications of the 1985-86 Yellowstone earthquake swarm from velocity and stress inversion: Seismological Research Letters, v. 59, p. 20.

Newhall, C.G., and Dzurisin, Daniel, 1988, Historic unrest at large calderas of the world: U.S. Geological Survey Bulletin $1855,1,108$ p., 2 vols.

Otis, R.R., Smith, R.B., and Wold, R.J., 1977, Geophysical surveys of Yellowstone Lake, Wyoming: Journal of Geophysical Research, v. 82, p. 3,705-3,717.

Pelton, J.R., and Smith, R.B., 1979, Recent crustal uplift in Yellowstone National Park: Science, v. 206, p. 1,179-1,182.

Pelton, J.R., and Smith, R.B., 1982, Contemporary vertical surface displacements in Yellowstone National Park: Journal of Geophysical Research, v. 87, no. B4, p. 2,745-2,761.

Pierce, K.L., 1979, History and dynamics of glaciation in the northern Yellowstone National Park area: U.S. Geological Survey Professional Paper 729-F, 91 p. 
Pierce, K.L., 2003, Pleistocene glaciations of the Rocky Mountains, in Gillespie, A.R., Porter, S.C., and Atwater, B.F., eds., The Quaternary Period in the United StatesDevelopments in Quaternary Science, v. 1: Amsterdam, Elsevier, p. 63-76.

Pierce, K.L., Cannon, K.P., and Crothers, G.M., 1994, Archeological implications of changing lake levels of Yellowstone Lake, Yellowstone National Park, Wyoming: Current Research in the Pleistocene, v. 11, p. 106-108.

Pierce, K.L., Cannon, K.P., and Meyer, G.A., 1997, Yellowstone caldera "heavy breathing" based on Yellowstone Lake and River changes in post-glacial time: Eos, Transactions of the American Geophysical Union, v. 78, no. 46, p. F802.

Pierce, K.L., Cannon, K.P., Meyer, G.A., Trebesch, M.J., and Watts, Raymond, 2002, Post-glacial inflation-deflation cycles, tilting, and faulting in the Yellowstone caldera based on Yellowstone Lake shorelines: U.S. Geological Survey Open-File Report 02-0142, 30 p., 16 figs., 5 tables.

Porter, S.C., 1979, Hawaiian glacial ages: Quaternary Research, v. 12, p. 161-187.

Porter, S.C., Pierce, K.L., and Hamilton, T.D., 1983, Late Pleistocene glaciation in the Western United States, in Porter, S.C., ed., The late Pleistocene, v. 1, of Wright, H.E., Jr., ed., Late Quaternary environments of the United States: Minneapolis, Minn., University of Minnesota Press, p. $71-111$.

Reeve, S.A., 1989, Prehistoric settlements at the Yellowstone Lake outlet, Yellowstone National Park, Wyoming: Lincoln, Nebr., National Park Service, Midwest Archeological Center Technical Report on file, $161 \mathrm{p}$.

Richmond, G.M., 1973, Surficial geologic map of the West Thumb quadrangle, Yellowstone National Park, Wyoming: U.S. Geological Survey Miscellaneous Investigations Series Map I-643, scale 1:62,500.

Richmond, G.M., 1974, Surficial geologic map of the Frank Island quadrangle, Yellowstone National Park, Wyoming: U.S. Geological Survey Miscellaneous Investigations Series Map I-642, scale 1:62,500.

Richmond, G.M., 1976, Surficial geologic history of the Canyon Village quadrangle, Yellowstone National Park, Wyoming, for use with Map I-652: U.S. Geological Survey Bulletin 1427, 35 p.

Richmond, G.M., 1977, Surficial geologic map of the Canyon Village quadrangle, Yellowstone National Park, Wyoming: U.S. Geological Survey Miscellaneous Investigations Series Map I-652, scale 1:62,500.
Richmond, G.M., and Pierce, K.L., 1972, Surficial geologic map of the Eagle Peak quadrangle, Yellowstone National Park and adjoining area, Wyoming: U.S. Geological Survey Miscellaneous Investigations Series Map I-637, scale $1: 62,500$.

Schwartz, M.L., 1987, The Bruun rule-twenty years later: Journal of Coastal Resources, v. 3, p. ii-iv.

Shortt, M. W., 2001, The Osprey Beach site-A Cody Complex occupation on the south shore of West Thumb [abs.]: 6th Biennial Scientific Conference on the Greater Yellowstone Ecosystem, abstracts, p. 37.

Smith, R.B., and Braile, L.W., 1993, Topographic signature, space-time evolution, and physical properties of the Yellowstone-Snake River Plain volcanic system-The Yellowstone hotspot, in Snoke, A.W., Steidtmann, J.R., and Roberts, S.M., eds., Geology of Wyoming: Geological Survey of Wyoming Memoir 5, p. 694-754.

Smith, R.B., and Bruhn, R.L., 1984, Intraplate extensional tectonics of the western U.S. Cordillera-Inferences on the structural style from seismic reflection data, regional tectonics and thermal-mechanical models of brittle-ductile deformation: Journal of Geophysical Research, v. 89, p. $5,733-5,762$.

Smith, R.B., Meertens, C.M., Lowry, A.R., Palmer, Randy, and Ribe, N.M., 1997, Evolution and active processes of the Yellowstone hotspot: Eos, Transactions of the American Geophysical Union, v. 78, no. 46, p. F801.

Sturchio, N.C., Pierce, K.L., Murrell, M.T., and Sorey, M.L., 1994, Uranium-series ages of travertines and timing of the last glaciation in northern Yellowstone area, Wyoming-Montana: Quaternary Research, v. 41, p. 265-277.

Tiller, C.C., 1995, Postglacial sediment stratigraphy of large lakes in Greater Yellowstone-Scenarios of tectonic and climatic forcing: Minneapolis, University of Minnesota, M.S. thesis, $193 \mathrm{p}$.

Vasco, D.W., Smith, R.B., and Taylor, C.L., 1990, Inversion for sources of crustal deformation and gravity change at the Yellowstone caldera: Journal of Geophysical Research, v. 95, no. B12, p. 19,839-19,856.

Waddington, J.C.B., and Wright, H.E., Jr., 1974, Late Quaternary vegetation changes on the east side of Yellowstone Park, Wyoming: Quaternary Research, v. 4, p. 175-184.

Waite, G.P., and Smith, R.L., 2002, Seismic evidence for fluid migration accompanying subsidence of the Yellowstone caldera: Journal of Geophysical Research, v. 107, no. B9, 2177, doi:10.1029/2001JB000586. 
Wheeler, R.P., 1954, Two new projectile point types-Duncan and Hanna Points: Plains Anthropologist, no. 1, p. 7-14.

Whipple, J.J., 2001, Yellowstone sand verbena-A Yellowstone Lake endemic [abs.], in Yellowstone Lake, hotbed or chaos or reservoir of resilience?: 6th Biennial Scientific Conference on the Greater Yellowstone Ecosystem, Yellowstone National Park, Wyoming, Oct. 8-10, 2001, p. 17.
Whitlock, Cathy, 1993, Postglacial vegetation and climate of Grand Teton and southern Yellowstone National Parks: Ecological Monographs, v. 63, no. 2, p. 173-198.

Wicks, Charles, Jr., Thatcher, Wayne, and Dzurisin, Daniel, 1998, Migration of fluids beneath Yellowstone caldera inferred from satellite radar interferometry: Science, v. 282, p. 458-462.

Yokoyama, I., 1971, Pozzuoli event in 1970: Nature, v. 229, p. 532-534. 